University of Louisville

ThinkIR: The University of Louisville's Institutional Repository

8-2006

\title{
The application of Maxwell elements for modeling, identification and analysis of passive and semi-active vibration systems.
}

Jie Zhang 1959-

University of Louisville

Follow this and additional works at: https://ir.library.louisville.edu/etd

\section{Recommended Citation}

Zhang, Jie 1959-, "The application of Maxwell elements for modeling, identification and analysis of passive and semi-active vibration systems." (2006). Electronic Theses and Dissertations. Paper 1637. https://doi.org/10.18297/etd/1637

This Doctoral Dissertation is brought to you for free and open access by ThinkIR: The University of Louisville's Institutional Repository. It has been accepted for inclusion in Electronic Theses and Dissertations by an authorized administrator of ThinkIR: The University of Louisville's Institutional Repository. This title appears here courtesy of the author, who has retained all other copyrights. For more information, please contact thinkir@louisville.edu. 
THE APPLICATION OF MAXWELL ELEMENTS FOR MODELING, IDENTIFICATION AND ANALYSIS OF PASSIVE AND SEMI-ACTIVE VIBRATION SYSTEMS

By

Jie Zhang

B.S., Zhejiang University, Hangzhou, China, 1982

M.S., Zhejiang University, Hangzhou, China, 1989

\author{
A Dissertation \\ Submitted to the Faculty of the \\ Graduate School of the University of Louisville \\ in Partial Fulfillment of the Requirements \\ for the Degree of
}

Doctor of Philosophy

Department of Mechanical Engincering

University of Louisville

Louisville, Kentucky

August 2006 
THE APPLICATION OF MAXWELL ELEMENT FOR MODELING, IDENTIFICATION AND ANALYSIS OF PASSIVE AND SEMI-ACTIVE VIBRATION SYSTEMS

\author{
By
}

Jie Zhang

B.S., Zhejiang University, Hangzhou, China, 1982

M.S., Zhejiang University, Hangzhou, China, 1989

A Dissertation Approved on

June 30, 2006

by the following Dissertation Committee:

Dissertation Director 


\section{DEDICATION}

To

\section{My wife Zhongwei Qi, \\ My son Chengyi Zhang}

$\&$

My parents

Mr. Yongfang Zhang and Ms. Yan Gu,

Who have given me invaluable support

I dedicate this work with love and gratitude 


\section{ACKNOWLEDGMENTS}

I sincerely thank and gratefully acknowledge my great mentor Dr. Christopher M. Richards, for his intellectual and continued support and guidance throughout cvery step of my dissertation studies. His mentoring and friendship mean a lot to me. I greatly admire his professionalism, dedication and kindness and I am grateful for everything that he taught me.

I sincerely thank my committee members, Drs. G. Prater, J. P. Wong, W. P. Hnat and T. A. Weigel for their helpful suggestions during my research work and thorough review of the dissertation. I would also like to acknowledge the many extraordinary pcople in the Mcchanical Department, both professors and students, whom I had the chance to meet, work with and learn from during my graduate studies. Many thanks to my friends and colleagues who provide a very pleasant and work-motivated atmosphere. Special thanks to Mr. J. J. Jones for his kind and help to my experimental works.

Last but not least. I would like to express my heartfelt gratitude to my wife for her continued encouragement, support and caring on my studies. I would also to express my heartfelt gratitude to my son. Although you may not really understand what Dad's studies mean, you are always the biggest motivation of Dad's work. I am in debeted to my parents for the foundations that they have given me while growing up and to my sister 
for her continued kinchess and caring. I have been blessed with a loving and supporting family and I an deeply grateful for that. 


\author{
ABSTRACT \\ THE APPLICATION OF MAXWELL ELEMENT FOR MODELING, \\ IDENTIFICATION AND ANALYSIS OF \\ PASSIVE AND SEMI-ACTIVE VIBRATION SYSTEMS
}

Jie Zhang

June 23, 2006

Dynamic analysis and parameter identification of a single mass elastomeric isolation system represented by Maxwell model is examined using both analytical and experimental approaches in this dissertation. Influences that the stiffness and damping values of the Maxwell element have on natural frequency, damping ratio and frequency response are uncovered and three unique categories of Maxwell-type elements are defined. It is revealed through analytical examples that Maxwell models consisting of two Maxwell elements can accurately replicate the dynamic behavior of Maxwell systems having two or more Maxwell elements. Two parameter identification methods are developed for identifying Maxwell models from measured frequency response spectra. To experimentally evaluate the analytic results, three different commercial rubber mounts are considered. For all three rubber isolators, it is shown that Maxwell models with two Maxwell elements can accurately represent the measured static and dynamic characteristics of the real elastomeric isolation systems. 
Aeroelastic aircraft wings are the structures which have variable natural frequency and damping ratio as flight parameters change. Serious vibration inhibits the flight at high airspeed conditions. In this study, the dynamic analysis of acroelastic aircraft wings reveals that a DVA (dynamic vibration absorber) with tunable stiffness and damping parameters can effectively suppress vibration over variable airspeeds in the presence of broadband external disturbance. Since tunable stiffness components are not yet well developed, another configuration of a semi-active DVA having only one tunable damping component is designed. Dynamic analysis reveals that the performance of this semiactive DVA is very close to the DVA having both tunable stiffness and damping components. Two control methods are developed for the semi-active DVA. The first control method is based on the measured airspeed. It works well if the air density is constant during the flight. The second method, a neural-network based controller, is formulated directly in terms of ready measured normalized vibration response spectra. It works well with time-varying airspeed and air density. Both methods are based on measured data and do not require prior knowledge of the plant mathematic model. 


\section{TABLE OF CONTENTS}

PAGE

ACKNOWLEDGMENTS $\mathrm{v}$

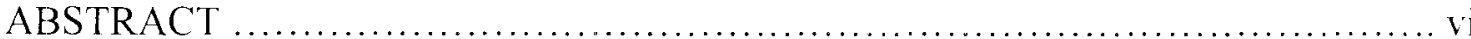

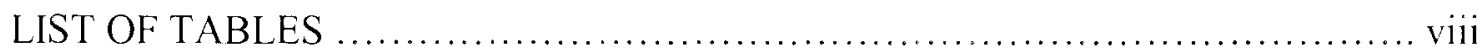

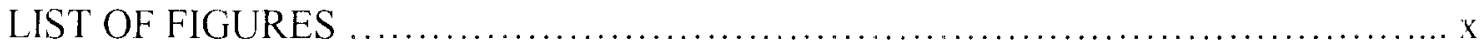

\section{CHAPTER}

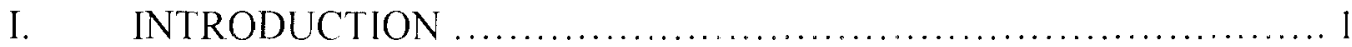

1.1. Modeling Methods of Elastomeric Isolators ..................... 2

1.2. Tunable Dynamic Vibration Absorbers ............................ is

II. LITERATURE REVIEW ..................................... 9

2.1. Modeling, Analysis and Identification of Passive

Vibration Isolators ............................................... 9

2.2. Application and Control of Tunable Dynamic

Vibration Absorbers ................................................. 11

III. DYNAMIC ANALYSIS AND PARAMETER IDENTIFICATION

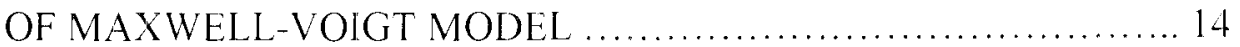

3.1. Problem Formulation ....................................... 14

3.2. Modal Analysis .......................................... 1?

3.2.1. The $\ln f$ luences of $k l$ and $\mathrm{cl}$ on natural frequency ........ 1 ?

3.2.2. The Influences of $\mathrm{kl}$ and $\mathrm{cl}$ on damping ratio ...........21 
3.3. Harmonic Analysis ............................................ 25

3.4. Parameter Identification .................................... 29

3.5. Summary ..................................................... 40

IV. DYNAMIC ANALYSIS AND PARAMETER IDENTIFICATION

OF GENERAL MAXWELL MODEL $\ldots \ldots \ldots \ldots \ldots \ldots \ldots \ldots \ldots \ldots . \ldots . \ldots \ldots$

4.1. Problem Formulation ....................................... 42

4.2. Identification of General Maxwell Systems Using M-V Models .... 43

4.3. Parameter Identification Using Constraint Optimization ........... 50

4.3.1. Parameter identification of $M-V$ systems ................ 50

4.3.2. Parameter identification of $M-M-V$ systems ............. 54

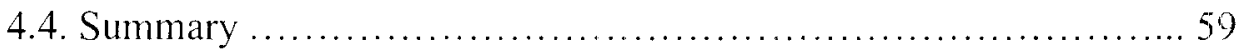

V. PARAMETER IDENTIFICATION OF RUBBER ISOLATORS ........61

5.1. Static Stiffness Experimental Setup and Results ................... 61

5.2. Frequency Response Experimental Setup and Results ............. 64

5.3. Parameter Identification ................................... 67

5.3.1. Voigt model parameter identification ................... 6?

5.3.2. Parameter identification as Maxwell-Voigt model ........ 70

5.3.3. Parameter identification of $M-M-V$ models by

constrain optimization .................................... 74

5.4. Summary .............................................. 78

VI. DYNAMIC ANALYSIS OF AN AEROELASTIC AIRCRAFT WING WITH DYNAMIC VIBRATION ABSORBER …................... 79

6.1. Dynamic Analysis of an Aeroclastic Aircraft Wing ............... 79 
6.1.1. Problem formulation 79

6.1.2. Modal analysis 81

6.1.3. Impulse response analysis 82

6.1.4. Simulation results 85

6.2. Dynamic Analysis of an Aeroelastic Aircraft Wing with DVA 86

6.2.1. Problem formulation 86

6.2.2. Optimized parameters of DVA 88

6.2.3. Comparison with the performance of constant parameter DVA 99

6.2.4. Simulation results 101

6.3. Tunable DVA Design and Dynamic Analysis 105

6.3.1. Tunable DVA design and problem formulation 105

6.3.2. Optimized parameters of tunable Maxwell

element DVA 108

6.3.3. Simulation results 114

6.4. Summary 116

VII. SEMI-ACTIVE VIBRATION CONTROL 118

7.1. The Control Method based on Airspeed

7.1.1. Objective of control

7.1.2. Flight Simulation

7.1.3. Simulation with variable air density

7.2. Control by Vibration Spectra Signals

7.2.1. System identification 
7.3. Summary 136

VIII. CONCLUSIONS AND DIRECTIONS FOR TUTURE RESEARCH .. 138

8.1. Research on Elastomeic Isolators 138

8.2. Research on DVA Control ................................... 141

REFERENCES 143 


\section{LIST OF TABLES}

TABLE

PAGE

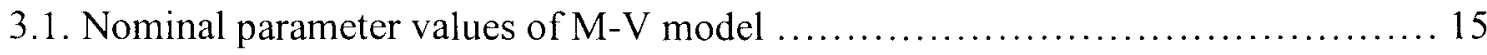

3.2. Parameters of three Voigt models .......................................... 26

3.3. Parameters of "Experimental" M-V systems ................................. 30

3.4. Parameters of identified Voigt models ........................................ 30

3.5. Parameters of M-V Model A for M-V System A ................................ 35

3.6. Parameters of M-V Model B for M-V System B ............................... 37

3.7. Parameters of M-V Model C for M-V System C .............................. 39

4.1. Parameters of actual M-M-V Systems ...................................... 46

4.2. Parameters of Identified M-V Models ....................................... 47

4.3. Identified parameters of $\mathrm{M}-\mathrm{V}$ models using optimization method .................. 51

4.4. Parameters of Identified M-M-V Model F ................................... 54

4.5. Parameters of general Maxwell systems …................................. 57

4.6. Parameters of identified general Maxwell models .............................. 58

5.1. Static stiffness of rubble mounts ............................................ 64

5.2. Natural frequency and damping ratio of rubble mounts ....................... 67

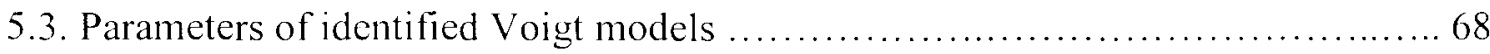

5.4. Parameters of identified Maxwell-Voigt models (Trial 1) ....................... 71 
5.6. Parameters of identified general Maxwell models ................................ 76

6.1. Critical airspeed velocity with different DVA position .......................... 94

6.2. Critical airspeed velocity with different DVA mass ........................... 96

6.3. Critical airspeed velocity with different constant DVA ......................... 99 


\section{LIST OF FIGURES}

FIGURE

PAGE

1.1. Analytical model of elastomeric isolator represented by

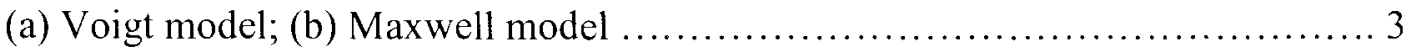

1.2. Frequency response spectra of Voigt model and Maxwell-Voigt model ............. 4

3.1. Definition of Maxwell element type ...................................... 16

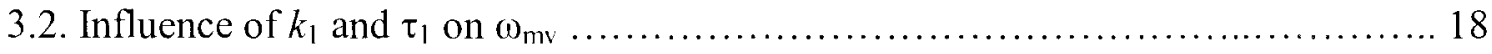

3.3. Influence of $k_{1}$ and $\tau_{1}$ on $\omega_{m}$ for a model with $k=200 \mathrm{kN} / \mathrm{m}$

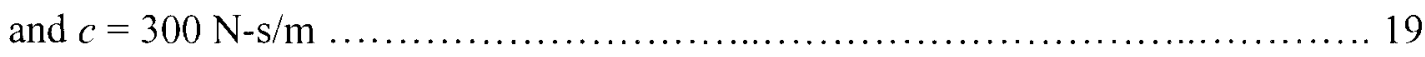

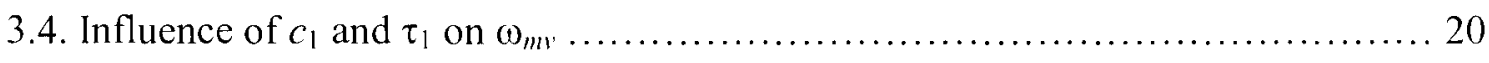

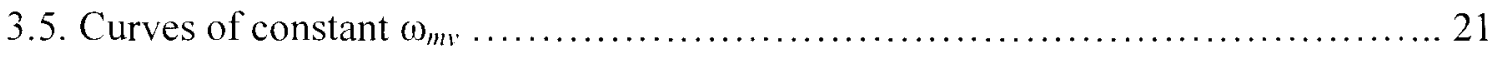

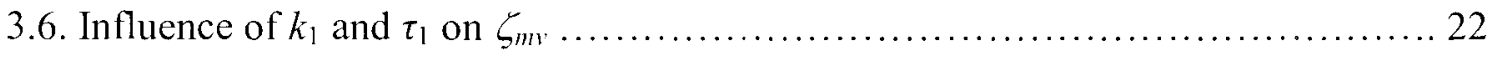

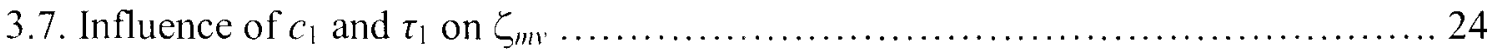

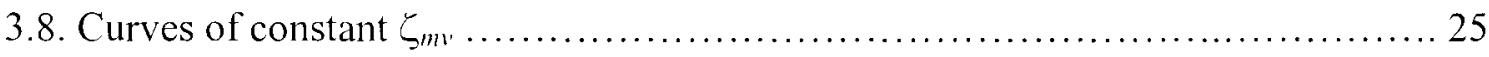

3.9. Frequency response spectra of three Voigt models ............................ 26

3.10. The influence of $\tau_{1}\left(c_{1}\right.$ is constant) on $M-V$ frequency response model $\ldots \ldots \ldots \ldots 27$

3.11. The influence of $\tau_{1}\left(k_{1}\right.$ is constant) on M-V frequency response model .......... 28

3.12. Frequency response spectra of Voigt Models $M$ and $K$ and "experimental"

$\mathrm{M}-\mathrm{V}$ systems with equivalent natural frequencies and damping ratios 
3.13. Identifying $k_{1}$ and $c_{1}$ from constant $\omega_{m}$ and $\zeta_{m}$ curves

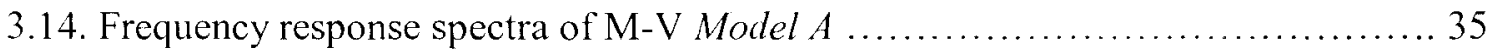

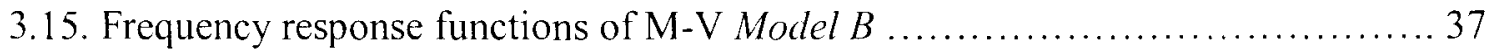

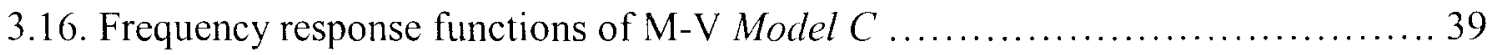

4.1. Mathematic model of isolator represented by general Maxwell model ........... 42

4.2. Frequency response of M-M-V Systems and corresponding identified M-V models (a) System and Model $A$; (b) System

and Model B; (c) System and Model C; (d) System and Model D;

(e) System and Model E; (f) System and Model F ........................... 48

4.3. Frequency response functions of (a) M-V System A and M-V

Model A $(\sigma=0.05)$; (b) M-V System B and M-V Model B

$(\sigma=0.05) ;(\mathrm{c}) \mathrm{M}-\mathrm{V}$ System $C$ and M-V Model $C(\sigma=0.05)$

4.4. Frequency response spectra of actual M-M-V System $F$ and

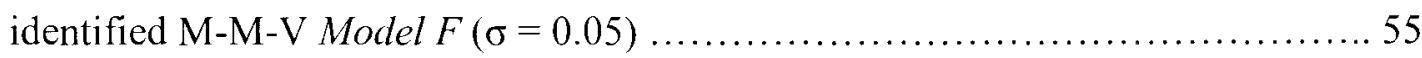

4.5. The frequency response spectra of (a) general Maxwell System A and identified M-M-V Model A; (b) general Maxwell System B and identified M-M-V Model B 59

5.1. (a) Bubble mount; (b) plate mount; (c) rubber stud ........................ 61

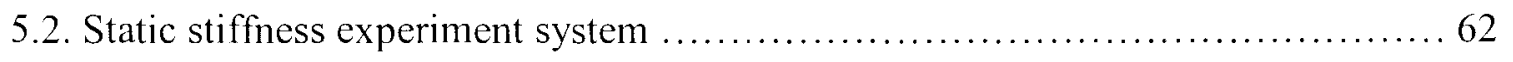

5.3. Axial loads vs. deflections of (a) bubble mount; (b) plate mount;

(c) rubber stud 63

5.4 Dynamic experiment system 64

5.5. Frequency response spectra of (a) bubble mount; (b) plate mount; 
(c) rubber stud 66

5.6. Frequency response spectra of (a) bubble mount; (b) plate mount;

(c) rubber stud

5.7. Frequency response spectra of (a) bubble mount; (b) plate mount;

(c) rubber stud (Trial 1) 72

5.8. Frequency response spectra of (a) bubble mount; (b) plate mount;

(c) rubber stud (Trial 2) 73

5.9. Frequency response spectra of (a) bubble mount; (b) plate mount;

(c) rubber stud 77

6.1. Analytical model of pitch-plunge airfoil 79

6.2. Natural frequencies and damping ratios v.s. airspeed velocity 82

6.3. Impulse time responses of pitch-plunge airfoil 84

6.4. Flight simulation of aeroelastic aircraft wing 85

6.5. Analytical model of pitch-plunge airfoil with absorber $\ldots \ldots \ldots \ldots \ldots \ldots \ldots \ldots \ldots$

6.6. Minimum damping ratios vs. DVA parameters when airspeed is $400 \mathrm{~m} / \mathrm{s} \ldots \ldots . .90$

6.7. Minimum damping ratios vs. DVA parameters when airspeed is $800 \mathrm{~m} / \mathrm{s} \ldots \ldots \ldots 91$

6.8. Minimum damping ratios vs. DVA parameters when airspeed is $850 \mathrm{~m} / \mathrm{s}$ 92

6.9. Minimum damping ratios vs. DVA parameters when airspeed is $900 \mathrm{~m} / \mathrm{s}$ 93

6.10. Optimized Minimum damping ratios where distance is (a) $0.27 \mathrm{~m}$;

(b) $0.15 \mathrm{~m} ;$ (c) $-0.8 \mathrm{~m} ;$ (d) $-1.53 \mathrm{~m}$ 95

6.11. Optimized Minimum damping ratios where mass is (a) $10 \mathrm{~kg}$;

(b) $20 \mathrm{~kg}$; (c) $30 \mathrm{~kg}$; (d) $40 \mathrm{~kg}$ 97

6.12. Optimized values of DVA parameters $c_{1}$ and $k_{1}$ 98 
6.13. Optimized Minimum damping ratios of constant DVA

6.14. Time responses of pitch-plunge airfoil with constant DVA(1) vs. airspeed velocity

6.15. Time responses of pitch-plunge airfoil with constant DVA(2) vs. airspeed velocity 103

6.16. Time responses of pitch-plunge airfoil with constant DVA(3) vs. airspeed velocity 104

6.17. Time responses of pitch-plunge airfoil with tunable DVA vs. airspeed velocity 105

6.18. Analytical model of pitch-plunge airfoil with absorber 106

6.19. The variation of minimum damping ratios as $c_{2}$ changes 110

6.20. Optimized Minimum damping ratios and associated $c_{2}$ at different airspeed $\ldots . .110$

6.21. Natural frequencies and damping ratios of tunable Maxwell element DVA .... 111

6.22. The variation of minimum damping ratios as $c_{2}$ changes

6.23. Optimized Minimum damping ratios and associated $c_{2}$ at different airspeed $\ldots . .113$

6.24. Natural frequency and damping ratio of tunable Maxwell element DVA 113

6.25. Time responses of pitch-plunge airfoil with first tunable Maxwell element DVA 115

6.26. Time responses of pitch-plunge airfoil with second tunable Maxwell element DVA 116

7.1. Translational RMS of vibration responses changes as the values of $c_{2}$ changes at different airspeed changes

7.2. Rolational RMS of vibration responses changes as the 
values of $c_{2}$ changes at different airspeed changes

7.3. Weighted sum RMS of vibration responses changes as the

values of $c_{2}$ at different airspeed changes

7.4. Optimized damping coefficients and the RMS of vibration response 122

7.5. Time responses of pitch-plunge airfoil with tunable Maxwell element DVA

7.6. Time responses of pitch-plunge airfoil with tunable Maxwell element DVA with variable air density $\mu=0.05$

7.7. Time responses of pitch-plunge airfoil with tunable Maxwell element DVA with variable air density $\mu=0.1$ 126

7.8. Configuration of system identification process 128

7.9. Architecture of radial basis neuron 128

7.10. Architecture of radial basis Network 130

7.11. Comparison of the value of $c_{2}$ from neural network model and optimized value of $c_{2}$ from experiment simulation

7.12. Configuration of the control system with the neural-network based controller ... 132

7.13. Time responses of pitch-plunge airfoil with neural-network based controller .... 133

7.14. The values of $c_{2}$ during flight simulation 134

7.15. Time responses of pitch-plunge airfoil with the neural-network based controller with variable air density $\mu=0.05$ 135

7.16. Time responses of pitch-plunge airfoil with the neural-network based controller with variable air density $\mu=0.05$ 136 


\section{CHAPTER I}

\section{INTRODUCTION}

The requirement of reducing vibration of constructions and structures arises in a variety of engineering fields. There are numerous ways and means of preventing unacceptable vibrations. Most of them belong to two catalogs: vibration isolation and absorption. In most of the vibration isolation applications, the conventional passive rubber mounts provide an efficient way of reducing vibration transmission, but suffer from the problem of modeling the complicated dynamic properties of elastomeric materials. The modeling and parameter identification of passive isolators are very important for the application and design of isolation system. The DVA (dynamic vibration absorber) is a device for suppressing the vibration of constructions and structures. It consists of an additional mass which is connected by means of an elastic element to the structure needing protection. It is quite effective in reducing narrowband vibrations, but the application is limited in the cases of the natural frequencies of systems are variable and broadband external disturbance exists. Aeroelastic aircraft wings are the example structures which is not only subjected to disturbance forces with unpredictable waveform and a broadband spectrum, but also have a variable frequencies and damping ratios as airspeed and other variable flight parameters change. As airspeed increases to a critical airspeed - the airspeed at which one of the system's damping ratios become 
negative, damping ratio of aeroelastic aircraft wings can be negative, the system turns to be unstable and serious vibration occurs. That inhibits the flight of aircraft close or above the critical airspeed. For this application, tunable stiffness and damping properties are necessary for DVA to effectively suppress the vibration.

In this dissertation, the researches are focused on the modeling, analysis and parameter identification of elastomeric isolators and the design, analysis and control of semi-active DVA to suppress the vibration of the aeroelastic aircraft wings.

\subsection{Modeling Methods of Elastomeric Isolators}

The elastomeric isolator is widely used in noise and vibration control as means of vibration isolation components. For a successful prediction of dynamic behavior of an elastomeric isolation system which subjects given excitation, it is important to have a correct analytical model and accurate parameters of the isolator. Accurate modeling and parameter identification of rubber isolators is important for the dynamic analysis and design of mounting systems.

There are several approaches of using discrete springs and dashpots to build dynamic models of rubber isolators [1-4]. The Voigt model (Fig. 1.1(a)), which is a spring and damper in parallel, is often used for modeling elastomeric isolators owing to its simplicity in analysis and parameter identification [5]. Dynamic tests of a SDOF (single-degree-of-freedom) vibration system in which the rubber isolator is subjected to sinusoidal deformation are carried out to identify its dynamic properties. However, dynamic stiffness experiments often reveal the frequency dependent features of elastomeric isolators, can not be accurately simulated by the Voigt model $[4,6]$. The 
frequency-dependent complex stiffness model is an approach which allows the stiffness to be measured as a function of excitation frequency [5-7]. Although this model is useful for frequency domain analysis, it can be difficult to implement in the time domain [8]. Often, one or several Maxwell elements, which are the combination of a spring and a damper in series, are included in the model to simulate the dynamic behavior of elastomeric and other types of viscoelastic materials. This linear time domain model is known as Maxwell model (Fig. 1.1(b)). It is used when isolator dynamic stiffness is found to be frequency dependent and can simulate viscoelasticity properties in many different applications [3].
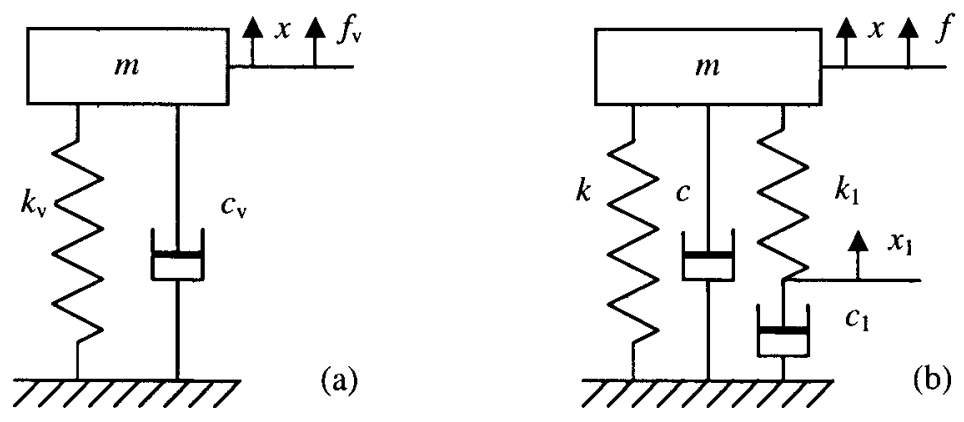

Fig. 1.1. Analytical model of elastomeric isolator represented by (a) Voigt model;

(b) Maxwell-Voigt model.

However, the influences Maxwell elements have on system dynamic properties (i.e., natural frequency, damping ratio and frequency response) are not yet discussed in detail; and, understanding these influences is important when attempting to identify a model with Maxwell elements that accurately represents a real elastomeric isolation system. For example, consider the two isolation models illustrated in Fig. 1.1 with the 
same isolation mass and whose stiffness and damping parameters are chosen to yield equivalent natural frequencies and damping ratios. Figure 1.2 illustrates that the frequency response spectra of the two models differ even though their masses, natural frequencies and damping ratios are equal. This illustration is important from a system identification standpoint since if the mass, natural frequency and damping ratio of an experimental single mass rubber isolated system are known, then the frequency response of a Voigt model chosen to represent this system is unique. However, since the MaxwellVoigt model, which has one Maxwell element and one Voigt element in model, possesses more parameters (i.e., $k_{1}$ and $c_{1}$ ) than the Voigt model, the frequency response function is not unique even though the natural frequency and damping ratio remain fixed. This enables more flexibility for simulating the dynamic behavior of the elastomeric isolation system over a broader frequency range.

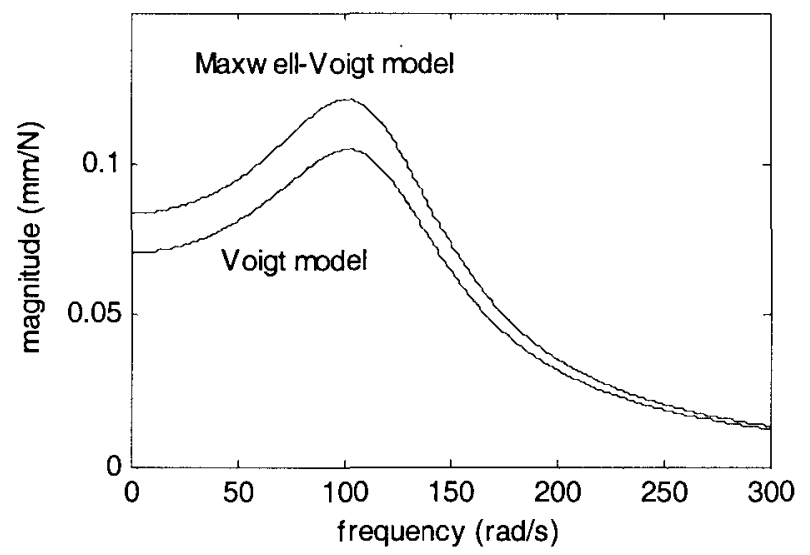

Fig. 1.2. Frequency response spectra of Voigt model and Maxwell-Voigt model.

In this dissertation, a parametric study is conducted to investigate the influences that stiffness and damping of a Maxwell element have on the natural frequency, damping 
ratio and frequency response of a single mass vibration isolation system as illustrated in Fig. 1.1(b). Modal analysis results reveal interdependent relations that $k_{1}$ and $c_{1}$ have on natural frequency and damping ratio. Also, three categories of Maxwell elements are defined, each with significantly different characteristics. It is also shown that Voigt and M-V (Maxwell-Voigt) models with equivalent natural frequencies and damping ratios can have considerably different frequency response functions.

Then, two parameter identification methods are developed for identifying M-V model and general Maxwell model (which has two or more Maxwell elements) from frequency response spectra. One method is based on constant natural frequency and damping ratio curves generated from modal analysis of potential M-V model. Another parameter identification method is suited for general Maxwell models having one or more Maxwell element by fitting the model to measured frequency response spectrum by means of constraint optimization. Studies conducted with analytical systems reveal that a Maxwell model having only one Maxwell element can simulate the dynamic characteristics of a Maxwell system having two Maxwell elements if they do not belong to a specific combination of Maxwell element Types. The effectiveness of both identification methods is verified by several numerical examples. However, further results show that the general Maxwell model with only one or two Maxwell elements can simulate the dynamic behavior of a general Maxwell system that has three or more Maxwell elements.

To exam the Maxwell model identification approach experimentally, three different rubber isolators are subjected to both static and dynamic excitations. It is shown that a Voigt model is incapable of accurately modeling the static and dynamic 
characteristics of these isolators. Likewise, Maxwell models having only one Maxwell element can be identified to have the same natural frequency, damping ratio, static stiffness, of the isolators in a single mass configuration, although the frequency response spectra of these models do not match the measured frequency response spectra well. By using the approach of constraint optimization, the Maxwell models having two Maxwell elements are identified that have the same natural frequency, damping ratio and static stiffness of the isolators in a single mass configuration, while accurately replicating their measured frequency response spectra.

\subsection{Tunable Dynamic Vibration Absorbers}

Aeroelastic aircraft wings are the structures which are subjected to disturbing forces with unpredictable waveform and a broadband spectrum. The dynamic properties, such as natural frequencies and damping ratios, vary during flight as airspeed and other flight parameters change. As airspeed increases to the critical airspeed, the minimum damping ratio is negative and the system turns to be unstable. Serious vibration occurs that inhibits the flight at high airspeed. The DVA is a very useful device that is used to suppress vibrations [9] and even minimizes sound radiation [10]. The beneficial effects of a typical DVA are obvious over a very narrow frequency range. It induces two resonance peaks away from the design frequency, which may cause problems in some cases. The viscoelastic materials which exhibit stiffness and damping properties vary nonlinearly with excitation frequency are well suited for efficient vibration control over a wide frequency range [11]. Tunable DVAs are capable of changing their mass, stiffness, or damping properties. In case of the DVA's stiffness is tunable, its natural frequency 
can be changed depending on its working condition [12]. It is suited to broad-band vibration control.

In this dissertation, the feasibility of tunable DVA application for aeroelastic aircraft wings vibration suppression is investigated. The DVA with tunable stiffness and damping properties can behave much better than the DVA which has constant stiffness and damping properties. Because tunable stiffness component is not well developed yet, a new configuration of DVA which has a tunable damper in series with a normal spring is designed and is called tunable Maxwell element DVA in this dissertation. Adjusting the parameter of the variable damper can change the damping and stiffness properties of tunable Maxwell element DVA at the same time. The behavior of this tunable Maxwell element DVA is very close to the behavior of the DVA which has both variable damper and stiffness components. Because the tunable damper, such as electrorheological or magnetorheological damper, is well developed and has been used in variety of engineering applications, using tunable Maxwell element DVA has a big advantage over using tunable DVA which has a tunable stiffness component included.

Two control algorithms for the tunable Maxwell element DVA are developed. Because the natural frequency and damping ratios of aeroelastic aircraft wings change as airspeed changes, the first algorithm is based on the measured airspeed. The relationship between measured airspeed and optimized damping parameter of the tunable damper is found by minimizing the RMS (root means square) of system vibration responses. The second algorithm is based on the spectra of system vibration responses. The relationship between the spectra of system vibration response and optimized damping parameter is established by a neural networks model. This algorithm is good in the cases where both 
airspeed and air density are variable. Both algorithms are verified by numerical simulation. 


\section{CHAPTER 2}

\section{LITERATURE REVIEW}

\subsection{Modeling, Analysis and Identification of Passive Vibration Isolators}

To accurately model rubber isolators, the correct analytical model and parameters need to be identified. Dynamic stiffness experiments are often carried out for this purpose, where high frequency excitation reveals the frequency dependent features [4]. In addition, the dynamic stiffness of rubber isolators can be found to vary with amplitude of displacement, frequency of excitation, and pre-compression [7-8, 14-16]. To address these issues, new experimental and analytical methods have been developed. An indirect experimental method has been developed for extracting the dynamic properties in the high frequency range where translational and rotational dynamic stiffness are also measured [7]. Another experimental identification method has been developed for determining frequency dependent multi-dimensional dynamic stiffness, where a multidimensional mobility synthesis formulation is utilized for describing and extracting dynamic properties of rubber isolators [8]. The influence of pre-compression on the dynamic properties of isolators has been investigated [14-15]. The proposed dynamic model is based on the relationship between the phase velocity and compression ratio of the rubber isolator. The dynamic stiffness of a cylindrical isolator has been investigated via experiment and was found to be strongly dependent on pre-compression in the high 
frequency range [16]. Vibration transmission through isolators has also been investigated in multi-degree-of-freedom (MDOF) configuration as another measure of rubber isolator performance [17]. Alternatively, the continuous system theory has been used for modeling the vibration transmission through rubber isolators where the flexural and longitudinal motions are considered simultaneously [18]. The finite element method has been used for static simulation of rubber isolators [19]. Isolators under large deformation represented by axisymmetric, quarter-symmetric and three dimensional finite element models were meshed and deformation was calculated using commercially available finite element software. Predicted static properties were shown to match well with experimental results.

To address the nonlinear viscoelastic behavior of rubber isolators, nonlinear models are often identified and utilized [20-25]. The nonlinearities were often described with cubic nonlinear terms [20]. A Coulomb damper was also included for the purpose of improving the performance of nonlinear rubber isolators [21]. The existence of a Coulomb damper was shown to improve the performance when the shock displacement of the base movement decays quickly. The method of estimating cubic spring parameters under random excitations has been developed [22]. The discussion includes the roles of cubic hardening springs, cubic softening springs, and tangent springs. The investigation of linear and nonlinear transient performance of engine mounts has also been conducted both analytical and experimentally and a model for predicting the response to transient events was developed [23]. The identification of nonlinear isolators using temporal and spectral methods has been investigated [24]. It was found that non-integer exponent-type terms were best for describing the nonlinear elastic force of the rubber. More systematic 
experiments were also conducted in both SDOF and MDOF configurations while static, random, frequency-sweep, and fixed frequency excitations were applied where the nonlinear dynamic behavior of three different isolators was clarified [25]. Due to the versatility, Bouc-Wen differential models are often used for representing friction-type hysteretic isolators [26]. A parameter identification method based on least squares estimation in the frequency domain is developed for the Bouc-Wen model using experimental data from periodic vibration experiments. A bilinear hysteretic model is an alternative method for hysteretic isolators [27]. The parameters are identified by minimizing the discrepancy between the measured responses and the theoretical responses of the system in the time domain. The equivalent linearization technique has also been developed to linearize the governing nonlinear multi-degree-of-freedom equations of motion where responses of interest are calculated from the linearized governing equations of motion [28]. Nonetheless, it is often a challenge to determine a unique model and parameter identification process that yields an accurate model for dynamic analysis of elastomeric isolators for many different loading conditions.

Although the aforementioned studies utilize many successful modeling, analysis and parameter identification methods for elastomeric isolators, it is often important to include Maxwell elements in the models when it is known that frequency dependent damping and stiffness properties exist [3].

\subsection{Application and Control of Tunable Dynamic Vibration Absorbers}

Increasing research efforts are being spent on the development of tunable DVA in the past decades. The tunable DVA using adjustable pneumatic springs can achieve 
significantly transmissibility reduction than a conventional isolator over a limited frequency band [29]. The frequency at which maximum vibration supression occurs can be changed at real time over $12 \mathrm{~Hz}$ frequency band with experimental device. A tuning mass damper method is proposed and used for tunable DVA application [30]. The static output feedback active controller is designed via an optimization approach. It is also applicable to multi-input systems. The performance of DVA which has two-DOF is investigated [31]. It was found that the two-DOF DVA achieves better performance than the optimized SDOF DVA, and even better than two separate optimized DVA.

A tunable piezoelectric absorber and an active tuning method were developed for effective stiffness adjusting electrically [32]. A control scheme was developed to estimate the desired tuning frequency from experiment signals. The tuning range is bounded by its short- and open-circuit resonance frequencies. The design issues related to the vibration absorbers using single-crystals piezoceramics were identified and addressed [33]. A wide tuning range and structural application are achievable, as opposed to similar devices which employ polycrystalling piezoceramics. Because the piezoceramics materials possess nonlinear characteristics, an equivalent linear model is usually developed for the absorber subsystem [34]. The approximate parameters of actuators would result in partial vibration suppression when utilized in real applications. An auto-tuning method is used to effectively tune the parameters of actuator to improve the vibration suppression quality.

The feasibility of DVA with a sliding mass which works as a friction damper is investigated [35]. The resonance peaks may be reduced substantially by the effect of the friction damper and the damper can be tuned for providing damping in a broad range of 
frequency. A proof-of-concept "smart spring" hardware model has been designed, analyzed, built, and tested for helicopter individual blade control [36]. Both mathematical and experimental results have showed positive support for the viability of using "smart spring" blade vibration control. Although the "smart spring" is used in an isolator, it is worth to investigate using it in DVA. Stiffness of shape memory alloy spring element can be changed through heating and results in changing the natural frequency of DVA [37]. The relationship between the shape memory alloy beam element temperature and the stiffness of absorber were developed. Testing results showed that the natural frequency of absorber could be varied by approximately $15 \%$, with a corresponding reduction in the steady state vibration of the primary system of up to $40 \mathrm{~dB}$.

An automatic tuning algorithm based on online parameter identification is developed for increasing robustness against uncertainties and fluctuations in the properties of the DVA subsystems [38]. The control algorithm is accomplished in a single step which avoids the convergence concerns and reduces the time requirement. Four control methods for semi-active tuned vibration absorbers are compared [39]. The parameters of absorbers are optimized based on minimization of peak transmissibility. The research results show that all of the semi-active vibration absorbers perform better than passive model and the on-off groundhook control performs the best among the considered methods. The robustness analysis is offered because off-tuned vibration absorbers may amplify the vibration of the primary structures [40]. The test apparatus was built with magneto-rheological damper. The experimental results show that the semi-active vibration absorber is more robust than passive vibration absorbers. 


\section{CHAPTER 3}

\section{DYNAMIC ANALYSIS AND PARAMETER IDENTIFICATION OF \\ MAXWELL-VOIGT MODEL}

\subsection{Problem Formulation}

The governing equations of motion for the $\mathrm{M}-\mathrm{V}$ model represented in Fig. 1.1(b) are,

$$
\left[\begin{array}{cc}
m & 0 \\
0 & 0
\end{array}\right]\left\{\begin{array}{c}
\ddot{x} \\
\ddot{x}_{1}
\end{array}\right\}+\left[\begin{array}{cc}
c & 0 \\
0 & c_{1}
\end{array}\right]\left\{\begin{array}{l}
\dot{x} \\
\dot{x}_{1}
\end{array}\right\}+\left[\begin{array}{cc}
k+k_{1} & -k_{1} \\
-k_{1} & k_{1}
\end{array}\right]\left\{\begin{array}{l}
x \\
x_{1}
\end{array}\right\}=\left\{\begin{array}{l}
f \\
0
\end{array}\right\}
$$

Writing Eq. (3.1) in state-space form,

$$
\left\{\begin{array}{l}
\ddot{x} \\
\dot{x} \\
\dot{x}_{1}
\end{array}\right\}=\left[\begin{array}{ccc}
-\frac{c}{m} & -\frac{k+k_{1}}{m} & \frac{k_{1}}{m} \\
1 & 0 & 0 \\
0 & \frac{1}{\tau_{1}} & -\frac{1}{\tau_{1}}
\end{array}\right]\left\{\begin{array}{l}
\dot{x} \\
x \\
x_{1}
\end{array}\right\}+\left\{\begin{array}{l}
f^{\prime} \\
0 \\
0
\end{array}\right\}
$$

where $f^{\prime}=f / m$ and $\tau_{1}=c_{1} / k_{1}$ is the time constant of the Maxwell element. The nominal values used for the parameters of the M-V model are listed in Table 3.1 and unless specified otherwise, they will maintain these values throughout Chapter 3 . The modal analysis is based on the matrix in Eq. (3.2). The resulting undamped natural frequency $\omega_{m v}$ is $119.03 \mathrm{rad} / \mathrm{s}$ and the damping ratio $\zeta_{m v}$ is 0.36 . In the absence of the Maxwell element, the system reduces to a Voigt model,

$$
m \ddot{x}+c_{v} \dot{x}+k_{v} x=f_{v}, \quad \ddot{x}+2 \zeta_{v} \omega_{v} \dot{x}+\omega_{v}^{2} x=f_{v}^{\prime}
$$


where the natural frequency $\omega_{\nu}$ is $109.54 \mathrm{rad} / \mathrm{s}$ and the damping ratio $\zeta_{\nu}$ is 0.18 .

Table 3.1. Nominal parameter values of M-V model.

\begin{tabular}{|c|c|c|c|c|}
\hline$m(\mathrm{~kg})$ & $k(\mathrm{kN} / \mathrm{m})$ & $c(\mathrm{~N}-\mathrm{s} / \mathrm{m})$ & $k_{1}(\mathrm{kN} / \mathrm{m})$ & $c_{1}(\mathrm{~N}-\mathrm{s} / \mathrm{m})$ \\
\hline 1 & 12 & 40 & 12 & 40 \\
\hline
\end{tabular}

As a preliminary observation, notice that for the $\mathrm{M}-\mathrm{V}$ model, $c=c_{1}$, and $\zeta_{m v}$ is twice $\zeta_{v}$. Also $k=k_{1}$, however $\omega_{m v}$ is only slightly higher than $\omega_{v}$. It appears that the influence of $k_{1}$ on $\omega_{m v}$ is much less than the influence of $c_{1}$ on $\zeta_{m v}$; and, for this particular choice of parameters, $\omega_{m v}$ depends mainly on $k$ while $\zeta_{m v}$ depends equally on $c$ and $c_{1}$. This observation raises an important question when tuning an M-V model's natural frequency and damping ratio to match that of a real rubber isolated single mass dynamic system: how do the modal parameters of the model change to variations in the Maxwell element parameters? As will be shown, interdependence exists between $k_{1}$ and $c_{1}$ that alters how much the Maxwell parameters influence $\omega_{m v}$ and $\zeta_{m v}$. A second question also arises when modeling an experimentally measured frequency response spectrum of a rubber isolated single mass dynamic system is found to be inadequate using a conventional Voigt model: can an $\mathrm{M}-\mathrm{V}$ model provide a better fit? The chief objective of this study is to investigate these questions, i.e., how the parameters of the Maxwell element $\left(c_{1}\right.$ and $\left.k_{1}\right)$ influence $\omega_{m v}, \zeta_{m v}$, and frequency response of the single mass dynamic system.

For different ranges of values for $\tau_{1}$, three unique types of Maxwell elements are defined in this article, as depicted in Fig. 3.1. In general, for small values of $\tau_{1}$ the 
Maxwell element behaves like a pure damping element - the spring stiffness is so great that motion only occurs across the damper. As $\tau_{1}$ increases (i.e., the ratio of damping-tostiffness increases), some motion across the spring occurs, although damping still dominates. In this range, the element will be referred to as Type A. As $\tau_{1}$ continues to increase, a range exists in which the element is neither damping nor stiffness dominant. This will be referred to as a Type B element. Next, a range exists (Type $C$ ) in which the damping-to-stiffness ratio increases to a point in which motion is primarily across the spring and therefore the element becomes stiffness dominant. Finally, for large values of $\tau_{1}$, the element behaves like a pure spring - the damping is so great that motion only occurs across the spring. Note, the shading in Fig. 3.1 implies that there are no precise values of $\tau_{1}$ where the Maxwell element changes from Type A to Type $B$ or from Type $B$ to Type $C$. Instead, Type $A$ defines a transition range between pure damping characteristics and stiffness-damping-in-series characteristics, while Type $C$ defines a transition range between stiffness-damping-in-series characteristics and pure stiffness characteristics of the element. Also, these ranges are not universal, but instead depend on the parameters of the Voigt and Maxwell elements, i.e., $c, k, c_{1}, k_{1}$.

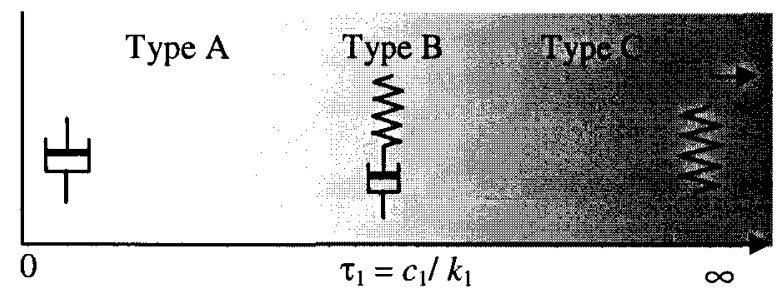

Fig. 3.1. Definition of Maxwell element type. 


\subsection{Modal Analysis}

The modal analysis is based on the eigenvalue problem of the system matrix in Eq. (3.2), which yields one real and one complex conjugate pair of eigenvalues and corresponding eigenvectors in the case of $\zeta_{\mathrm{mv}}<1$. For the purpose of single mass vibration system analysis, only the conjugated eigenvalues are of concern from which $\omega_{m v}$ and $\zeta_{m v}$ are determined.

The influences of $k_{1}$ and $c_{1}$ on $\omega_{m v}$ and $\zeta_{m v}$ will be illustrated in two ways. First, a family of constant $k_{1}$ curves will be graphed illustrating changes in $\omega_{m v}$ and $\zeta_{m v}$ as a function of $\tau_{1}$. Note that since $m, k, c$ and $k_{1}$ are held constant, changes in $\tau_{1}$ are proportional to changes in $c_{1}$. Next, a family of constant $c_{1}$ curves will be graphed illustrating changes in $\omega_{\mathrm{mv}}$ and $\zeta_{\mathrm{mv}}$ as a function of $\tau_{1}$. Here, since $m, k, c$ and $c_{1}$ are held constant, changes in $\tau_{1}$ are inversely proportional to changes in $k_{1}$.

\subsubsection{The Influences of $k_{1}$ and $c_{1}$ on natural frequency}

Figure 3.2 illustrates constant $k_{1}$ curves whereby changes in $\tau_{1}$ are proportional to changes in $c_{1}$ only. From these curves, three basic conclusions can be drawn. First, the presence of the Maxwell element always results in the natural frequency of the M-V model $\omega_{m v}$ greater than the natural frequency of the Voigt model $\omega_{v}$ regardless of the values for $k_{1}$ and $\tau_{1}$. Second, elements with larger values of $k_{1}$ have more influence on $\omega_{m v}$ than elements with smaller values of $k_{1}$ since constant $k_{1}$ curves with larger $k_{1}$ values exceed constant $k_{1}$ curves with smaller $k_{1}$ values. Third, five ranges are revealed where the curves exhibit very different characteristics. For small values of $\tau_{1}$, all of the curves 
have values of $\omega_{m v} \approx \omega_{v}$. In this range the Maxwell element behaves like a pure damper, therefore $\omega_{m \nu}$ is insensitive to $\tau_{1}$. Where the curves begin to deviate from $\omega_{\nu}$, the element behaves like a Type A element - some spring motion occurs and only slight changes in $\omega_{\mathrm{mv}}$ occur to changes in $\tau_{1}$. In the range where $\omega_{\mathrm{mv}}$ is very sensitive to $\tau_{1}$, the Maxwell element is Type $B$, neither damping nor stiffness dominant. In the range where $\omega_{\mathrm{mv}}$ decreases slightly and approaches a constant value as $\tau_{1}$ increases, the element is Type $C$ - stiffness dominant. Finally, for large values of $\tau_{1}$, the curves reach a constant value. In this range the Maxwell element behaves like a pure spring. Notice, unlike the range of small $\tau_{1}$ where $\omega_{m v} \approx \omega_{v}$ for all of the curves, in the range of large $\tau_{1}$, each curve approaches a unique value of $\omega_{m v}>\omega_{v}$.

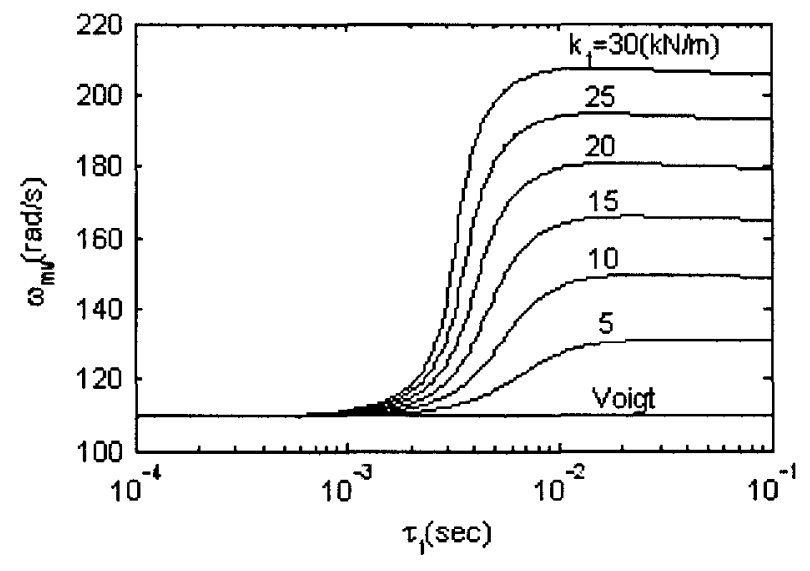

Fig. 3.2. Influence of $k_{1}$ and $\tau_{1}$ on $\omega_{m v}$.

It should be noted that the basic trends in the results presented in this article are similar for models with different values for $k$ and $c$. However, slight variations in the shapes of the curves exist. For example, Fig. 3.3 illustrates a curve for a M-V model 
where $k$ is $200 \mathrm{kN} / \mathrm{m}$ and $c$ is $300 \mathrm{~N}-\mathrm{s} / \mathrm{m}$. As can be seen, the characteristics of the curves are similar to the previous model although slight differences exist. For instance, the range where Type $A$ exists and the rate at which $\omega_{m v}$ reaches a constant value for increasing $\tau_{1}$ in the Type $C$ range differ.

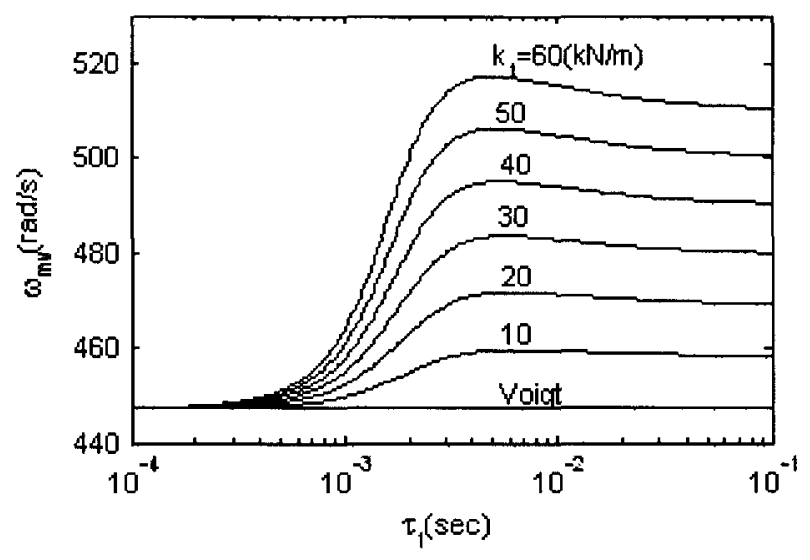

Fig. 3.3. Influence of $k_{1}$ and $\tau_{1}$ on $\omega_{m v}$ for a model with $k=200 \mathrm{kN} / \mathrm{m}$ and $c=300 \mathrm{~N}-\mathrm{s} / \mathrm{m}$.

Returning back to the original model, results will next be illustrated by varying $c_{1}$ in finite increments while $\tau_{1}$ varies continuously. Figure 3.4 illustrates constant $c_{1}$ curves where increasing $\tau_{1}$ is a result of decreasing $k_{1}$. The figure illustrates that for particular values of $\tau_{1}$, maximum values for $\omega_{m v}$ exist. These maximum values of $\omega_{m v}$ increase and the respective values of $\tau_{1}$ corresponding to maximum $\omega_{m v}$ decrease slightly as $c_{1}$ increases. For small values of $\tau_{1}$, all of the curves have values of $\omega_{m v} \approx \omega_{v}$. This is expected since the element behaves like a pure damper for small values of $\tau_{1}$ and hence $\omega_{m v}$ is insensitive to changes in $\tau_{1}$. Where the curves begin to deviate from $\omega_{v}$, the element behaves like a Type A element - some spring motion occurs and small changes in $\omega_{m v}$ occur to changes in $\tau_{1}$. In the range where $\omega_{\mathrm{mv}}$ is most sensitive to changes in $\tau_{1}$, the 
Maxwell element is Type B, neither damping nor stiffness dominates. The Maxwell element is Type $C$ - stiffness dominant - in the range of $\tau_{1}$ right of peak $\omega_{m v}$. Finally, for large values of $\tau_{1}$, the curves return to $\omega_{v}$ where the Maxwell element behaves like a pure spring whose stiffness tends to zero.

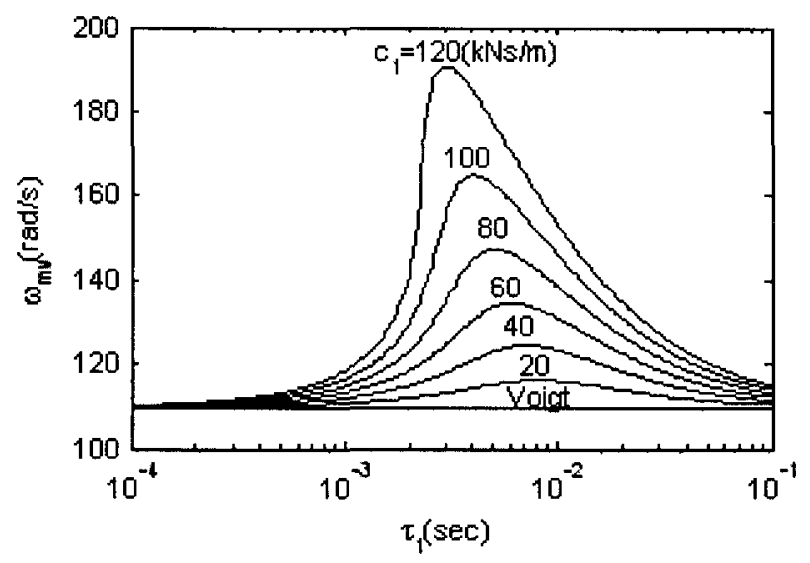

Fig. 3.4. Influence of $c_{1}$ and $\tau_{1}$ on $\omega_{m v}$.

A cluster of intersection points can be determined by drawing horizontal lines of constant $\omega_{m v}$ intersecting the curves in Fig. 3.2 or 3.4. Figure 3.5 plots the resulting cluster of points on the plane with coordinates of $k_{1}$ and $c_{1}$. These curves represent constant $\omega_{m v}$ values of 120 through $160 \mathrm{rad} / \mathrm{s}$ in steps of $10 \mathrm{rad} / \mathrm{s}$. In general, each curve has two branches. The Maxwell elements belonging to Type A exist on the lower branches, Type $C$ on the vertical branches and Type $B$ on the elbows of these curves. The graph illustrates that if the Maxwell element is Type A, the relationship between $k_{1}$ and $c_{1}$ is approximately linear for constant $\omega_{m v}$. If the Maxwell element is Type $C, \omega_{m v}$ depends solely on $k_{1}$ and increases as $k_{1}$ increases, while changes to $c_{1}$ have no influence $\omega_{m v}$. The 
constant $\tau_{1}$ lines (dotted) are provided to give some indication of the boundaries between element Types $A, B$ and $C$.

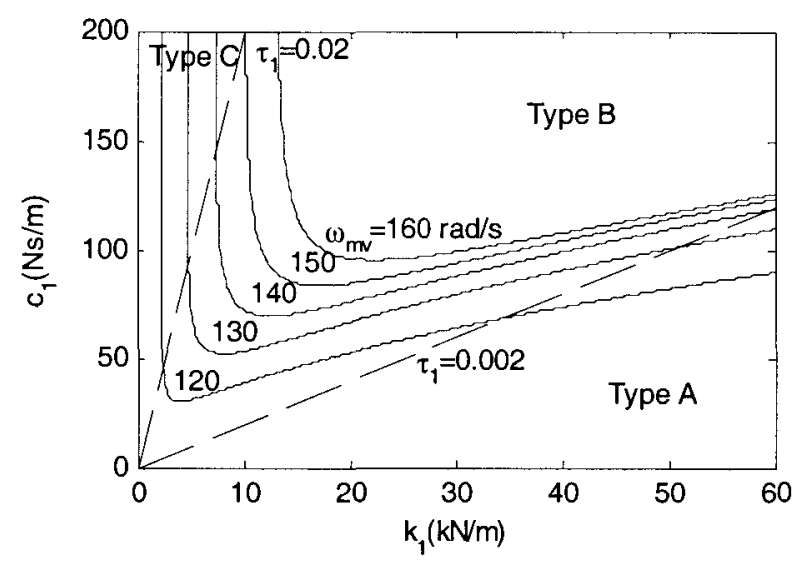

Fig. 3.5. Curves of constant $\omega_{m v}$.

\subsubsection{The Influences of $k_{1}$ and $c_{1}$ on damping ratio}

Figure 3.6 contains constant $k_{1}$ curves illustrating changes in the damping ratio $\zeta_{m v}$ of the M-V model as a function of $\tau_{1}$. Again, since $k_{1}$ is constant for each curve, changes in $\tau_{1}$ are proportional to changes in $c_{1}$. From the results, the following conclusions can be drawn. First, $\zeta_{m v}$ is not always greater than the damping ratio of the Voigt model $\zeta_{\nu}$ and does not always increase as $\tau_{1}$ increases. For a particular value of $\tau_{1}$, a maximum value of $\zeta_{m v}$ exists. This maximum value increases and the respective value of $\tau_{1}$ corresponding to maximum $\zeta_{m v}$ decreases slightly as $k_{1}$ increases. For small values of $\tau_{1}$, the Maxwell element behaves like a pure damper with $\zeta_{m v} \approx \zeta_{v}$. As $\tau_{1}$ increases, the element becomes Type $A$ and the curves begin to deviate from $\zeta_{v}$. In the range where $\zeta_{m v}$ is very sensitive to $\tau_{1}$, the Maxwell element is Type $B$. The damping ratio $\zeta_{m v}$ increases as 
$\tau_{1}$ increases when the Maxwell element is closest to being damping dominant (left of peak $\left.\zeta_{m v}\right)$, but decreases as $\tau_{1}$ increases when the Maxwell element is closest to being stiffness dominant (right of peak $\zeta_{m v}$ ). Of particular interest here is that a range of $\tau_{1}$ exists where $\zeta_{m v}<\zeta_{v}$, i.e., the damping ratio of the $\mathrm{M}-\mathrm{V}$ model is actually less than the damping ratio of the Voigt model. In this range the element is stiffness dominant (Type C). Finally, for large values of $\tau_{1}$, the curves reach a constant value. In this range the Maxwell element behaves like a pure spring. Note, unlike the range of small $\tau_{1}$ where $\zeta_{m v}$ $\approx \zeta_{v}$ for all the curves, in the range of large $\tau_{1}$, each curve approaches a unique value $\zeta_{m v}$ $<\zeta_{v}$ which depends on $k_{1}$.

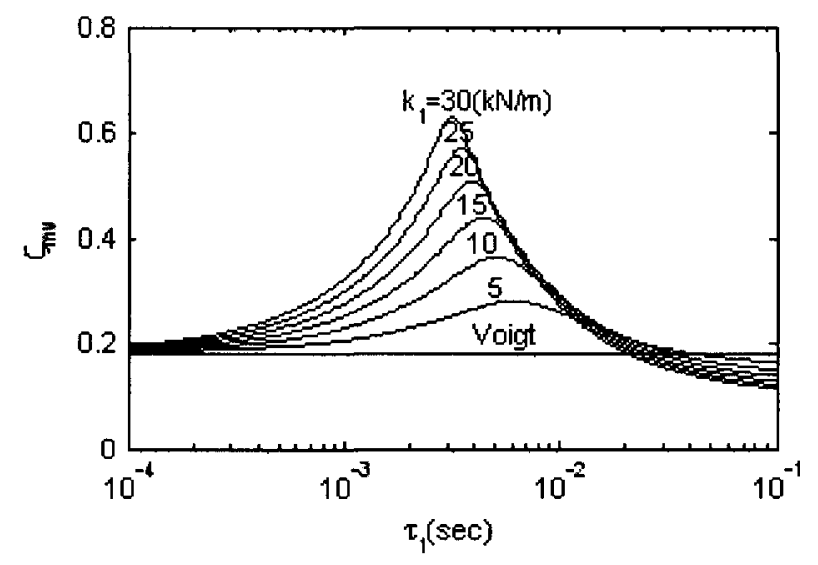

Fig. 3.6. Influence of $k_{1}$ and $\tau_{1}$ on $\zeta_{m v}$.

Next, observe what happens if $c_{1}$ varies in increments and $\tau_{1}$ varies continuously. Figure 3.7(a) illustrates constant $c_{1}$ curves where increasing $\tau_{1}$ is a result of decreasing $k_{1}$ and Fig. 3.7(b) is a magnification of the range $0.03 \mathrm{sec}<\tau_{1}<30 \mathrm{sec}$. As with the other results, different ranges can be observed from Fig. 3.7 where characteristics of the curves 
are unique for each range. For small values of $\tau_{1}$, the Maxwell element behaves like a pure damper; therefore, $\zeta_{m v} \neq \zeta_{\nu}$ and $\zeta_{m v}$ depends only on $c_{1}$. Where the curves begin to deviate from their original values, the element behaves like a Type A element - some spring motion occurs and only slight changes in $\zeta_{m v}$ result from changes in $\tau_{1}$. In the range where $\zeta_{m v}$ is very sensitive to $\tau_{1}$, the Maxwell element is Type $B$. Where $\zeta_{m v}$ drops below $\zeta_{\nu}$ and then increases approaching $\zeta_{\nu}$ the element is Type $C$-stiffness dominant. Above this range the Maxwell element behaves like a pure spring whose stiffness tends to zero and eventually for large $\tau_{1}$ the Maxwell element has no influence on the system, therefore $\zeta_{m v}=\zeta_{v}$. 

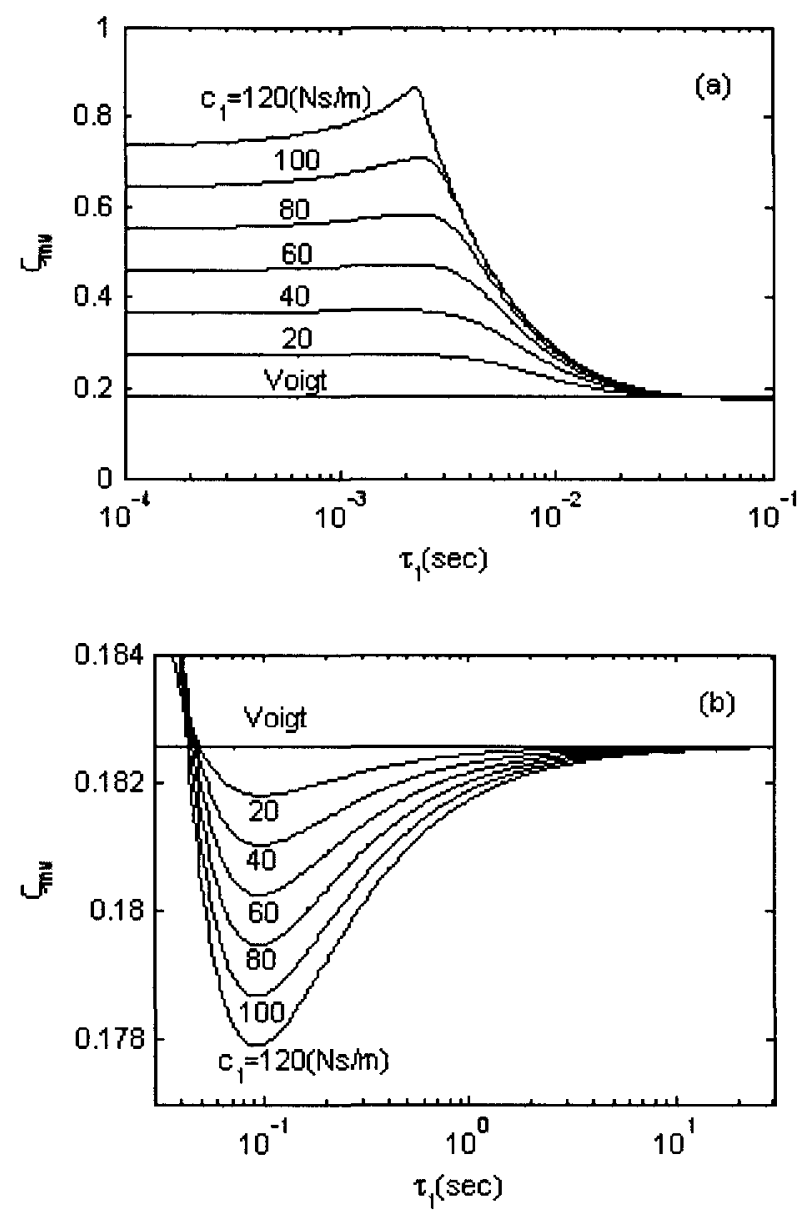

Fig. 3.7. Influence of $c_{1}$ and $\tau_{1}$ on $\zeta_{m v}$.

A cluster of intersection points can be determined by drawing horizontal lines of constant $\zeta_{m v}$ curves from Fig. 3.6 or 3.7. Figure 3.8 plots the resulting cluster of points on the plane with coordinates of $k_{1}$ and $c_{1}$. In general, there are two groups of curves. The first group exits in the lower-right section of the plane where $\zeta_{m v}>\zeta_{\nu}$. The second group exists in the upper-left section where $\zeta_{m v}<\zeta_{v}$. Also, each curve consists of two branches. Maxwell elements belonging to Type $A$ are found on the horizontal branches of the first group where changes to $k_{1}$ do not influence $\zeta_{m v}$. Therefore $\zeta_{m v}$ depends solely on $c_{1}$ (the 
Maxwell element is damping dominant). Maxwell elements belonging to Type $B$ exist on the upper branches and elbows of the curves of the first group. Finally, Maxwell elements belonging to Type $C$ are represented by the second group of curves.

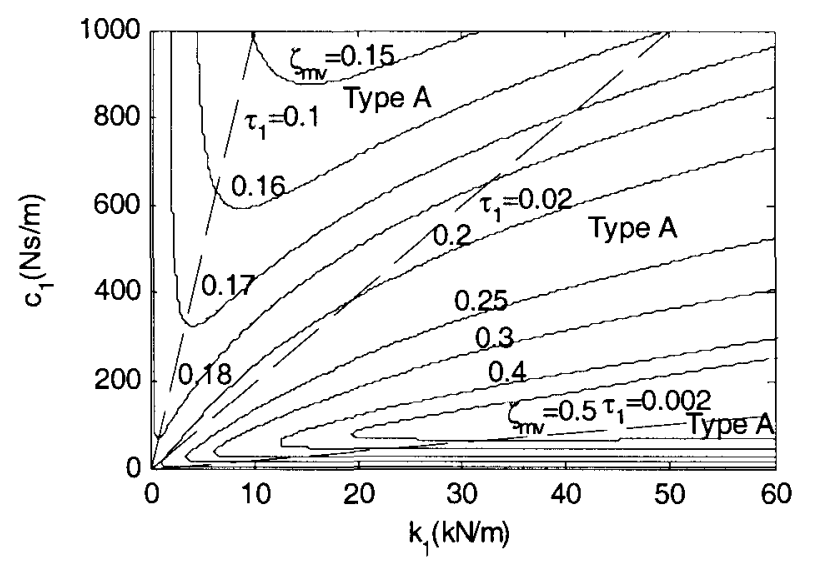

Fig. 3.8. Curves of constant $\zeta_{m v}$.

\subsection{Harmonic Analysis}

In this section the influences of $k_{1}$ and $c_{1}$ on the frequency response of the single mass system are investigated. Figure 3.9 illustrates frequency response spectra of three Voigt models whose parameters are listed in Table 3.2, referred to hereafter as Voigt Model I, II, and III. These sample models and their corresponding frequency response functions will be useful in the subsequent analysis. 


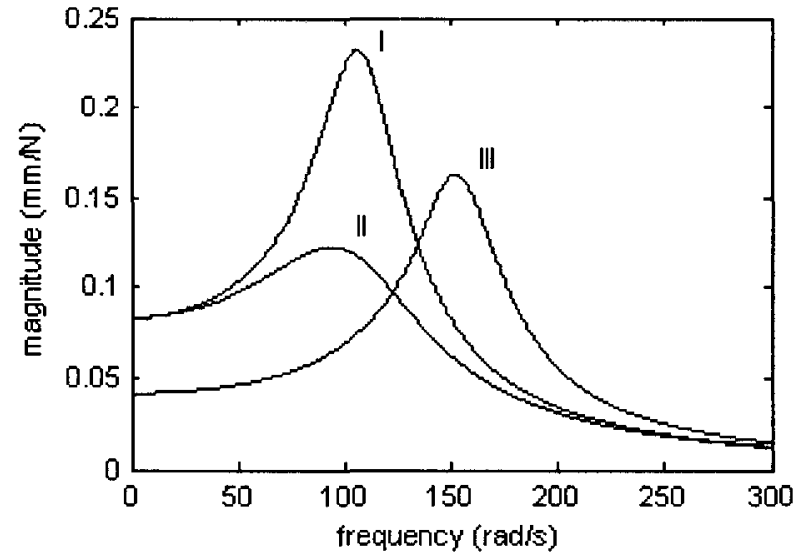

Fig. 3.9. Frequency response spectra of three Voigt models.

Table 3.2. Parameters of three Voigt models.

\begin{tabular}{|c|c|c|c|}
\hline Voigt Model & $I$ & $I I$ & $I I I$ \\
\hline$m(\mathrm{~kg})$ & 1 & 1 & 1 \\
\hline$k_{v}(\mathrm{kN} / \mathrm{m})$ & 12 & 12 & 24 \\
\hline$c_{v}(\mathrm{~N}-\mathrm{s} / \mathrm{m})$ & 40 & 80 & 40 \\
\hline
\end{tabular}

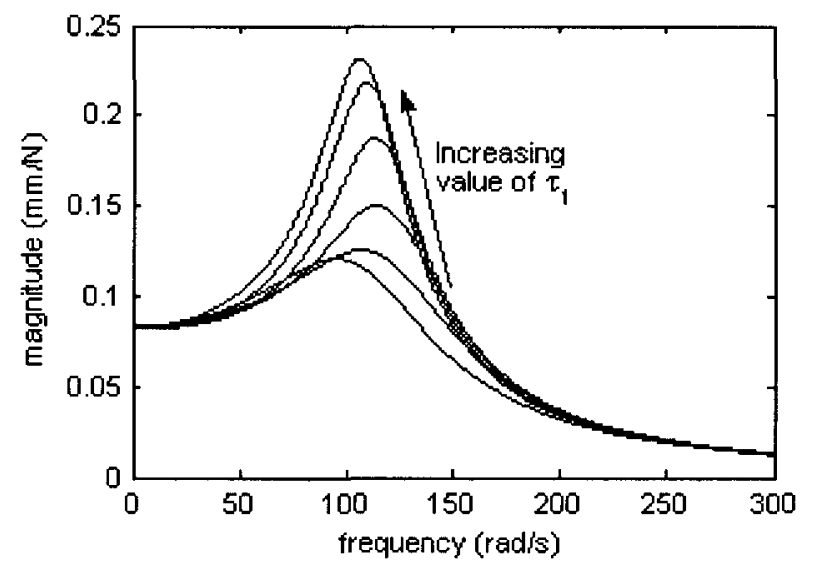

Fig. 3.10. The influence of $\tau_{1}$ ( $c_{1}$ is constant) on $M-V$ frequency response model. 
Consider M-V models where $m, k$ and $c$ are the same as Voigt Model I in Table $3.2, c_{1}$ is $40 \mathrm{~N}-\mathrm{s} / \mathrm{m}$ and $\tau_{1}$ increases from 0.001 to $0.5 \mathrm{sec}$. The resulting frequency response specta are shown in Fig. 3.10. The Figure illustrates that $\omega_{m v}$ changes only slightly compared with the peak amplitude. The amplitude of the peak increases and shifts slightly up in frequency as $\tau_{1}$ increases when $\tau_{1}$ is small. Here, the Maxwell element changes from Type A to Type B. For larger values of $\tau_{1}$, the peak continues to increase in amplitude and shifts down in frequency where the Maxwell element changes from Type B to Type $C$. These results are consistent with the findings of section 3.2. As $\tau_{1}$ tends to infinity, $k_{1}$ tends to zero and the influence from the Maxwell element diminishes. Therefore, the frequency response matches the frequency response of Voigt Model I illustrated in Fig. 3.10. As $\tau_{1}$ tends to zero, $k_{1}$ tends to infinity and the Maxwell element behaves like a pure damper in parallel with the primary damper. Therefore, the total damping approaches $c+c_{1}=80 \mathrm{~N}$-s/m, which is the damping value for Voigt Model $I I$, and the frequency response of the $\mathrm{M}-\mathrm{V}$ model matches the frequency response of Voigt Model II.

Next, let $k_{1}$ equal $12 \mathrm{kN} / \mathrm{m}$ while $\tau_{1}$ increases from 0.0005 to $5 \mathrm{sec}$. Sample frequency response spectra are illustrated in Fig. 3.11, where it is shown that as $\tau_{1}$ increases $\omega_{m \nu}$ changes only slightly at first while the peak magnitude decreases. In this case the Maxwell element is Type A. When the peak amplitude is close to the static response amplitude, the peak begins to move right with increasing $\tau_{1}$. For this range of $\tau_{1}$ the Maxwell element is Type B. Notice that the portion of the frequency response left of the peak drops below the static response amplitude. Consequently, isolation can be achieved below $\omega_{m v}$, which is a phenomenon that a Voigt model is incapable of capturing. 
As $\tau_{1}$ continues to increase, the peak increases. In this range the Maxwell element is Type $C$. These results are all consistent with the findings of section 3.2. As $\tau_{1}$ tends to infinity, $c_{1}$ also approaches infinity and the Maxwell element behaves like a pure spring in parallel with the primary spring. Therefore, the total stiffness approaches $k+k 1=24 \mathrm{kN} / \mathrm{m}$, which is stiffness of Voigt Model III. Consequently, the frequency response approaches the frequency response of Voigt Model III in Fig.3.9. As $\tau_{1}$ tends to zero, $c_{1}$ tends to zero and the frequency response approaches the frequency response of Voigt Model I in Fig. 3.9.

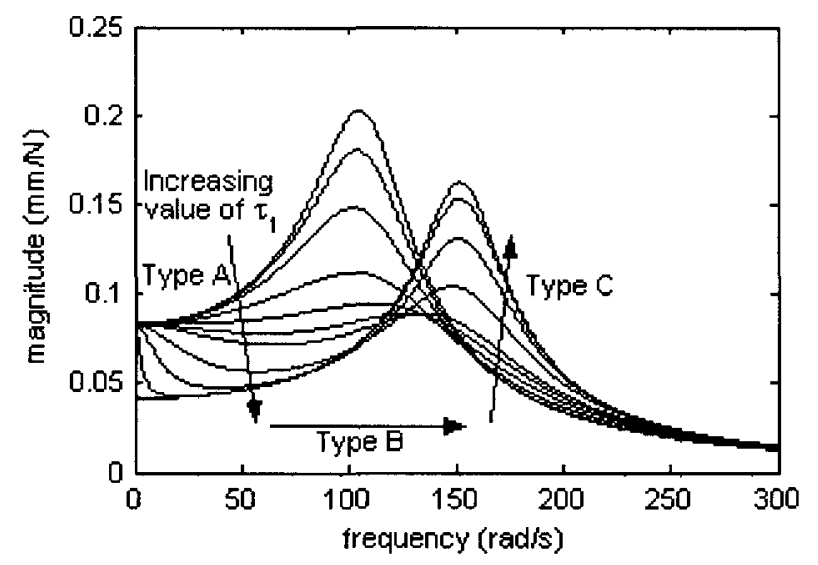

Fig. 3.11. The influence of $\tau_{1}\left(k_{1}\right.$ is constant) on $\mathrm{M}-\mathrm{V}$ frequency response model.

\subsection{Parameter Identification}

For the parameter identification, assume that the three $M-V$ systems listed in Table 3.3 are systems who's frequency response spectra are available from vibration experiment. The "experimental" systems are denoted as Maxwell System $A, B$ and $C$ corresponding to the type of Maxwell element possessed by each system. Also listed are the natural frequencies and damping ratios of these systems. In practice, if the frequency 
response spectra of these systems were experimentally measured, then the parameters $m$, $c, k, c_{1}, k_{1}$ of $\mathrm{M}-\mathrm{V}$ models for representing these systems are unknown model parameters to be determined. Before discussing the procedure for determining these parameters, first consider identifying Voigt models of these systems. From modal parameter estimation, the natural frequencies $\omega_{\nu}$ and damping ratios $\zeta_{\nu}$ of the Voigt models are identified from the measured frequency response spectra. Therefore, $\omega_{v}=\omega_{m v}$ and $\zeta_{v}=\zeta_{m v}$. Then, two approaches exist to identify $m_{v}, c_{v}$ and $k_{v}$ of the Voigt models. Either the mass could be measured on a weight scale, then $m_{v}=m, k_{v}=m_{v} \omega_{n}{ }^{2}$ and $c_{v}=2 m_{v} \zeta \omega_{n}$. Or, the stiffness could be determined from the DC component of the frequency response or from a static stiffness experiment. Then, $k_{v}=k, m_{v}=k_{v} / \omega_{n}{ }^{2}$ and $c_{v}=2 m_{v} \zeta \omega_{n}$. The Voigt model identified by the first approach will be referred to as Voigt Model $M$ - the mass consistent Voigt model, and the Voigt model identified by the second approach will be referred to as Voigt Model $K$ - the stiffness consistent Voigt model. The corresponding parameters of the Voigt models identified from the frequency response spectra of the three different “experimental" M-V systems are listed in Table 3.4. 
Table 3.3. Parameters of "Experimental" M-V systems.

\begin{tabular}{|c|c|c|c|}
\hline Parameter & M-V System A & M-V System B & M-V System C \\
\hline$m(\mathrm{~kg})$ & 1 & 1 & 1 \\
\hline$k(\mathrm{kN} / \mathrm{m})$ & 12 & 12 & 12 \\
\hline$c(\mathrm{~N}-\mathrm{s} / \mathrm{m})$ & 40 & 40 & 40 \\
\hline$k_{1}(\mathrm{kN} / \mathrm{m})$ & 12 & 12 & 12 \\
\hline$c_{1}(\mathrm{~N}-\mathrm{s} / \mathrm{m})$ & 20 & 80 & 400 \\
\hline$\omega_{m v}(\mathrm{rad} / \mathrm{s})$ & 111.56 & 145.14 & 156.16 \\
\hline$\zeta_{m v}$ & 0.28 & 0.36 & 0.18 \\
\hline
\end{tabular}

Table 3.4. Parameters of identified Voigt models.

\begin{tabular}{|c|c|c|c|c|}
\hline \multicolumn{2}{|c|}{ Parameter } & M-V System A & M-V System B & M-V System C \\
\hline \multirow{3}{*}{ Voigt } & $m_{v}(\mathrm{~kg})$ & 1 & 1 & 1 \\
\cline { 2 - 5 } Model $M$ & $k_{v}(\mathrm{kN} / \mathrm{m})$ & 12.45 & 21.07 & 24.38 \\
\cline { 2 - 5 } & $c_{v}(\mathrm{~N}-\mathrm{s} / \mathrm{m})$ & 61.45 & 104.56 & 55.25 \\
\hline \multirow{3}{*}{$\begin{array}{c}\text { Voigt } \\
\text { Model } K\end{array}$} & $m_{v}(\mathrm{~kg})$ & 0.96 & 0.57 & 0.49 \\
\cline { 2 - 5 } & $k_{v}(\mathrm{kN} / \mathrm{m})$ & 12 & 12 & 12 \\
\cline { 2 - 5 } & $c_{v}(\mathrm{~N}-\mathrm{s} / \mathrm{m})$ & 59.25 & 59.56 & 27.19 \\
\hline
\end{tabular}

Figure 3.12(a-c) shows the frequency response functions of the "experimental" $\mathrm{M}-\mathrm{V}$ systems as well as the corresponding identified Voigt Models $M$ and $K$ of these systems. For all three graphs, the solid curve with the smaller amplitude is the frequency 
response of the identified Voigt Model $M$, the solid curve with the larger amplitude is the frequency response of the identified Voigt Model $K$, and the dashed curve is the frequency response of the "experimental" M-V system. Note that for M-V System A (Fig. 3.12(a)), the frequency response spectra of the two identified Voigt models are not very different and that the frequency response of the identified Voigt Model $K$ is closer to that of the actual M-V system (this is not visible due to the closeness of the two curves). For the M-V System B (Fig. 3.12(b)), the frequency response spectra of two identified Voigt models are very different. The frequency response of the identified Voigt Model $K$ is closer to that of the M-V system in the low frequency range, while the frequency response of the identified Voigt Model $M$ is closer to that of the M-V system in the high frequency range. For the M-V System $C$ (Fig. 3.12(c)), the frequency response spectra of the two identified Voigt models are also very different. Here, the frequency response of the identified Voigt Model $M$ is closer to that of the M-V system except in the low frequency range. 

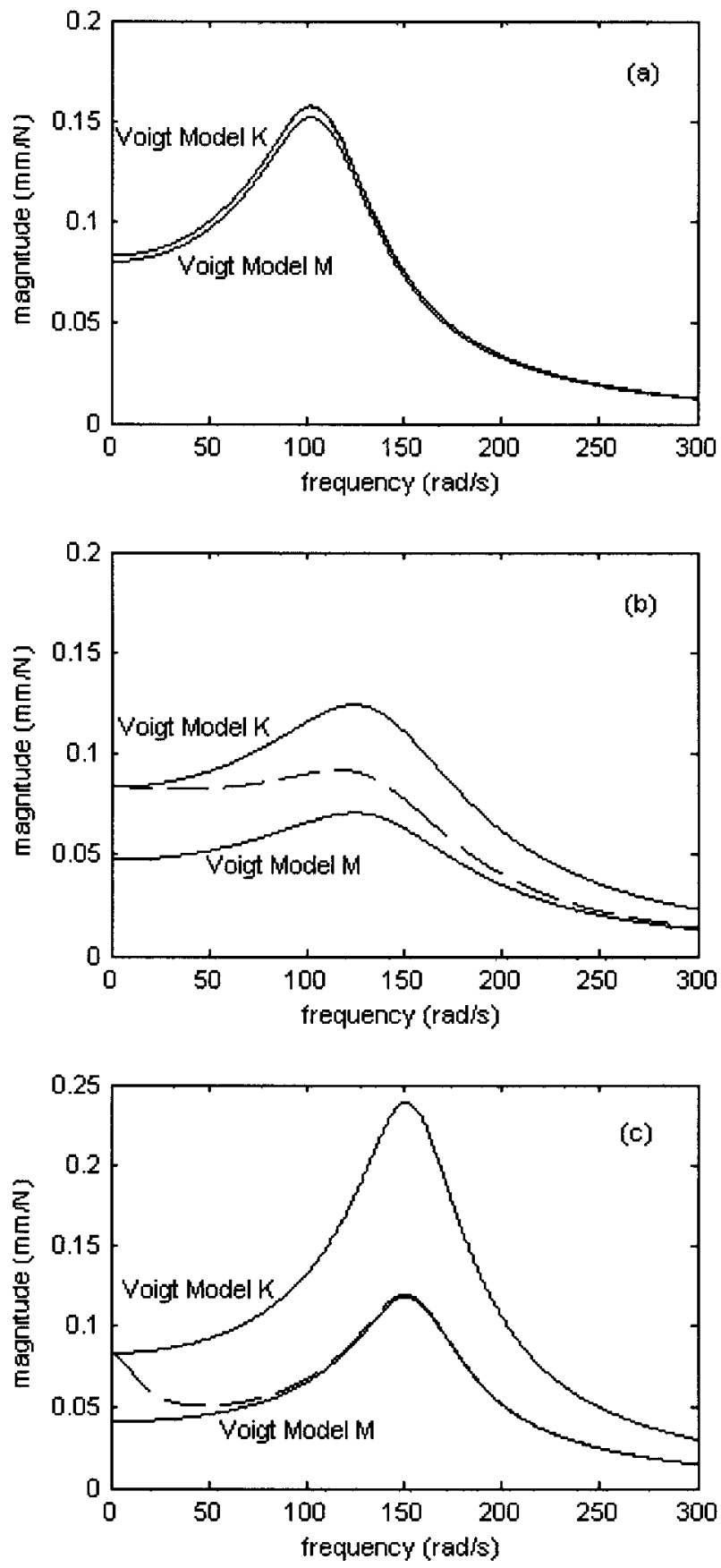

Fig.3.12. Frequency response spectra of Voigt Models $M$ and $K$ and "experimental" M-V systems (solid line) with equivalent natural frequencies and damping ratios. 
Figure 3.12 illustrates that Voigt models having equivalent natural frequencies and damping ratios as $\mathrm{M}-\mathrm{V}$ systems may not match the frequency response functions of the M-V systems over the entire frequency range. This is especially true when identifying a Voigt model to represent an $\mathrm{M}-\mathrm{V}$ system possessing characteristics of a Type $B$ Maxwell element. Therefore, returning to the identification of M-V models of these systems, the goal is to identify the parameters $m, c, k, c_{1}$ and $k_{1}$. As with the identification of the Voigt models, $m$ can be determined on a weight scale and $k$ can either be estimated from the DC component of the frequency response function or from static stiffness experiment. Therefore, only $c, c_{1}$ and $k_{1}$ are left to be determined. By estimating $\omega_{m v}$ and $\zeta_{m v}$ from the measured frequency response spectra by experimental modal analysis, $c_{1}$ and $k_{1}$ can be identified by generating constant $\omega_{m v}$ and $\zeta_{m v}$ curves of the model to be identified as shown in Fig. 3.5 and 3.8, respectively. By graphing these curves on same figure, the intersection of the two curves yields identified values for $k_{1}$ and $c_{1}$. For example, for the M-V System $A$ in Table 3.3, Fig. 3.13 illustrates two sets of constant $\omega_{m v}$ and $\zeta_{m v}$ curves of a M-V Model $A$ with $c$ equal to 20 and $40 \mathrm{~N}-\mathrm{s} / \mathrm{m}$. Note that the constant natural frequency curves are roughly the same for the two values chosen for $c$. Where the two sets of curves intersect, two potential sets of values for $c_{1}$ and $k_{1}$ exist for the M-V Model A, both of which give the same natural frequency and damping ratio. So the question is, which resulting model is more accurate? This question leads to the final step in the identification process, completed by generating a family of frequency response spectra for different values of $c$ and corresponding $c_{1}$ and $k_{1}$. 


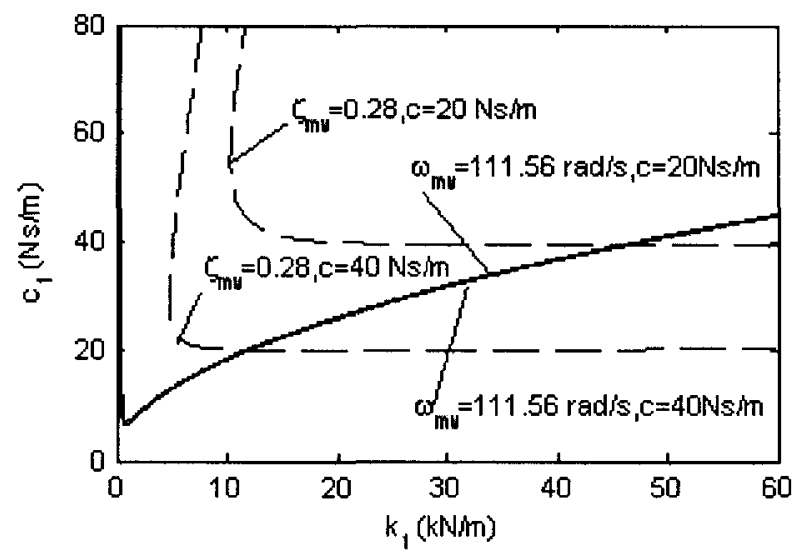

Fig. 3.13. Identifying $k_{1}$ and $c_{1}$ from constant $\omega_{m v}$ and $\zeta_{m v}$ curves.

For the identification of the M-V System A, Table 3.5 lists sample values of $c_{1}$ and $k_{1}$ for the M-V Model $A$ to be identified for this system by varying $c$ from -80 to $60 \mathrm{~N}$ $\mathrm{s} / \mathrm{m}$. The resulting frequency response spectra of these groups of parameters are shown in Fig. 3.14. All the frequency response functions have equivalent $\omega_{m v}$ and $\zeta_{m v}$; however, as $c$ increases, the peak decreases as the Maxwell element of M-V Model A changes from Type $A$ to Type $B$ and then to Type $C$. The frequency response spectra with $c$ equal to 40 $\mathrm{N}-\mathrm{s} / \mathrm{m}$ is closest to the actual frequency response spectra of the M-V System $A$. Therefore, from this identification process, the results from the group of parameters where $c$ is $40 \mathrm{~N}$ s/m would be chosen for the M-V Model A to represent the M-V System A. 
Table 3.5. Parameters of M-V Model A for M-V System A.

\begin{tabular}{|c|c|c|}
\hline$c(\mathrm{~N}-\mathrm{s} / \mathrm{m})$ & $c_{1}(\mathrm{~N}-\mathrm{s} / \mathrm{m})$ & $k_{1}(\mathrm{kN} / \mathrm{m})$ \\
\hline-80 & 139.36 & 551.23 \\
\hline 0 & 59.51 & 102.25 \\
\hline 20 & 39.63 & 45.93 \\
\hline 40 & 19.99 & 11.99 \\
\hline 60 & 10.26 & 0.41 \\
\hline
\end{tabular}

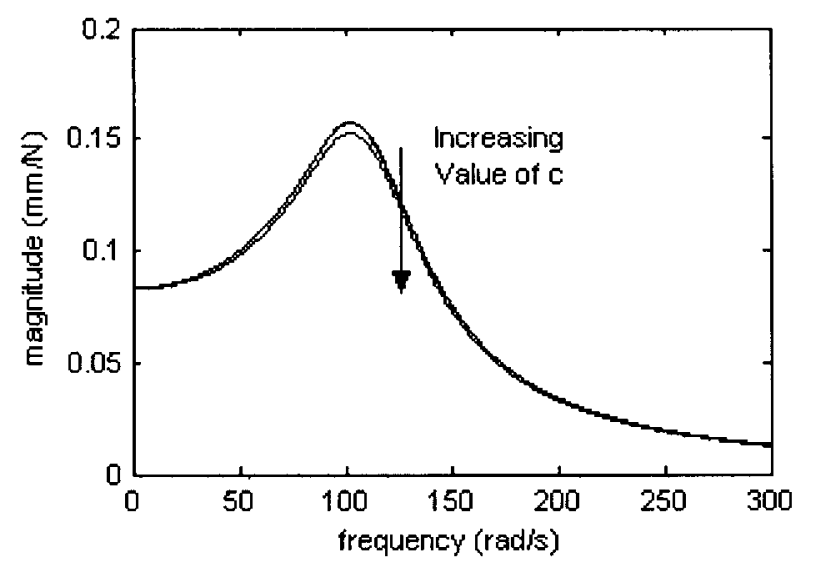

Fig. 3.14. Frequency response spectra of M-V Model A.

Note that when $c$ is equal to $60 \mathrm{~N}$-s/m, the Maxwell element is Type $C$-stiffness dominant. Therefore, M-V Model $A$ model is approximately a Voigt model with $c_{v}=c=$ $60 \mathrm{~N}-\mathrm{s} / \mathrm{m}$ and $k_{v}=k+k_{1}=12.41 \mathrm{kN} / \mathrm{m}$. These values are similar to the values of the identified Voigt Model $M$ in Table 3.4, where $c_{v}=61.45 \mathrm{~N}-\mathrm{s} / \mathrm{m}$ and $k_{v}=12.45 \mathrm{kN} / \mathrm{m}$ whose frequency response is the lower curve in Fig. 3.12(a). For values of $c$ greater than 61.45 N-s/m for M-V Model A, the constant $\omega_{m v}$ curve and constant $\zeta_{m v}$ curve do not intersect. This implies that the frequency response spectrum of Voigt Model $M$ is the 
lower limit for the frequency response of this $\mathrm{M}-\mathrm{V}$ model. No parameters exist for $\mathrm{M}-\mathrm{V}$ Model $A$ that yield the same natural frequency and damping ratio under this limit.

When $c$ is zero, even negative, intersection points of the constant $\omega_{m v}$ and constant $\zeta_{m v}$ curves for M-V Model $A$ exist, and the frequency response amplitude increases as $c$ decreases. However, the differences in amplitude diminish as $c$ continues to decrease and another limit is found. This limit is the frequency response of Voigt Model K in Fig. 3.12(a). To illustrate this, note that for $c$ equal to $-80 \mathrm{~N}-\mathrm{s} / \mathrm{m}$, the Maxwell element of M-V Model A is Type A - damping dominant - and the model is similar to a Voigt model with $c_{v}=c+c_{1}=-80+139.36=59.36 \mathrm{~N}-\mathrm{s} / \mathrm{m}$ and $k_{v}=k=12 \mathrm{kN} / \mathrm{m}$. These values are approximately the values for Voigt Model $K$ in Table 3.4. As $c$ of M-V Model A continues to decrease, its frequency response continues to approach that of Voigt Model $K$ in Fig. 3.12(a), although it is never exceeded.

Next, consider identifying the M-V System B in Table 3.3. Table 3.6 lists sample values of $c_{1}$ and $k_{1}$ for the M-V Model $B$ to be identified for this system by changing $c$ from -240 to $100 \mathrm{~N}-\mathrm{s} / \mathrm{m}$. The resulting frequency response spectra of these groups of parameters are shown in Fig. 3.15. Similar to what was observed in identifying $M-V$ Model $A$, all the frequency response spectra have the same $\omega_{m v}$ and $\zeta_{m v}$. However, as $c$ increases, the amplitude of frequency response decreases as the Maxwell element of M-V Model $B$ changes from Type $A$ to Type $B$ and then to Type $C$. The frequency response spectrum with $c$ equal to $40 \mathrm{~N}-\mathrm{s} / \mathrm{m}$ is closest to the actual frequency response of the $\mathrm{M}-\mathrm{V}$ System $B$. Therefore, the identified results from the group of parameters where $c$ is $40 \mathrm{~N}$ $\mathrm{s} / \mathrm{m}$ would be chosen for the M-V Model B to represent the M-V System B. 
Table 3.6. Parameters of M-V Model B for M-V System B.

\begin{tabular}{|c|c|c|}
\hline$c(\mathrm{~N}-\mathrm{s} / \mathrm{m})$ & $c_{1}(\mathrm{~N}-\mathrm{s} / \mathrm{m})$ & $k_{1}(\mathrm{kN} / \mathrm{m})$ \\
\hline-240 & 310.89 & 248.90 \\
\hline-80 & 160.7 & 68.92 \\
\hline 0 & 96.88 & 23.54 \\
\hline 40 & 80.00 & 12.00 \\
\hline 80 & 138.43 & 7.90 \\
\hline 100 & 815.13 & 8.64 \\
\hline
\end{tabular}

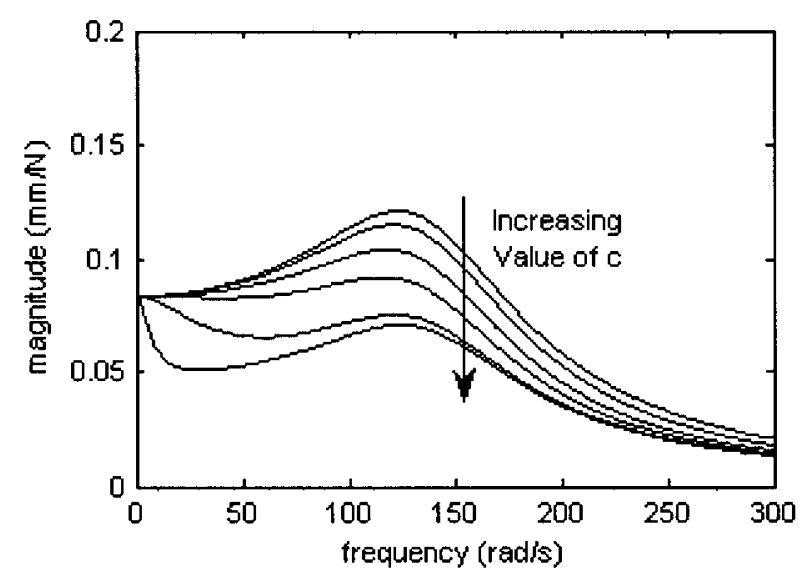

Fig. 3.15. Frequency response functions of M-V Model B.

When $c$ is $100 \mathrm{~N}-\mathrm{s} / \mathrm{m}$, the Maxwell element is Type $C$ - stiffness dominant - and M-V Model $B$ is approximately a Voigt model with $c_{v}=c=100 \mathrm{~N}-\mathrm{s} / \mathrm{m}$ and $k_{v}=k+k_{1}=$ $20.64 \mathrm{kN} / \mathrm{m}$. These values are similar to the values of the identified Voigt Model $M$ in Table 3.4, where $c_{v}=104.56 \mathrm{~N}-\mathrm{s} / \mathrm{m}$ and $k_{v}=21.07 \mathrm{kN} / \mathrm{m}$ whose frequency response is the lower curve in Fig. 3.12(b). For values of $c$ greater than $104.56 \mathrm{~N}-\mathrm{s} / \mathrm{m}$ of the M-V Model $B$, the constant $\omega_{m v}$ curve and constant $\zeta_{m v}$ curve do not intersect. This implies that the 
frequency response spectrum of the Voigt Model $M$ is the lower limit of frequency response of this M-V model. No parameters exit for M-V Model B that yield the same natural frequency and damping ratio under this limit.

When $c$ is zero, even negative, intersection points of the constant $\omega_{m v}$ and constant $\zeta_{m v}$ curves for M-V Model $B$ exist, and the frequency response amplitude increases as $c$ decreases. However, the differences diminish as $c$ decreases and another limit is again found. This limit is the frequency response of Voigt Model $K$ in Fig. 3.12(b). The frequency response of M-V Model B never exceeds this limit.

Lastly, consider the identification of the M-V Systems $C$ in Table 3.3. Table 3.7 lists sample values of $c_{1}$ and $k_{1}$ for the M-V Model $C$ to be identified for this system by changing $c$ from -320 to $55 \mathrm{~N}-\mathrm{s} / \mathrm{m}$. The resulting frequency response spectra of these groups of parameters are shown in Fig. 3.16. Similar to what was observed in identifying M-V Model $A$ and $B$, all the frequency response spectra have equivalent $\omega_{\mathrm{mv}}$ and $\zeta_{\mathrm{mv}}$. However, as $c$ increases, the frequency response amplitude decreases as the Maxwell element of M-V Model $C$ changes from Type A to Type B and then to Type $C$. The frequency response with $c$ equal to $40 \mathrm{~N}-\mathrm{s} / \mathrm{m}$ is closest to the actual frequency response of the M-V System C. Therefore, the identified results from the group of parameters where $c$ is $40 \mathrm{~N}-\mathrm{s} / \mathrm{m}$ would be chosen for the M-V Model C to represent the M-V System C. 
Table 3.7. Parameters of M-V Model C for M-V System C.

\begin{tabular}{|c|c|c|}
\hline$c(\mathrm{~N}-\mathrm{s} / \mathrm{m})$ & $c_{1}(\mathrm{~N}-\mathrm{s} / \mathrm{m})$ & $k_{1}(\mathrm{kN} / \mathrm{m})$ \\
\hline-320 & 363.95 & 268.90 \\
\hline-160 & 216.41 & 91.727 \\
\hline-80 & 153.69 & 40.93 \\
\hline-40 & 133.23 & 24.99 \\
\hline 0 & 141.04 & 15.34 \\
\hline 20 & 185.64 & 12.88 \\
\hline 40 & 399.70 & 12.00 \\
\hline 55 & 25357 & 12.37 \\
\hline
\end{tabular}

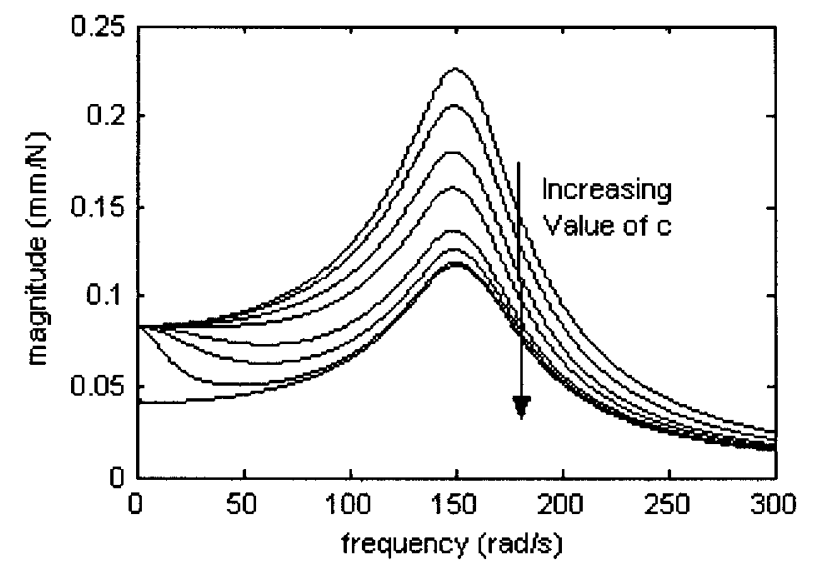

Fig. 3.16. Frequency response functions of M-V Model C.

When $c$ is $55 \mathrm{~N}$-s/m, the Maxwell element is Type $C$ - stiffness dominant - and the M-V model is approximately a Voigt model with $c_{\nu}=c=55 \mathrm{~N}-\mathrm{s} / \mathrm{m}$ and $k_{v}=k+k_{1}=$ $24.37 \mathrm{kN} / \mathrm{m}$. These values similar to the values of the identified Voigt Model $M$ in Table 
3.4 , where $c_{\nu}=55.25 \mathrm{~N}-\mathrm{s} / \mathrm{m}$ and $k_{\nu}=24.38 \mathrm{kN} / \mathrm{m}$ whose frequency response function is the lower curve in Fig. 3.13(c). For values of $c$ greater than $55.25 \mathrm{~N}-\mathrm{s} / \mathrm{m}$ of the M-V Model $C$, the constant $\omega_{m \nu}$ curve and constant $\zeta_{m \nu}$ curve do not intersect. This implies that the frequency response spectrum of the Voigt Model M is the lower limit of frequency response of this $\mathrm{M}-\mathrm{V}$ model. No parameter exists for $\mathrm{M}-\mathrm{V}$ Model $C$ that yield the same natural frequency and damping ratio under this limit.

When $c$ is zero, even negative, intersection points of the constant $\omega_{m v}$ and constant $\zeta_{m v}$ curves for $\mathrm{M}-\mathrm{V}$ Model $C$ exist, and the frequency response amplitude increases as $c$ decreases. However, the differences diminish as $c$ decreases and another limit is found. This limit is the frequency response spectrum of Voigt Model $K$ in Fig. 3.12(c). The frequency response of M-V Model C never exceeds this limit.

\subsection{Summary}

In this Chapter, modal analysis of a single mass elastomeric isolation system represented by a Maxwell-Voigt (M-V) model is conducted and the influences that the stiffness $k_{1}$ and damping $c_{1}$ of the Maxwell element have on the natural frequency and damping ratio of the $\mathrm{M}-\mathrm{V}$ model is revealed. Three types of Maxwell elements are defined and are distinct by their time constant, $\tau_{1}=c_{1} / k_{1}$. If $\tau_{1}$ is small, the Maxwell element is Type $A$-damping dominant. If $\tau_{1}$ is large, the Maxwell element is Type $C$ stiffness dominant. Falling between these two element types is the Type B Maxwell element - neither damping nor stiffness dominant. The different influences that each of these types have on the system natural frequencies and damping ratio is discussed. It is also shown that the existence of the Maxwell element in the M-V model always results in 
a natural frequency greater than a Voigt model with equivalent Voigt elements as the M$\mathrm{V}$ model. However, this is not necessarily true for the damping ratio. For certain Maxwell element types, the damping ratio can actually be less than the Voigt model.

Harmonic analysis reveals that $\mathrm{M}-\mathrm{V}$ models with unlike parameters can have the same natural frequency and damping ratio and different frequency response functions. Consequently, $\mathrm{M}-\mathrm{V}$ models are more capable of representing elastomeric isolation systems over a broad frequency range. This is unlike Voigt models whose frequency response functions are fixed once their natural frequency, damping ratio and either mass or stiffness are determined. Consequently, a parameter identification method is developed. The method is based upon constant natural frequency and damping ratio curves of a $\mathrm{M}-\mathrm{V}$ frequency response model. These curves are determined the parametric data yielded from the modal analysis. In addition, it is shown that the frequency response function of a M-V model exists between two limits. These limits are frequency response functions of two Voigt models, a mass consistent Voigt model (Voigt Model $M$ whose mass is equivalent to the mass of the M-V model) and a stiffness consistent Voigt model (Voigt Model $K$ whose stiffness is equivalent to the static stiffness of the M-V model). The effectiveness of the parameter identification method is verified by several analytical examples. 


\section{CHAPTER 4}

DYNAMIC ANALYSIS AND PARAMETER IDENTIFICATION OF GENERAL

MAXWELL MODEL

\subsection{Problem Formulation}

In this chapter the dynamic analysis and parameter identification method is investigated for Maxwell models having two or more Maxwell elements (Fig. 4.1).

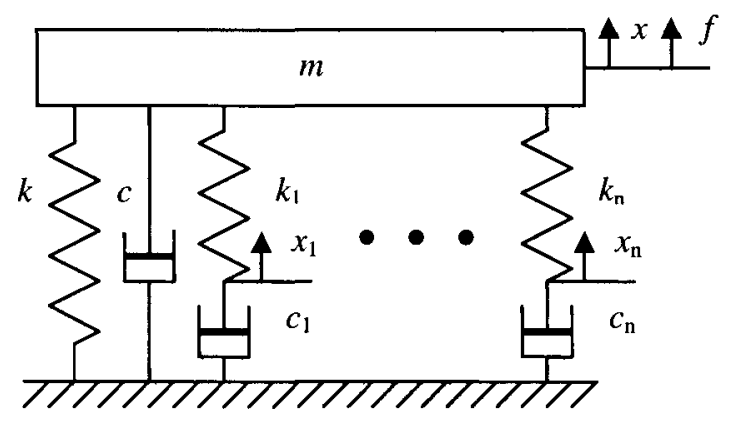

Fig. 4.1. Mathematic model of isolator represented by general Maxwell model.

The governing equations of motion for the general Maxwell system represented in Fig. 4.1 are, $\left[\begin{array}{ccccc}m & 0 & 0 & \cdots & 0 \\ 0 & 0 & 0 & \cdots & 0 \\ 0 & 0 & 0 & \cdots & 0 \\ \vdots & \vdots & \vdots & \ddots & \vdots \\ 0 & 0 & 0 & \cdots & 0\end{array}\right]\left\{\begin{array}{c}\ddot{x} \\ \ddot{x}_{1} \\ \ddot{x}_{2} \\ \vdots \\ \ddot{x}_{n}\end{array}\right\}+\left[\begin{array}{ccccc}c & 0 & 0 & \cdots & 0 \\ 0 & c_{1} & 0 & \cdots & 0 \\ 0 & 0 & c_{2} & \cdots & 0 \\ \vdots & \vdots & \vdots & \ddots & \vdots \\ 0 & 0 & 0 & \cdots & c_{n}\end{array}\right]\left\{\begin{array}{c}\dot{x} \\ \dot{x}_{1} \\ \dot{x}_{2} \\ \vdots \\ \dot{x}_{n}\end{array}\right\}+\left[\begin{array}{ccccc}k+k_{1}+\cdots k_{n} & -k_{1} & -k_{2} & \cdots & -k_{n} \\ -k_{1} & k_{1} & 0 & \cdots & 0 \\ -k_{2} & 0 & k_{2} & \cdots & 0 \\ \vdots & \vdots & \vdots & \ddots & \vdots \\ -k_{n} & 0 & 0 & \cdots & k_{n}\end{array}\right]\left\{\begin{array}{c}x \\ x_{1} \\ x_{2} \\ \vdots \\ x_{n}\end{array}\right\}=\left\{\begin{array}{c}f \\ 0 \\ 0 \\ \vdots \\ 0\end{array}\right\}$ 
Writing Eq. (4.1) in state-space form,

$$
\left\{\begin{array}{c}
\ddot{x} \\
\dot{x} \\
\dot{x}_{1} \\
\dot{x}_{2} \\
\vdots \\
\dot{x}_{n}
\end{array}\right\}=\left[\begin{array}{cccccc}
-\frac{c}{m} & -\frac{k+k_{1}+\cdots k_{n}}{m} & \frac{k_{1}}{m} & \frac{k_{2}}{m} & \cdots & \frac{k_{n}}{m} \\
1 & 0 & 0 & 0 & \cdots & 0 \\
0 & \frac{1}{\tau_{1}} & -\frac{1}{\tau_{1}} & 0 & \cdots & 0 \\
0 & \frac{1}{\tau_{2}} & 0 & -\frac{1}{\tau_{2}} & \cdots & 0 \\
\vdots & \vdots & \vdots & \vdots & \ddots & \vdots \\
0 & \frac{1}{\tau_{n}} & 0 & 0 & \cdots & -\frac{1}{\tau_{n}}
\end{array}\right]\left\{\begin{array}{c}
\dot{x} \\
x \\
x_{1} \\
x_{2} \\
\vdots \\
x_{n}
\end{array}\right\}+\left\{\begin{array}{c}
f^{\prime} \\
0 \\
0 \\
0 \\
\vdots \\
0
\end{array}\right\}
$$

where $f^{\prime}=f / m, \tau_{i}=c_{i} / k_{i}$ is the time constant of each Maxwell element and $n$ is the number of Maxwell elements in the model. The modal analysis is based on the eigenvalue problem of the $n+2$ by $n+2$ system matrix in Eq. (4.2), which yields $n$ real and one complex conjugate pair of eigenvalues when the system is underdamped. For the purpose of single mass system vibration analysis, only the conjugated eigenvalues are of concern from which the natural frequency $\omega_{n}$ and damping ratio $\zeta$ are determined.

In order for a general Maxwell system, as given in Eq. (4.1) or (4.2), to accurately represent a real physical isolation mount, the parameters of the model must be identified using test data collected from experiments conducted on the mount. For example, the isolated mass $m$ can be measured on a weight scale and the primary linear spring stiffness $k$ can be estimated from a static stiffness experiment. Therefore, $c, c_{i}$ and $k_{i}, i \in[1, n]$ are parameters left to be determined.

\subsection{Identification of General Maxwell Systems Using M-V Models}

The previous method of parameter identification is only suited for M-V models. So the question arises, can a M-V model simulate the dynamic behavior of the general 
Maxwell system which has two or more Maxwell elements? In the case where the parameters of some Maxwell elements in a general Maxwell system satisfy Eq. (4.3),

$$
\tau_{i}=\tau_{e q} \quad(i=1, \cdots, m)
$$

where $m$ is the number of Maxwell elements which satisfy Eq. (4.3), then,

$$
c_{i}=\tau_{i} k_{i}=\tau_{e q} k_{i} \quad(i=1, \cdots, m)
$$

Summing the terms in Eq. (4.4) on both sides,

$$
\sum_{i=1}^{m} c_{i}=\tau_{e q} \sum_{i=1}^{m} k_{i}
$$

Let

$$
k_{e q}=\sum_{i=1}^{m} k_{i}, \quad c_{e q}=\sum_{i=1}^{m} c_{i}
$$

then,

$$
\tau_{e q}=\frac{c_{e q}}{k_{e q}}
$$

The dynamic stiffness of single Maxwell element,

$$
\frac{1}{\frac{1}{k_{i}}+\frac{1}{j \omega c_{i}}}=\frac{j \omega c_{i}}{1+j \omega \frac{c_{i}}{k_{i}}}=\frac{j \omega c_{i}}{1+j \omega \tau_{i}} \quad(i=1, \cdots, m)
$$

The dynamic stiffness of equivalent Maxwell element,

$$
\frac{1}{\frac{1}{k_{e q}}+\frac{1}{j \omega c_{e q}}}=\frac{j \omega c_{e q}}{1+j \omega \frac{c_{e q}}{k_{e q}}}=\frac{j \omega c_{e q}}{1+j \omega \tau_{e q}}
$$

Summing the dynamic stiffness of every single Maxwell element,

$$
\sum_{i=1}^{m} \frac{j \omega c_{i}}{1+j \omega \tau_{i}}=\sum_{i=1}^{m} \frac{j \omega c_{i}}{1+j \omega \tau_{e q}}=\frac{j \omega \sum_{i=1}^{m} c_{i}}{1+j \omega \tau_{e q}}=\frac{j \omega c_{e q}}{1+j \omega \tau_{e q}}
$$


So the equivalent Maxwell element has the same dynamic stiffness as the sum of the dynamic stiffnesses of these Maxwell elements. In this case, the previous parameter identification method can be used for this general Maxwell system. However, what happens if Eq. (4.3) is not satisfied? In order to answer this question, six different general Maxwell systems with two Maxwell elements (M-M-V systems) are considered. All parameters of the six systems are listed in Table 4.1.

There are two Type A Maxwell elements in System A, two Type B Maxwell elements in System B, two Type C Maxwell elements in System C, one Type A and one Type B Maxwell element in System D, one Type A and one Type C Maxwell element in System $E$, and one Type B and one Type $C$ Maxwell element in System $F$. From the frequency response spectra of the actual M-M-V systems, the parameter identification method in chapter 3 identifies these systems as M-V models. The resulting parameters of identified $\mathrm{M}-\mathrm{V}$ models are listed in Table 4.2, and the frequency response spectra of the actual M-M-V systems and identified M-V models are shown in Fig. 4.2. 
Table 4.1. Parameters of Actual M-M-V Systems.

\begin{tabular}{|c|c|c|c|c|c|c|}
\hline Parameters & $M-M-V$ & $M-M-V$ & $M-M-V$ & $M-M-V$ & $M-M-V$ & $M-M-V$ \\
\hline$m(\mathrm{~kg})$ & 1 & 1 & 1 & 1 & 1 & 1 \\
\hline$k(\mathrm{kN} / \mathrm{m})$ & 12 & 12 & 12 & 12 & 12 & 12 \\
\hline$c(\mathrm{~N}-\mathrm{s} / \mathrm{m})$ & 40 & 40 & 40 & 40 & 40 & 40 \\
\hline$k_{1}(\mathrm{kN} / \mathrm{m})$ & 12 & 12 & 12 & 24 & 24 & 12 \\
\hline$c_{1}(\mathrm{~N}-\mathrm{s} / \mathrm{m})$ & 20 & 80 & 2000 & 20 & 20 & 80 \\
\hline$\tau_{1}(\mathrm{~ms})[T y p e]$ & $1.67[A]$ & $6.67[B]$ & $166.67[C]$ & $1.67[A]$ & $1.67[A]$ & $6.67[B]$ \\
\hline$k_{2}(\mathrm{kN} / \mathrm{m})$ & 24 & 24 & 24 & 12 & 12 & 24 \\
\hline$c_{2}(\mathrm{~N}-\mathrm{s} / \mathrm{m})$ & 20 & 120 & 2000 & 80 & 2000 & 2000 \\
\hline$\tau_{2}(\mathrm{~ms})[T y p e]$ & $0.83[A]$ & $5.00[B]$ & $83.33[C]$ & $6.67[B]$ & $166.67[C]$ & $83.33[C]$ \\
\hline$\omega_{\mathrm{n}}(\mathrm{rad} / \mathrm{s})$ & 112.684 & 216.050 & 219.718 & 152.126 & 156.833 & 214.806 \\
\hline$\zeta$ & 0.371 & 0.404 & 0.108 & 0.432 & 0.203 & 0.182 \\
\hline
\end{tabular}


Table 4.2. Parameters of Identified M-V Models.

\begin{tabular}{|c|c|c|c|c|c|c|}
\hline Parameters & $M-V$ & $M-V$ & $M-V$ & $M-V$ & $M-V$ & $M-V$ \\
& Model A & Model B & Model C & Model D & Model E & Model F \\
\hline$c(\mathrm{~N}-\mathrm{s} / \mathrm{m})$ & 49.702 & 40.382 & 39.724 & 58.030 & 60.637 & 61.395 \\
\hline$k_{1}(\mathrm{kN} / \mathrm{m})$ & 18.788 & 35.492 & 35.987 & 12.695 & 12.420 & 33.221 \\
\hline$c_{1}(\mathrm{~N}-\mathrm{s} / \mathrm{m})$ & 30.447 & 196.437 & 3476.033 & 83.162 & 2072.516 & 1482.569 \\
\hline$\tau_{1}(\mathrm{~ms})[T y p e]$ & $1.62[A]$ & $5.53[B]$ & $96.59[C]$ & $6.55[B]$ & $166.87[C]$ & $44.63[C]$ \\
\hline
\end{tabular}



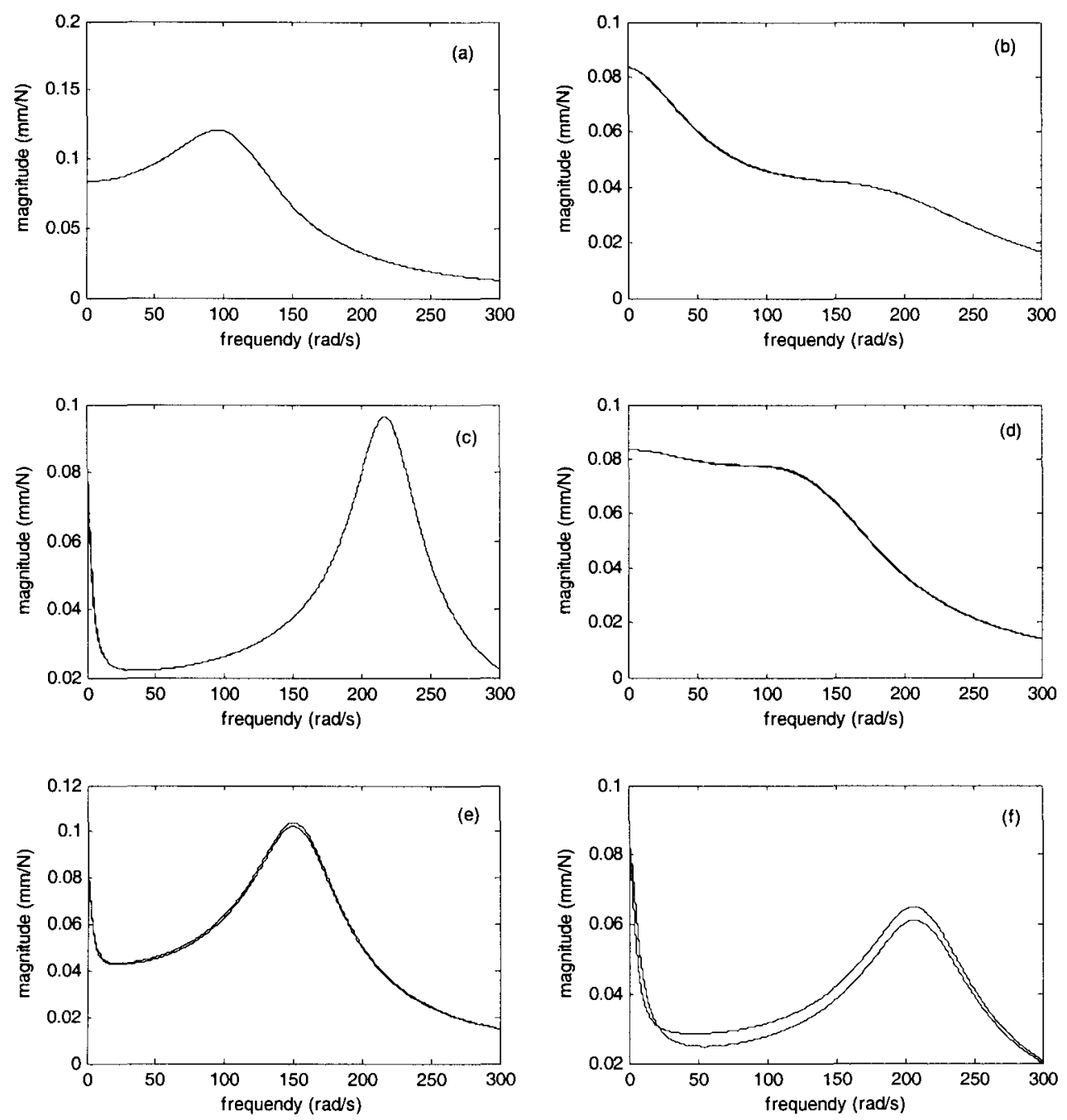

Fig. 4.2. Frequency response of M-M-V Systems and corresponding identified $M-V$ models (a) System and Model A; (b) System and Model B; (c) System and Model C; (d) System and Model D; (e) System and Model E; (f) System and Model F.

As illustrated in Fig. 4.2(a-c), the frequency response curves of the identified M$\mathrm{V}$ models closely match those of the corresponding M-M-V systems. The Maxwell 
element is Type $A$ in identified $M$-V Model $A$, Type $B$ in identified $M-V$ Model $B$ and Type $C$ in identified $M-V$ Model $C$. This illustrates that $\mathrm{M}-\mathrm{V}$ models can be accurately identified for M-M-V systems if the two Maxwell elements making up the systems are of the same Type.

Since the Type $A$ elements in $M-M-V$ System $D$ and $E$ are damping dominant, the effective stiffness of these systems is approximately the sum $c+c_{1}$. Consequently, the identification process identifies the primary dampers of the corresponding $\mathrm{M}-\mathrm{V}$ models as approximately this sum. Also, the Maxwell element of the $M-V$ Model $D$ is identified as Type $B$ and the Maxwell element of the $M-V$ Model $E$ is identified as Type $C$, which are consistent types compared to the non-Type A Maxwell elements making up $M-M-V$ System $D$ and $E$. Figures 4.2 (d-e) comparing the frequency response curves of the identified $M-V$ Model $D$ and $E$ to those of the $M-M-V$ System $D$ and $E$, respectively, illustrate that since damping dominant elements exist in $M-M-V$ System $D$ and $E$, the identified $\mathrm{M}-\mathrm{V}$ models can accurately represent these systems.

Unfortunately, the frequency response spectrum of identified $M$ - $V$ Model $F$ does not accurately match the actual $M-M-V$ System $F$ (Fig. 4.2(f)). Although the Type $C$ element of the $M-M-V$ System $F$ is stiffness dominant, the primary spring stiffness $k$ of the model is fixed since it's value is determined from static stiffness experiment. Also, no single Type B or Type C Maxwell element can simulate the combined behavior of a Type $B$ and Type $C$ element. Consequently, a parameter identification method for Maxwell models having two or more Maxwell elements is necessary. 


\subsection{Parameter Identification Using Constraint Optimization}

The parameter identification process for determining the $2 n+1$ unknowns $c, c_{1}, \ldots$, $c_{\mathrm{n}}$ and $k_{1}, \ldots, k_{\mathrm{n}}$ of Maxwell models with $n$ Maxwell elements is based on the nonlinear constraint optimization problem:

$$
f(\kappa)=\sum_{j=1}^{N} a b s\left(H_{j}(\kappa)-H_{j}\right) \rightarrow \min \quad \text { for } \kappa \in \Omega^{2 n+1}
$$

where $N$ is the number of discrete frequencies of the measured complex frequency response $H_{j}$. The vector $\kappa$ contains the $2 n+1$ unknown parameters of the general Maxwell model governed by Eq. (4.1) and $H_{j}(\kappa)$ are calculated complex frequency response data of the model at the discrete frequencies. The optimization problem Eq. (4.11) minimizes the objective function $f(\kappa)$, where the parameters $\kappa$ are subjected to the following constraints:

$$
\left(\omega_{n}(\kappa)-\omega_{n}\right)^{2}=0 \quad \text { and } \quad(\zeta(\kappa)-\zeta)^{2}=0
$$

where $\omega_{n}(\kappa)$ and $\zeta(\kappa)$ are the calculated natural frequency and damping ratio governed by Eq. (4.2), and $\omega_{n}$ and $\zeta$ are estimated from the measured frequency response. A sequential quadratic programming (SQP) method is used as the solution algorithm for the identification [41].

\subsubsection{Parameter identification of $M-V$ systems}

To illustrate the effectiveness of parameter identification using the optimization method, the three numeric examples listed in Table 3.3, M-V systems, are investigated first. To simulate the measured frequency response functions in Eq. (4.11), random noise is included in the calculated frequency response functions

$$
H_{j}=H_{j}\left(\kappa_{0}\right)+\alpha_{j} H_{j}\left(\kappa_{0}\right)
$$


where $\alpha_{\mathrm{j}}$ is a normally distributed variable with zero mean and standard deviation $\sigma=0$, 0.01 and 0.05 . The vector $\kappa_{0}$ consists of the parameters of original M-V systems. The frequency $\omega_{\mathrm{j}}$ ranges form 0 to $300 \mathrm{rad} / \mathrm{s}$ and 300 evenly distributed data points are used for identification. Because the mass and primary stiffness are known, only three parameters need to be identified. The identified results are listed in Table 4.3.

Table 4.3. Identified parameters of M-V models using optimization method.

\begin{tabular}{|c|c|c|c|c|c|c|c|c|c|}
\hline Parameter & \multicolumn{3}{|c|}{ M-V Model A } & \multicolumn{3}{c|}{ M-V Model B } & \multicolumn{3}{c|}{ M-V Model C } \\
\hline$\sigma$ & 0 & 0.01 & 0.05 & 0 & 0.01 & 0.05 & 0 & 0.01 & 0.05 \\
\hline$c(\mathrm{~N}-\mathrm{s} / \mathrm{m})$ & 40.00 & 35.41 & 32.10 & 40.00 & 39.70 & 38.22 & 40.01 & 39.99 & 40.74 \\
\hline$k_{1}(\mathrm{kN} / \mathrm{m})$ & 12.00 & 17.80 & 22.72 & 12.00 & 12.06 & 12.36 & 12.00 & 12.00 & 12.00 \\
\hline$c_{1}(\mathrm{~N}-\mathrm{s} / \mathrm{m})$ & 20.00 & 24.45 & 27.69 & 80.00 & 80.02 & 80.16 & 400.0 & 399.4 & 419.9 \\
\hline
\end{tabular}

In the case where $\sigma=0$, the identified M-V models are almost the same as the parameters of original M-V systems. In the case where experimental error exists, the identified results of M-V Model C only differ slightly from the actual values, but the identified results of M-V Model A differ considerably. Recall the results shown in Fig. 3.14-3.16. In the case where the Maxwell element in the M-V model is Type A, the frequency response spectrum changes only a little as the parameters change. In the case where the Maxwell element in the M-V model is Type $C$, the frequency response spectrum changes a lot as the parameters change. Therefore, the parameters of identified M-V Model A change a lot when experimental error exists, but the parameters of identified M-V Model $C$ change only a little when experimental error exists. Although the 
identified parameters are not exactly the same as the parameters of the original $\mathrm{M}-\mathrm{V}$ systems when experimental error exists, the frequency response spectrum of identified $\mathrm{M}-\mathrm{V}$ models are almost the same as those of original M-V systems. Figure 4.3 shows the frequency response spectrum of identified M-V models (for $\sigma=0.05$ ) and the original MV systems. 

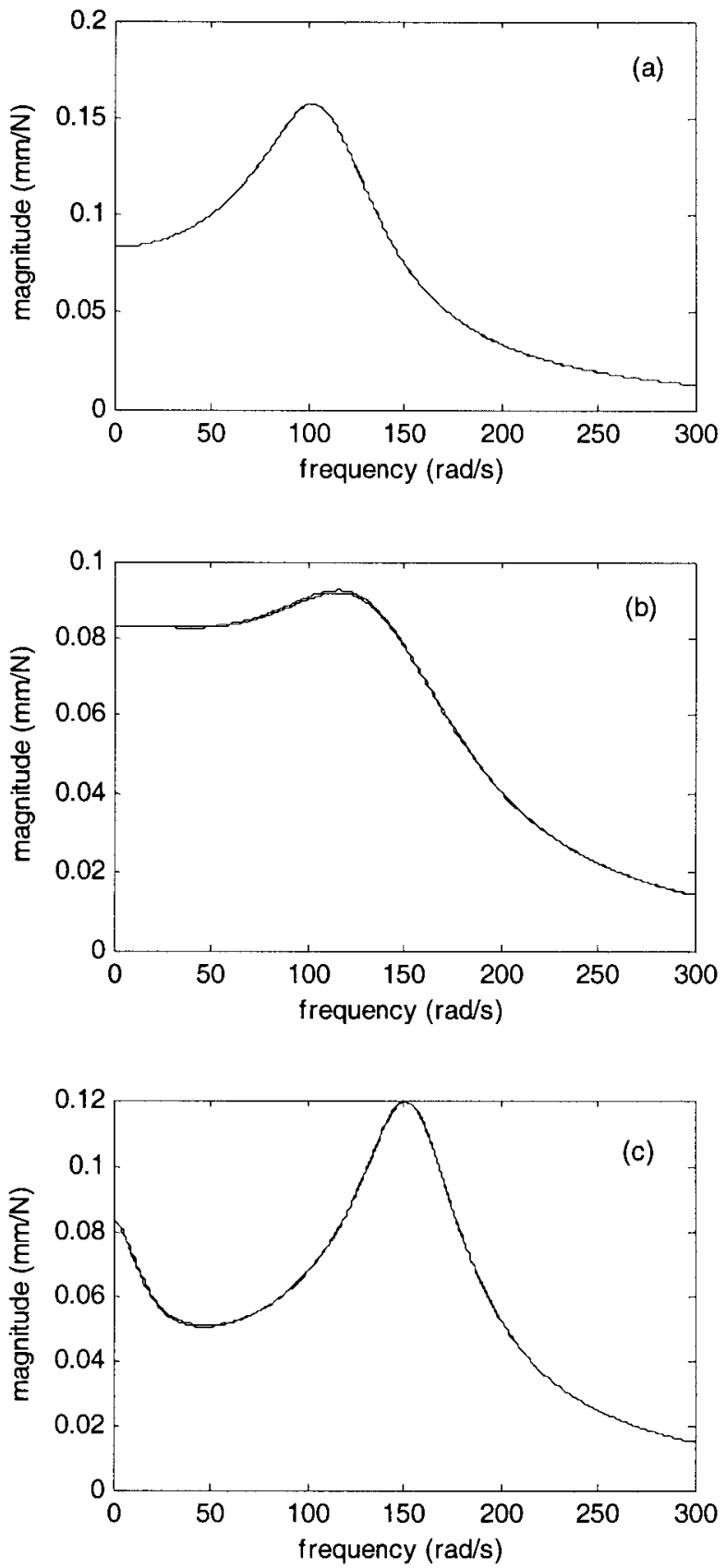

Fig. 4.3. Frequency response functions of (a) M-V System $A$ and M-V Model A ( $\sigma=$ 0.05); (b) M-V System $B$ and M-V Model $B(\sigma=0.05)$; (c) M-V System $C$ and M-V Model $C(\sigma=0.05)$. 


\subsubsection{Parameter identification of $M-M-V$ systems}

The parameters of the identified $M-M-V$ Model $F$ are listed in Table 4.4, which match closely to the parameters of actual $M-M-V$ System $F$ in the case of $\sigma=0$ and 0.01 . Although the results are different in case of $\sigma=0.05$, the frequency response spectrum of identified $M-M-V$ Model $F$ is very close to that of actual M-M-V System $F$ (Fig. 4.4).

Table 4.4. Parameters of Identified M-M-V Model F.

\begin{tabular}{|c|c|c|c|}
\hline$\sigma$ & 0 & 0.01 & 0.05 \\
\hline$c(\mathrm{~N}-\mathrm{s} / \mathrm{m})$ & 39.9903 & 39.9552 & 43.5938 \\
\hline$k_{1}(\mathrm{kN} / \mathrm{m})$ & 12.0025 & 11.9991 & 11.3901 \\
\hline$c_{1}(\mathrm{~N}-\mathrm{s} / \mathrm{m})$ & 79.9205 & 79.7038 & 86.9666 \\
\hline$k_{2}(\mathrm{kN} / \mathrm{m})$ & 24.0041 & 24.0201 & 23.8265 \\
\hline$c_{2}(\mathrm{~N}-\mathrm{s} / \mathrm{m})$ & 1999.97 & 1998.76 & 1993.82 \\
\hline
\end{tabular}




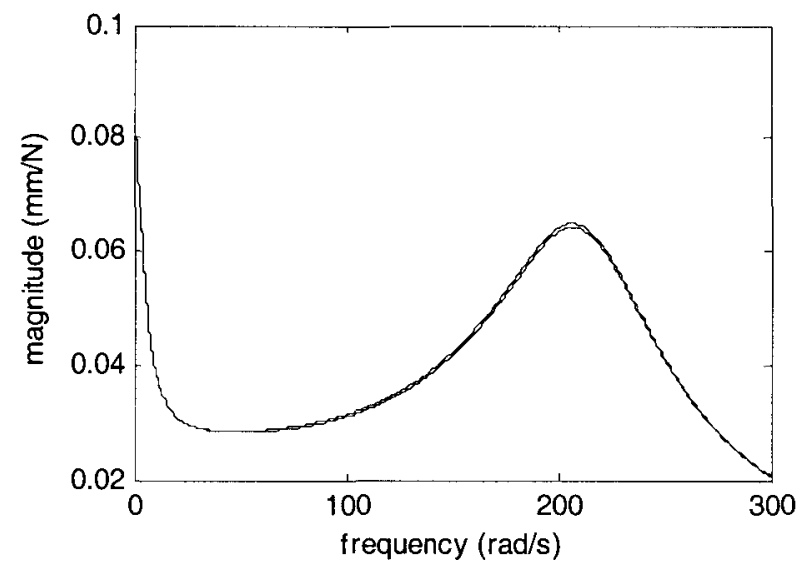

Fig 4.4. Frequency response spectra of actual M-M-V System $F$ and identified M-M-V Model $F(\sigma=0.05)$

Now consider two general Maxwell systems in Table 4.5. There are three Maxwell elements in the general Maxwell System A and six Maxwell elements in general Maxwell System $B$. The optimization method is used for identifying these Maxwell systems as M-M-V models (i.e., having only 2 Maxwell elements). The resulting identified parameters of the models are listed in Table 4.6. The frequency response spectra of identified M-M-V models match the frequency response spectra of actual general Maxwell systems very well (Fig. 4.5). The identified models are not unique since the identified parameters vary depending on the initial guesses chosen. However, accurate estimates of the frequency response can be achieved if the following two rules are utilized when selecting the initial guesses. First, the initial values for the elements of the Maxwell model should be chosen so that the resulting element Types are not the same, e.g., choose one element to be Type $B$ and one Type $C$. Second, the initial guesses of the elements should be chosen so that the natural frequency and damping ratio of the Maxwell model are close to that of the system. Curves of the influences of $c_{i}$ and $k_{i}$ on 
natural frequency and damping ratio from reference are helpful in choosing initial guesses. Although it is difficult to know exactly how many Maxwell elements are necessary to identify an actual Maxwell system, these examples show that it is possible to identify a Maxwell model with only two Maxwell elements that can accurately simulate the static and dynamic behavior of a Maxwell system having more Maxwell elements.

From the identified results, it is concluded that the two Maxwell elements in identified M-M-V model which can accurately simulate the static and dynamic behavior of Maxwell system should belong to Type $B$ and Type $C$. Because the Type A element is damping dominant, the combination of Type A element and the primary damper can be replaced by a new damper without significant modeling error. The Type $C$ element is stiffness dominant and the primary spring is fixed by the static stiffness. The combined effect of them can not be replaced by a single spring. The Type B element is neither damping dominant nor stiffness dominant, it play a special effect in the model and can not be replaced by a damper or spring. 
Table 4.5. Parameters of General Maxwell Systems.

\begin{tabular}{|c|c|c|c|c|c|}
\hline \multicolumn{2}{|c|}{ General Maxwell System A } & \multicolumn{5}{|c|}{ General Maxwell System B } \\
\hline$m(\mathrm{~kg})$ & 1 & $m(\mathrm{~kg})$ & 1 & $k_{4}(\mathrm{kN} / \mathrm{m})$ & 24 \\
\hline$k(\mathrm{kN} / \mathrm{m})$ & 12 & $k(\mathrm{kN} / \mathrm{m})$ & 12 & $c_{4}(\mathrm{~N}-\mathrm{s} / \mathrm{m})$ & 120 \\
\hline$c(\mathrm{~N}-\mathrm{s} / \mathrm{m})$ & 40 & $c(\mathrm{~N}-\mathrm{s} / \mathrm{m})$ & 40 & $\tau_{4}(\mathrm{~ms})[T y p e]$ & $5.00[B]$ \\
\hline$k_{1}(\mathrm{kN} / \mathrm{m})$ & 12 & $k_{1}(\mathrm{kN} / \mathrm{m})$ & 12 & $k_{5}(\mathrm{kN} / \mathrm{m})$ & 12 \\
\hline$c_{1}(\mathrm{~N}-\mathrm{s} / \mathrm{m})$ & 20 & $c_{1}(\mathrm{~N}-\mathrm{s} / \mathrm{m})$ & 20 & $c_{5}(\mathrm{~N}-\mathrm{s} / \mathrm{m})$ & 2000 \\
\hline$\tau_{1}(\mathrm{~ms})[T y p e]$ & $1.67[A]$ & $\tau_{1}(\mathrm{~ms})[T y p e]$ & $1.67[A]$ & $\tau_{5}(\mathrm{~ms})[T y p e]$ & $166.67[C]$ \\
\hline$k_{2}(\mathrm{kN} / \mathrm{m})$ & 12 & $k_{2}(\mathrm{kN} / \mathrm{m})$ & 24 & $k_{6}(\mathrm{kN} / \mathrm{m})$ & 24 \\
\hline$c_{2}(\mathrm{~N}-\mathrm{s} / \mathrm{m})$ & 80 & $c_{2}(\mathrm{~N}-\mathrm{s} / \mathrm{m})$ & 20 & $c_{6}(\mathrm{~N}-\mathrm{s} / \mathrm{m})$ & 2000 \\
\hline$\tau_{2}(\mathrm{~ms})[T y p e]$ & $6.67[B]$ & $\tau_{2}(\mathrm{~ms})[T y p e]$ & $0.83[A]$ & $\tau_{6}(\mathrm{~ms})[T y p e]$ & $83.33[C]$ \\
\hline$k_{3}(\mathrm{kN} / \mathrm{m})$ & 24 & $k_{3}(\mathrm{kN} / \mathrm{m})$ & 12 & $\omega_{\mathrm{n}}(\mathrm{rad} / \mathrm{s})$ & 293.87 \\
\hline$c_{3}(\mathrm{~N}-\mathrm{s} / \mathrm{m})$ & 2000 & $c_{3}(\mathrm{~N}-\mathrm{s} / \mathrm{m})$ & 80 & $\zeta$ & 0.26 \\
\hline$\tau_{3}(\mathrm{~ms})[$ Type $]$ & $83.33[C]$ & $\tau_{3}(\mathrm{~ms})[$ Type $]$ & $6.67[B]$ & - & - \\
\hline$\omega_{\mathrm{n}}(\mathrm{rad} / \mathrm{s})$ & 112.68 & - & - & - & - \\
\hline$\zeta$ & 0.37 & - & - & - & - \\
\hline & & & & & \\
\hline
\end{tabular}


Table 4.6. Parameters of identified general Maxwell models.

\begin{tabular}{|c|c|c|c|}
\hline \multicolumn{2}{|c|}{ General Maxwell Model $A$} & \multicolumn{2}{|c|}{ General Maxwell Model B } \\
\hline$c(\mathrm{~N}-\mathrm{s} / \mathrm{m})$ & 49.54 & $c(\mathrm{~N}-\mathrm{s} / \mathrm{m})$ & 65.73 \\
\hline$k_{1}(\mathrm{kN} / \mathrm{m})$ & 14.95 & $k_{1}(\mathrm{kN} / \mathrm{m})$ & 40.69 \\
\hline$c_{1}(\mathrm{~N}-\mathrm{s} / \mathrm{m})$ & 81.76 & $c_{1}(\mathrm{~N}-\mathrm{s} / \mathrm{m})$ & 209.15 \\
\hline$\tau_{1}(\mathrm{~ms})[$ Type $]$ & $5.47[B]$ & $\tau_{1}(\mathrm{~ms})[$ Type $]$ & $5.14[B]$ \\
\hline$k_{2}(\mathrm{kN} / \mathrm{m})$ & 24.65 & $k_{2}(\mathrm{kN} / \mathrm{m})$ & 36.50 \\
\hline$c_{2}(\mathrm{~N}-\mathrm{s} / \mathrm{m})$ & 2003.24 & $c_{2}(\mathrm{~N}-\mathrm{s} / \mathrm{m})$ & 3797.60 \\
\hline$\tau_{2}(\mathrm{~ms})[T y p e]$ & $81.27[C]$ & $\tau_{2}(\mathrm{~ms})[T y p e]$ & $104.05[C]$ \\
\hline
\end{tabular}



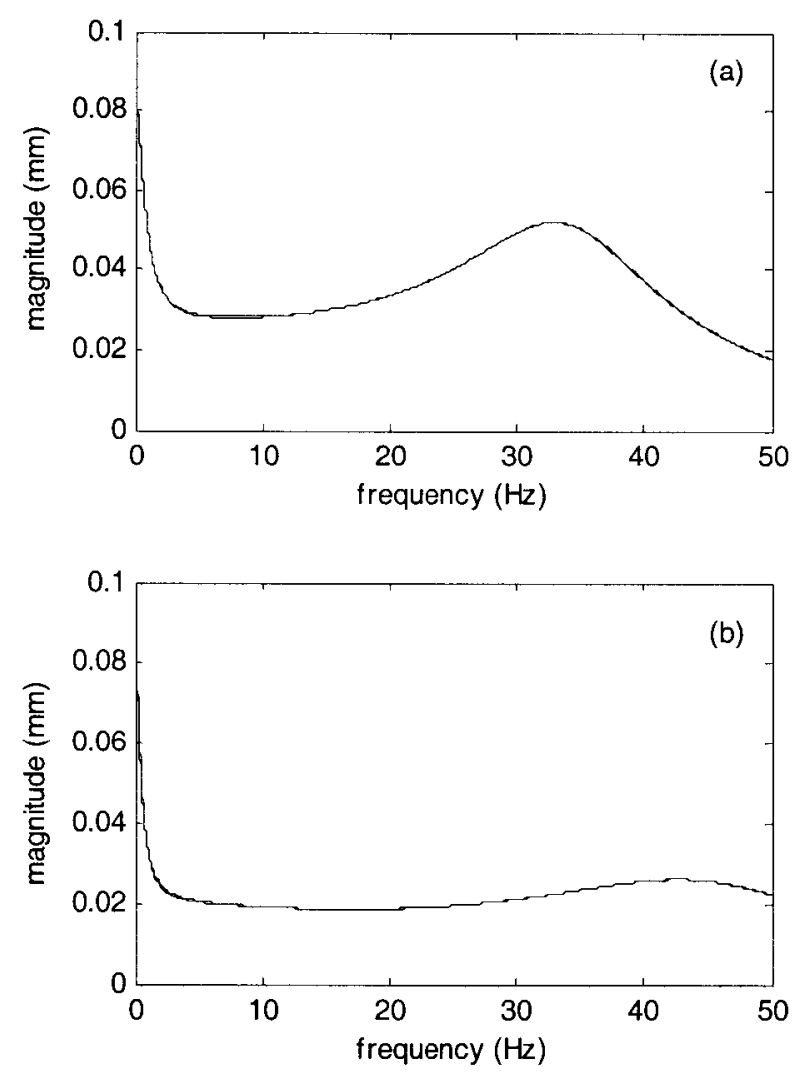

Fig. 4.5. The frequency response spectra of (a) general Maxwell System $A$ and identified M-M-V Model A; (b) general Maxwell System B and identified M-M-V Model B.

\subsection{Summary}

In this chapter, a parameter identification method based on constraint optimization is developed for general Maxwell models that have two or more Maxwell elements in a single mass isolation system by fitting the models to measured frequency response spectra. The effectiveness of the identification method is verified by several analytical examples. These studies reveal that a Maxwell model having only one Maxwell element can simulate the dynamic characteristics of a Maxwell system having 
two Maxwell elements as long as one is not Type $B$ and the other is not Type $C$. These analytical studies also conclude that a Maxwell model having two Maxwell elements (One Type B and one Type $C$ ) can simulate the dynamic characteristics of a Maxwell system having more than two Maxwell elements. 


\section{CHAPTER 5}

\section{PARAMETER IDENTIFICATION OF RUBBER ISOLATORS}

A bubble mount (Fig. 5.1(a)), plate mount (Fig. 5.1(b)) and rubber stud (Fig. 5.1(c)) are considered for the experimental portion of this study. In practice, these mounts are used to isolate vibration and shock in electronic or medical equipment, avionics, computers, small pumps, compressors, appliances, office machines and transportation equipment [42].

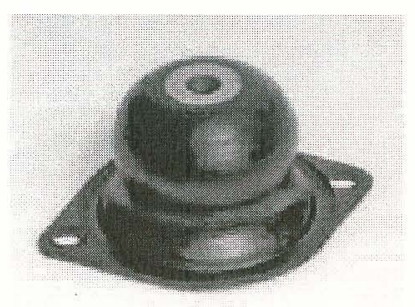

(a)

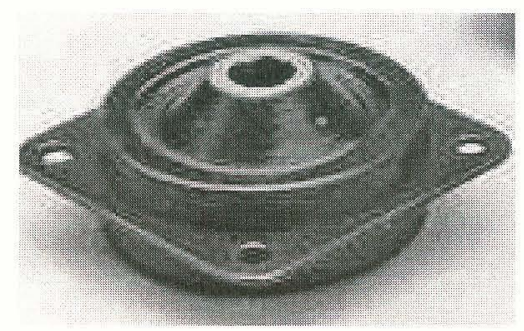

(b)

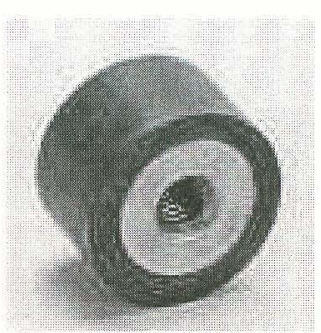

(c)

Fig. 5.1. (a) Bubble mount; (b) plate mount; (c) rubber stud.

\subsection{Static Stiffness Experimental Setup and Results}

The static stiffness experiment employed to determine the primary stiffness $k$ of the isolators is shown in Fig. 5.2 where P-3500 portable strain Indicator and a 3167-50 load cell were used to make enasurements. The deflection is manually adjusted in 0.25 
$\mathrm{mm}$ increments for the bubble mount, $0.1 \mathrm{~mm}$ increments for the plate mount and 0.05 $\mathrm{mm}$ increments for the rubber stud. The forces acting on load cell (connected in series with the mounts) are read after the deflections are fixed for one minute. Both the loading and unloading processes are measured and the resulting load versus deflection curves along with the directions of both loading and unloading are shown Fig. 5.3. The bubble mount exhibits a softening spring behavior for small deflections, but exhibits a hardening spring behavior for large deflections. The plate mount behaves approximately linear and the rubber stud exhibits a hardening spring behavior over the deflection ranges considered. The mass used in frequency response experiment (Chapter 5.2) compresses each mount to a static equilibrium position $x_{o} \approx 0.75,0.5$ and $0.2 \mathrm{~mm}$ for the bubble mount, plate mount and rubber stud, respectively. An average static stiffness for each mount about $x_{o}$ under the loading and unloading processes is taken within the limits of the frequency response experiment by the least means square method. The static stiffness results are listed in Table 5.1.

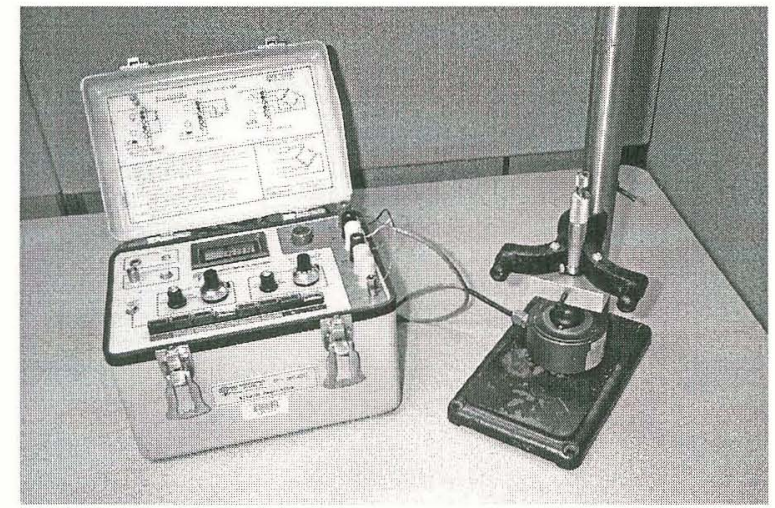

(a)

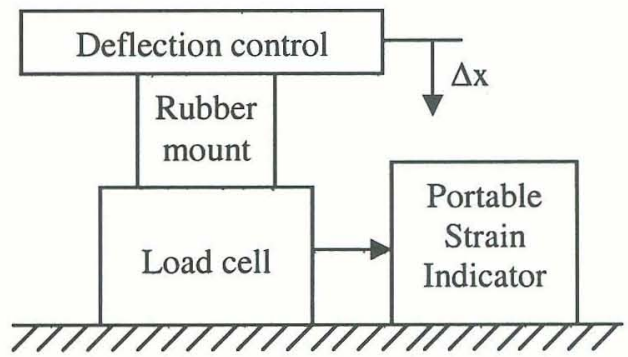

(b)

Fig. 5.2. Static stiffness experiment system. 

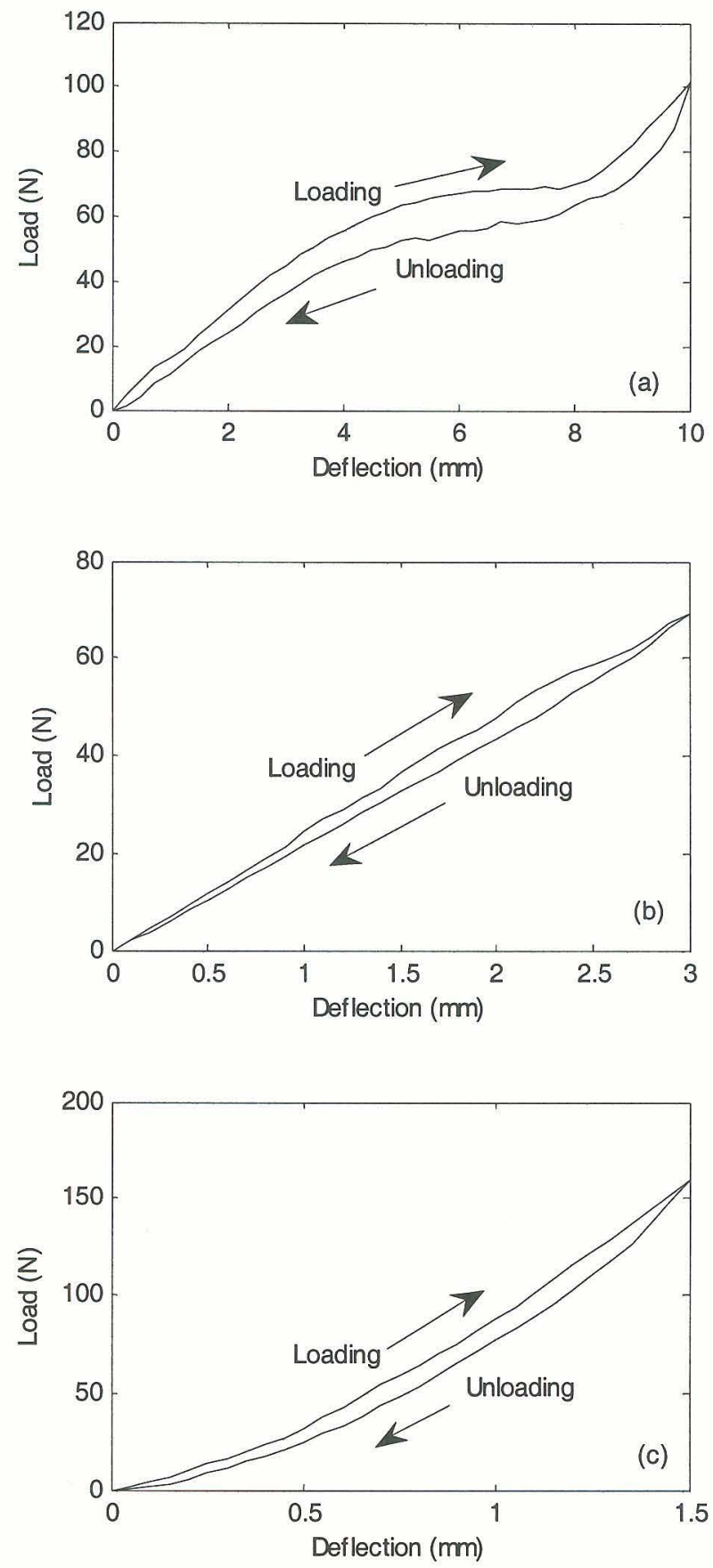

Fig. 5.3. Axial loads vs. deflections of (a) bubble mount; (b) plate mount; (c) rubber stud. 
Table 5.1. Static stiffness of rubble mounts.

\begin{tabular}{|c|c|c|c|}
\hline Mount Type & Bubble mount & Plate mount & Rubber stud \\
\hline Linear Static Stiffness $(\mathrm{kN} / \mathrm{m})$ & 14.013 & 23.151 & 54.507 \\
\hline
\end{tabular}

\subsection{Frequency Response Experimental Setup and Results}

Figure 5.4 contains a photograph and schematic of the experimental setup for measuring frequency response of each isolator mounted to a cylindrical block of mass $m$ $=1.041 \mathrm{~kg}$. A drill press is used to provide a rigid foundation for the experiment, as shown in Figure 5.4(a). To maintain a balanced mass and minimize rocking motion, three accelerometers are used. The average of three frequency spectra are used as experiment results for parameter identification.

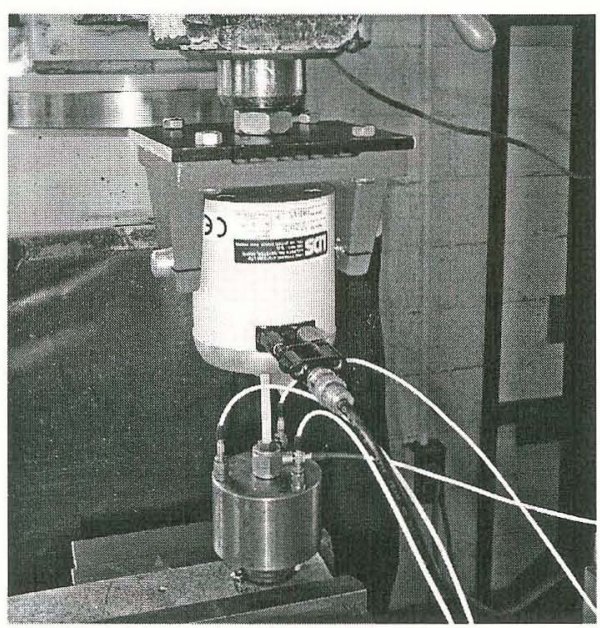

(a)

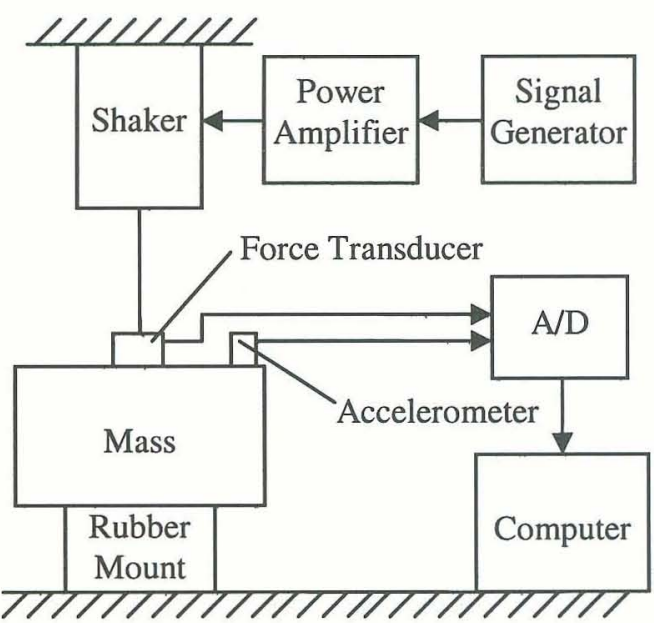

(b)

Fig. 5.4 Dynamic experiment system. 
The experimental setup includes an Agilent 33220A signal generator, a Peavey PMA70+ power amplifier, a LDS V203 shaker, a PCB 208 force transducer, 3 PCB 353 accelerometers, and a National Instruments SCXI 1531 A/D converter with anti-aliasing filters. National Instruments was used for data acquisition. The measured frequency response spectra are estimated via the transfer function of the system with the input forces from the shaker and the output acceleration from the accelerometers using Welch's averaged periodogram method. The transfer function is the quotient of cross power spectral density of input force and output acceleration and the power spectral density of output acceleration. In order to achieve accurate damping property of the system, no window is employed for the calculation. The number of FFT points used to calculation is 10,000 and the sampling frequency is $5,000 \mathrm{~Hz}$. The measured results are shown in Fig. 5.5 result from frequency domain average response spectra of the three accelerometers. Fixed frequency sine excitations are applied in $0.5 \mathrm{~Hz}$ increments from 3 to $80 \mathrm{~Hz}$ for the bubble and plate mount and from 3 to $160 \mathrm{~Hz}$ for the rubber stud. The natural frequencies are determined from zero crossings of the real part of measured frequency response spectra and the damping ratios are estimated by half-power point method. Results are listed in Table 5.2. 

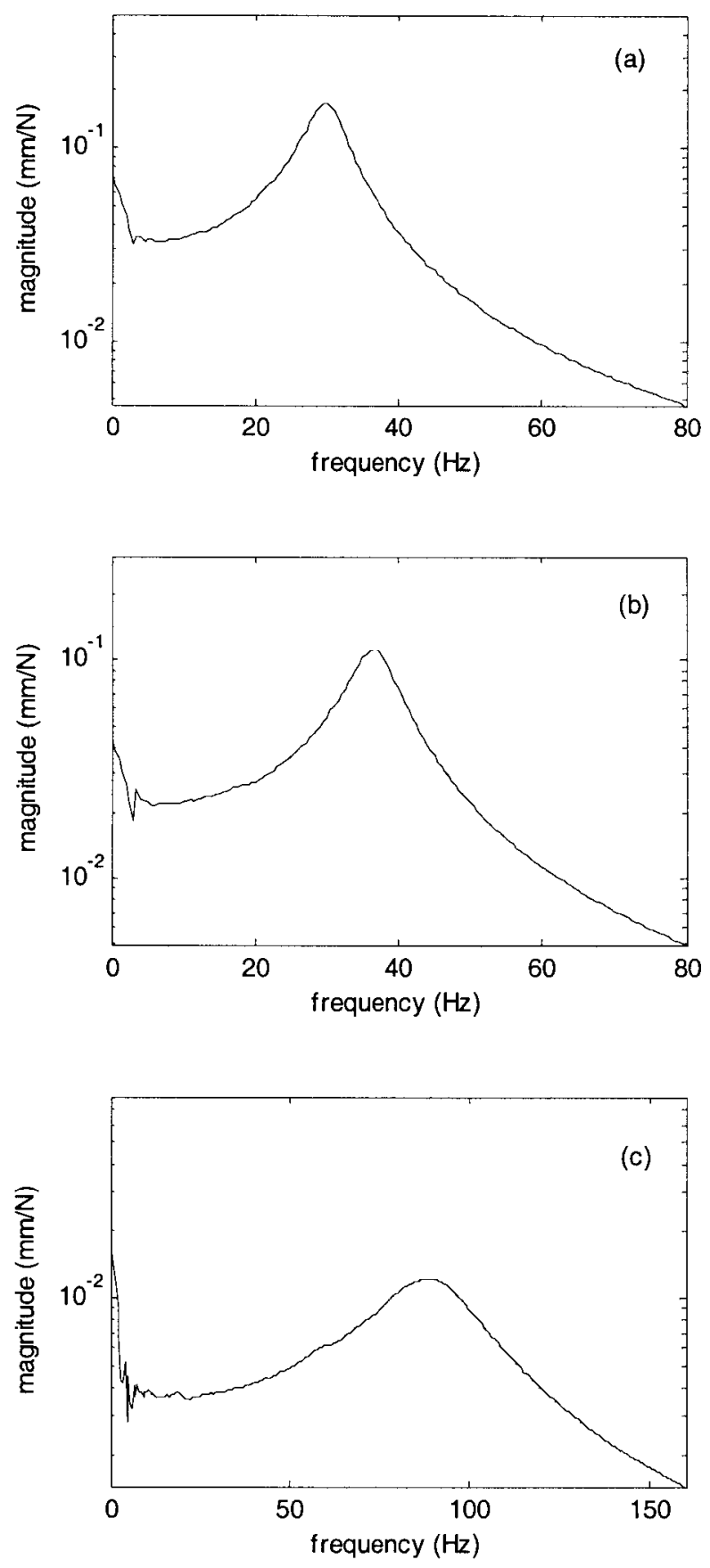

Fig. 5.5. Frequency response spectra of (a) bubble mount; (b) plate mount; (c) rubber stud. 
Table 5.2. Natural frequency and damping ratio of rubble mounts.

\begin{tabular}{|c|c|c|c|}
\hline Part number & Bubble mount & Plate mount & Rubber stud \\
\hline Natural frequency (rad/s) & 187.15 & 229.59 & 559.29 \\
\hline Damping ratio & 0.09 & 0.09 & 0.15 \\
\hline
\end{tabular}

\subsection{Parameter Identification}

The parameter identification methods developed in chapter 3 and 4 will be used to identify the rubber isolators as Voigt, $\mathrm{M}-\mathrm{V}$ and $\mathrm{M}-\mathrm{M}-\mathrm{V}$ models. The accuracy of each model will be illustrated by comparing the frequency responses of each identified model with the corresponding measured frequency responses from experiment.

\subsubsection{Voigt model parameter identification}

Since $\omega_{n}$ and $\zeta$ are estimated from the measured frequency response spectra, two approaches exist to identify the parameters $\mathrm{m}, \mathrm{c}$ and $\mathrm{k}$ of the Voigt models. The models can be identified by using the primary stiffness $k$ determined from the static stiffness experiment, then $m=k / \omega_{n}{ }^{2}$ and $c=2 m \zeta \omega_{n}$. Or, the isolated mass $m$ measured on a weight scale can be used, then $k=m \omega_{n}{ }^{2}$ and $c=2 m \zeta \omega_{n}$. A Voigt model identified by the first approach will be referred to as Voigt Model $K$ - the stiffness consistent Voigt model, and the Voigt model identified by the second approach will be referred to as Voigt Model Mthe mass consistent Voigt model. The corresponding parameters of the identified Voigt models for each rubber mount are listed in Table 5.3. The frequency response of Voigt 
model $K$, Voigt Model $M$ and experiment are show in Fig. 5.6. The frequency response of each Voigt Model $K$ are larger in magnitude than the measured frequency response, while the frequency response of each Voigt Model $M$ are smaller in magnitude than the measured frequency response. The frequency response of each Voigt Model M more closely match the experimentally measured frequency response except in the low frequency range. This is due to the fact that the Voigt Model $M$ can match the natural frequency and damping ratio but not the static stiffness. Alternatively, the Voigt Model K can match the static stiffness, natural frequency and damping ratio. However, the frequency response is shifted higher in magnitude than the measured frequency response across the entire frequency range.

Table 5.3. Parameters of identified Voigt models.

\begin{tabular}{|c|c|c|c|c|}
\hline \multicolumn{2}{|c|}{ Part number } & Bubble mount & Plate mount & Rubber stud \\
\hline \multirow{4}{*}{ Voigt Model K } & $m(\mathrm{~kg})$ & 0.40 & 0.44 & 0.17 \\
\cline { 2 - 5 } & $k(\mathrm{kN} / \mathrm{m})$ & 14.01 & 23.15 & 54.51 \\
\cline { 2 - 5 } & $c(\mathrm{~N}-\mathrm{s} / \mathrm{m})$ & 13.37 & 18.07 & 29.53 \\
\hline \multirow{3}{*}{ Voigt Model M } & $m(\mathrm{~kg})$ & 1.04 & 1.04 & 1.04 \\
\cline { 2 - 5 } & $k(\mathrm{kN} / \mathrm{m})$ & 36.46 & 54.87 & 325.63 \\
\cline { 2 - 5 } & $c(\mathrm{~N}-\mathrm{s} / \mathrm{m})$ & 34.80 & 42.83 & 176.41 \\
\hline
\end{tabular}



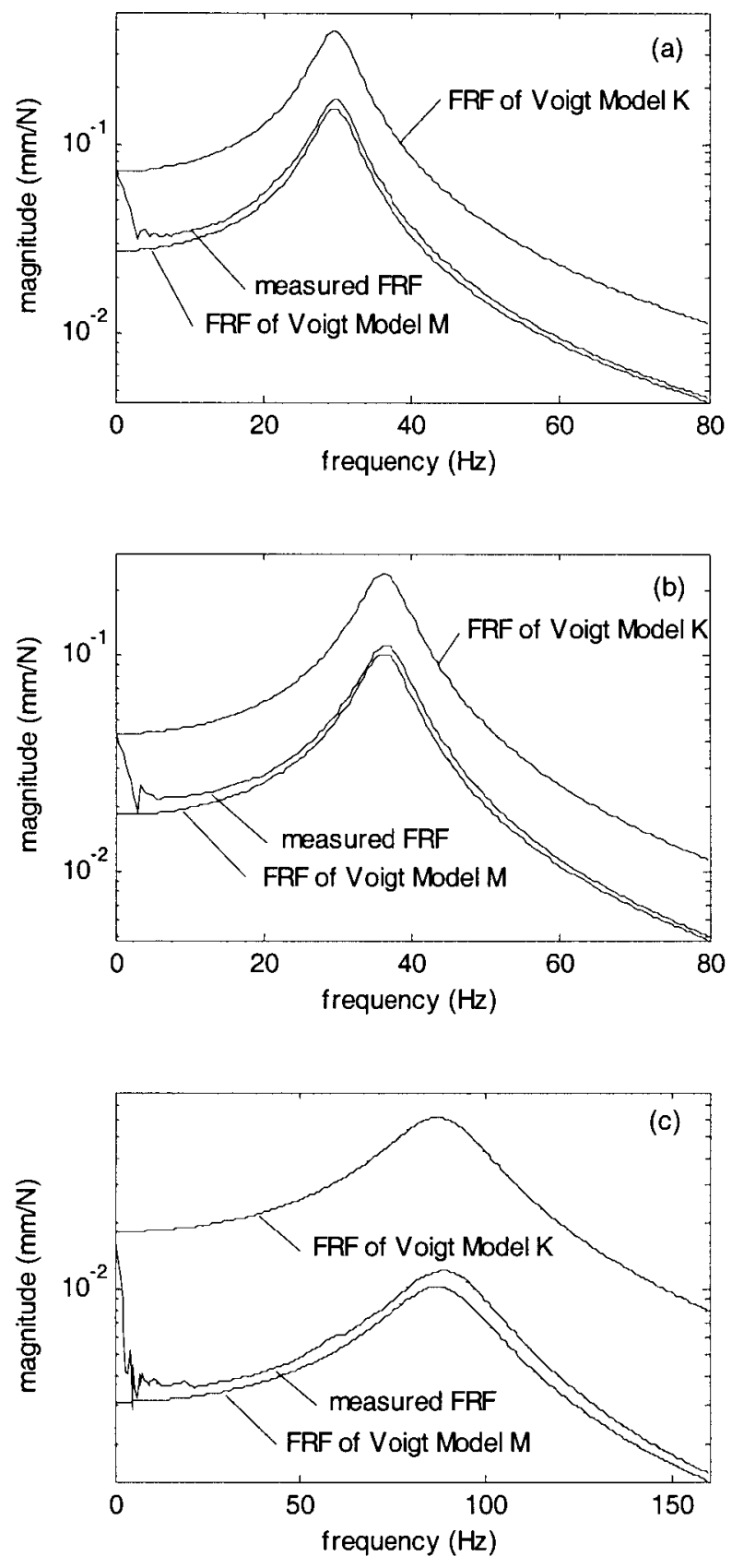

Fig. 5.6. Frequency response spectra of (a) bubble mount; (b) plate mount; (c) rubber stud. 


\subsubsection{Parameter identification as Maxwell-Voigt model}

In this section the method developed in Chapter 3 is utilized to identify M-V models for the three rubber isolators. The identified parameters of the $\mathrm{M}-\mathrm{V}$ models are listed in Table 5.4 (Trial 1) where all the Maxwell elements are identified as Type $C$ stiffness dominant. Consequently, since the models' values for $k$ are chosen to match the experimentally measured static stiffness values, the models' identified values for $k_{1}$ result in sums $k+k_{1}$ equal to values such that the models' natural frequencies match the estimated natural frequencies from experiment. Also, the identified damping coefficients $c$ equal values such that the models' damping ratios match the estimated damping ratios from experiment. Therefore, the M-V models match not only natural frequency and damping ratio but also static stiffness. The measured and identified frequency responses are show in Fig. 5.7. The frequency response of M-V Models match much closer to the experimentally measured frequency response compared to those of the respective Voigt Model $M$ in the low frequency range. However, the accuracy of the M-V Models' frequency responses are similar to those of the respective Voigt Model $M$ near and above the peak.

From Chapter 3, it is shown that the M-V Model's frequency response will increase in magnitude as the value of primary damper $c$ decreases. Therefore, in order to closer match the models' frequency response with experiment in the range near the natural frequency, another group of parameters are identified as listed in Table 5.5 (Trial 2). Here the identified Maxwell elements belong to Type $B$ and the primary damping coefficients $c$ are negative. Since the Type $B$ elements contribute damping to the system, the combined dynamic effect of the Type B element and primary damper are similar to the primary 
damper of M-V models in Table 5.4 (Trial 1). The Type B elements also contribute stiffness to the system with a dynamic effect similar to the Type C elements of M-V models in Table 5.4 (Trial 1). The frequency responses of these new M-V Models are illustrated in Fig. 5.8. As shown, the curves match very well in the range near the natural frequency, but not well in the low frequency range.

Table 5.4. Parameters of identified Maxwell-Voigt models (Trial 1).

\begin{tabular}{|c|c|c|c|}
\hline Isolator Type & Bubble mount & Plate mount & Rubber stud \\
\hline$m(\mathrm{~kg})$ & 1.041 & 1.04 & 1.04 \\
\hline$k(\mathrm{kN} / \mathrm{m})$ & 14.01 & 23.15 & 54.51 \\
\hline$c(\mathrm{~N}-\mathrm{s} / \mathrm{m})$ & 25.81 & 31.877 & 153.36 \\
\hline$k_{1}(\mathrm{kN} / \mathrm{m})$ & 22.27 & 31.47 & 267.83 \\
\hline$c_{1}(\mathrm{~N}-\mathrm{s} / \mathrm{m})$ & 1588.57 & 1729.11 & 10070.89 \\
\hline$\tau_{1}(\mathrm{~ms})[T y p e]$ & $71.32[C]$ & $55.09[C]$ & $37.60[C]$ \\
\hline
\end{tabular}

Table 5.5. Parameters of identified Maxwell-Voigt models (Trial 2).

\begin{tabular}{|c|c|c|c|}
\hline Isolator Type & Bubble mount & Plate mount & Rubber stud \\
\hline$m(\mathrm{~kg})$ & 1.04 & 1.04 & 1.04 \\
\hline$k(\mathrm{kN} / \mathrm{m})$ & 14.01 & 23.15 & 54.51 \\
\hline$c(\mathrm{~N}-\mathrm{s} / \mathrm{m})$ & -40 & -27 & -125 \\
\hline$k_{1}(\mathrm{kN} / \mathrm{m})$ & 28.68 & 36.95 & 324.86 \\
\hline$c_{1}(\mathrm{~N}-\mathrm{s} / \mathrm{m})$ & 245.73 & 318.44 & 934.16 \\
\hline$\tau_{1}(\mathrm{~ms})[$ Type $]$ & $8.57[B]$ & $8.62[B]$ & $2.88[B]$ \\
\hline
\end{tabular}



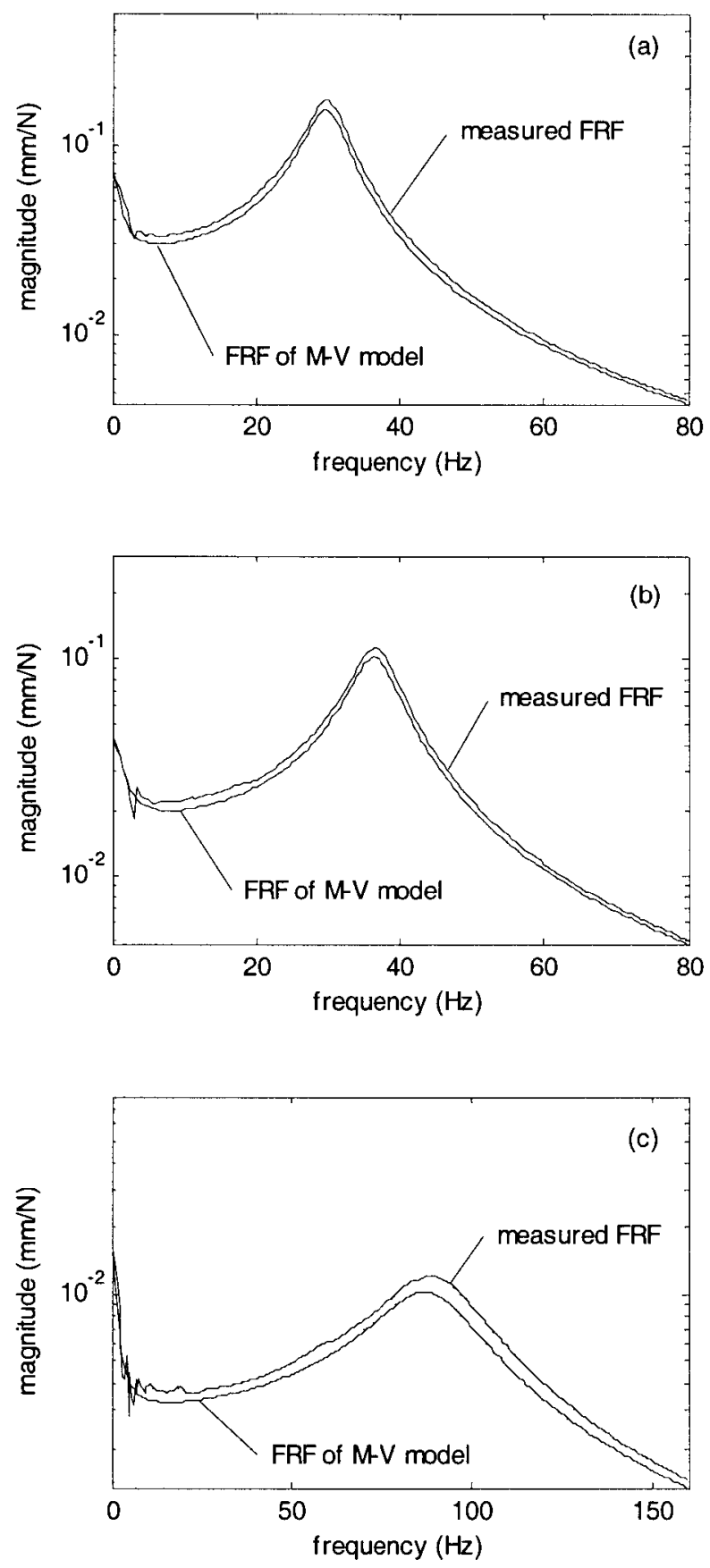

Fig. 5.7. Frequency response spectra of (a) bubble mount; (b) plate mount; (c) rubber stud (Trial 1). 

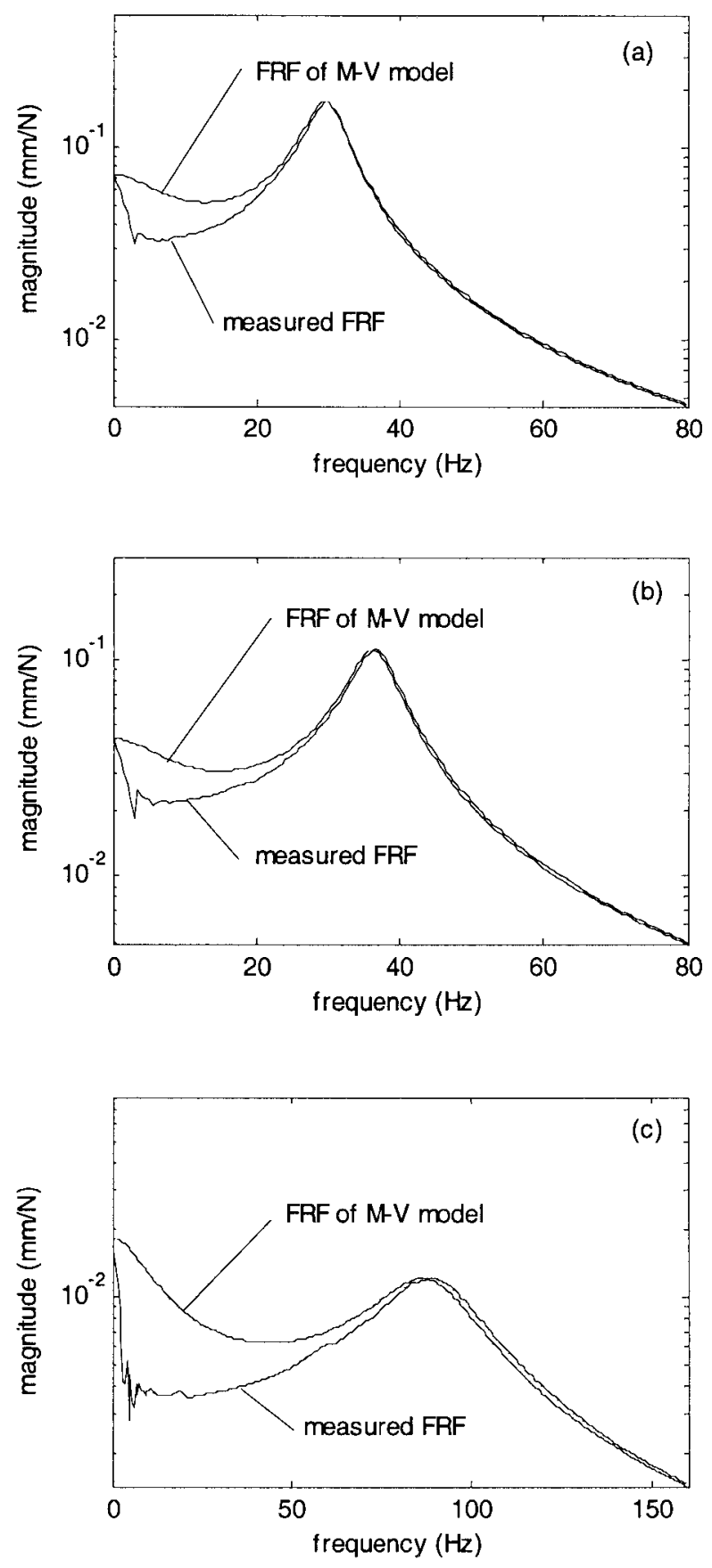

Fig. 5.8. Frequency response spectra of (a) bubble mount; (b) plate mount; (c) rubber stud (Trial 2). 


\subsubsection{Parameter identification of $M-M-V$ models by constrain optimization}

The results of section 5.3.2 illustrate that the M-V models do not match measured frequency response curve very well over the entire frequency range. From Chapter 3 , the case that M-V model can not simulate well the system which consists of Type B and Type $C$ Maxwell elements. So, the method developed in chapter 4 is used to identify the parameters of rubber isolator models with two Maxwell elements (M-M-V models). The resulting parameters are listed in Table 5.6. If the resulting Maxwell elements of the models belong to Type $B$ and Type $C$, the conclusion in Chapter 4 will be proved; i.e., the two Maxwell elements in identified M-M-V model which can accurately simulate the static and dynamic behavior of Maxwell system should belong to Type $B$ and Type $C$ respectively.

Since the values of $\tau_{1}$ and $\tau_{2}$ in Table 5.6 are very different for all three models, it is easy to conclude that the two Maxwell elements in each model belong to a different Type. Also, note the negative values identified for the primary damper $c$. Consequently, since the model's overall damping must be positive, there must be at least one Maxwell element in each of the M-M-V models that belong to Type $A$ or $B$ since only Type $A$ and $B$ elements contribute damping to the system (Type $C$ elements are stiffness dominant and therefore do not add damping). Because the M-V models in Table 5.4 present correct natural frequencies and damping ratios and the identified Maxwell elements belong to Type $C$ which is stiffness dominant, the primary damper $c$ present all the damping effect of the mounting system. The combined damping effect of the Type $A$ or $B$ element and 
the primary damper $c$ should be equivalent to the effect of the primary damper $c$ of $M-V$ Models in Table 5.4. If the Maxwell element with $\tau_{1}$ is damping dominant (Type A), the sum of $c$ and $c_{1}$ in Table 5.6 should be similar to the value of $c$ in Table 5.4. By comparing the data in Table 5.4 and 5.6, it is found that the sum of $c$ and $c_{1}$ in Table 5.6 are much larger than to the value of $c$ in Table 5.4 for all three models. Therefore, it is concluded that the Maxwell element with $\tau_{1}$ in each model is not Type A but Type B. Since $\tau_{2}$ is much large than $\tau_{1}$, the second Maxwell element should belong to Type $C$ for all three models. This result matches the conclusion in Chapter 4 where the two Maxwell elements identified in the M-M-V model belong to Type $B$ and Type $C$.

Since Type B elements also contribute stiffness, the combined stiffness effect of the Type $B$ and $C$ elements should be equivalent to the effect of Type $C$ elements of $\mathrm{M}-\mathrm{V}$ models in Table 5.4. Since the stiffness values $k_{2}$ of the stiffness dominant elements in Table 5.6 are obviously smaller than the stiffness values $k_{1}$ in Table 5.4, the Maxwell element with $\tau_{1}$ should belong to Type $B$ or $C$ in order to contribute stiffness effect to the systems. Also, the sum of $k_{1}$ and $k_{2}$ in Table 5.6 are obviously larger than the stiffness values $k_{1}$ in Table 5.4. Therefore, at least one of the Maxwell elements of M-M-V models is not Type C. This again demonstrates that the two identified Maxwell elements of the M-M-V models in Table 5.6 belong to Type B and Type $C$.

The frequency response of identified M-M-V models and experimental system are show in Fig. 5.9. As shown, the frequency response of models match much closer to the experimentally measured frequency response compared with the results from section 5.3.2. 
Table 5.6. Parameters of identified general Maxwell models.

\begin{tabular}{|c|c|c|c|}
\hline Part number & Bubble mount & Plate mount & Rubber stud \\
\hline$m(\mathrm{~kg})$ & 1.04 & 1.04 & 1.04 \\
\hline$k(\mathrm{kN} / \mathrm{m})$ & 14.01 & 23.15 & 54.51 \\
\hline$c(\mathrm{~N}-\mathrm{s} / \mathrm{m})$ & -55.49 & -29.15 & -70.18 \\
\hline$k_{1}(\mathrm{kN} / \mathrm{m})$ & 64.44 & 45.21 & 389.70 \\
\hline$c_{1}(\mathrm{~N}-\mathrm{s} / \mathrm{m})$ & 87.81 & 70.93 & 239.46 \\
\hline$\tau_{1}(\mathrm{~ms})[$ Type $]$ & $1.36[B]$ & $1.57[B]$ & $0.61[B]$ \\
\hline$k_{2}(\mathrm{kN} / \mathrm{m})$ & 18.23 & 26.05 & 224.11 \\
\hline$c_{2}(\mathrm{~N}-\mathrm{s} / \mathrm{m})$ & 2297.39 & 2402.82 & 15507.4 \\
\hline$\tau_{2}(\mathrm{~ms})[$ Type $]$ & $126.01[C]$ & $92.24[C]$ & $69.20[C]$ \\
\hline
\end{tabular}



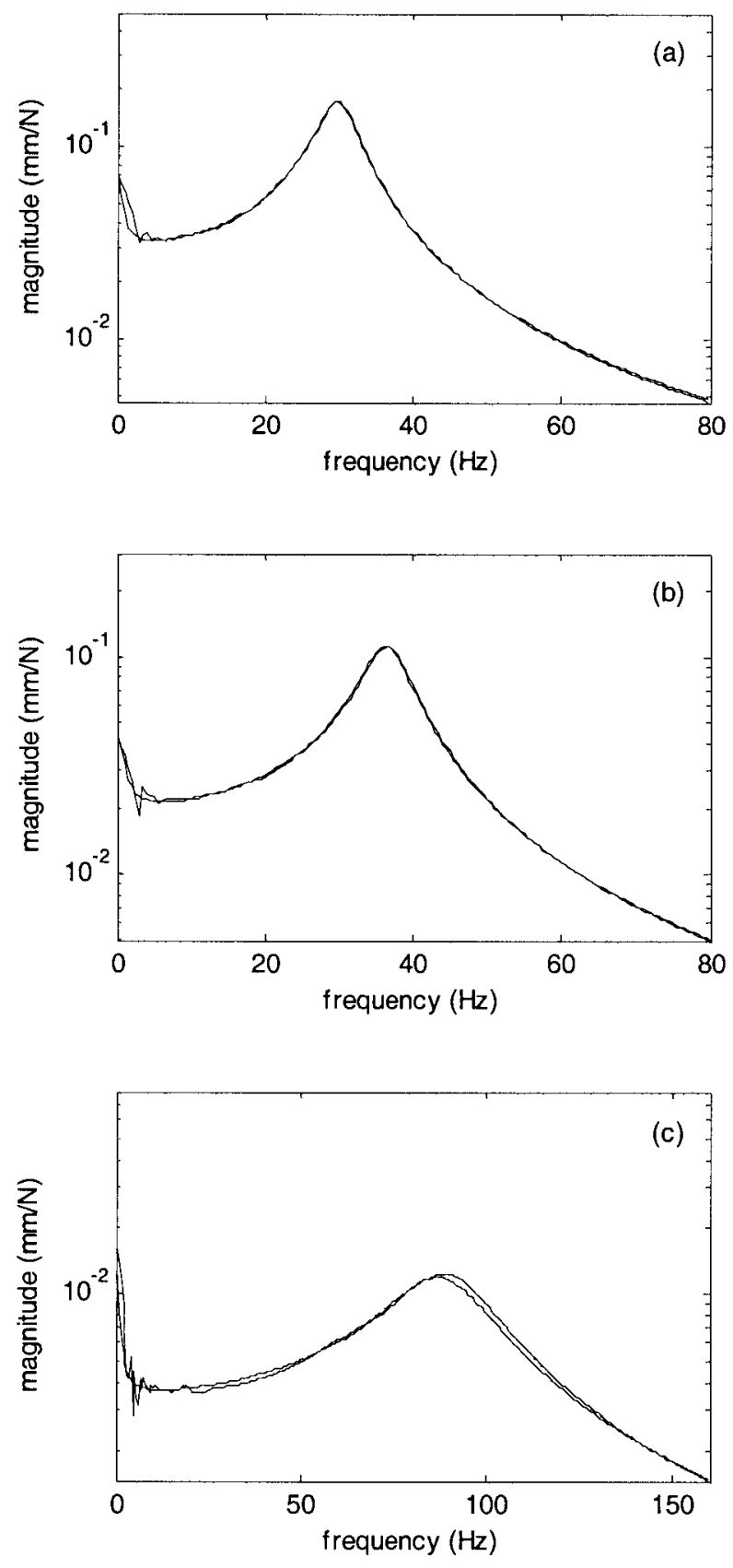

Fig. 5.9. Frequency response spectra of (a) bubble mount; (b) plate mount; (c) rubber stud. 


\subsection{Summary}

Experiments on real elastomeric isolators are conducted with three different rubber isolators subjected to both static and dynamic experiments. For all three rubber isolators, it is shown that although identified stiffness consistent Voigt models can match the static stiffness, natural frequency and damping ratio, they cannot match the measured frequency response. Meanwhile, identified mass consistent Voigt models match measured natural frequencies and damping ratios, although they cannot match the measured static stiffness and frequency response. Identified Maxwell models having only one Maxwell element can match the measured natural frequency, damping ratio and static stiffness, but cannot match the measured frequency response curves well. However, using the method developed in this article, identified Maxwell models having two Maxwell elements can accurately represent the measured static and dynamic characteristics of real elastomeric isolation systems. 


\section{CHAPTER 6}

\section{DYNAMIC ANALYSIS OF AN AEROELASTIC AIRCRAFT WING WITH DYNAMIC VIBRATION ABSORBER}

\subsection{Dynamic Analysis of an Aeroelastic Aircraft Wing}

\subsubsection{Problem formulation}

The aeroelastic airfoil is a flexible structure residing in a steady-state airflow. The dynamic properties of this system such as natural frequencies and damping ratios change as flight parameters (such as airspeed $U$ ) and external condition (such as air density $\rho$ ) change. A two-degree-of-freedom analytical model of a pitch-plunge airfoil is illustrated in Fig. 6.1.

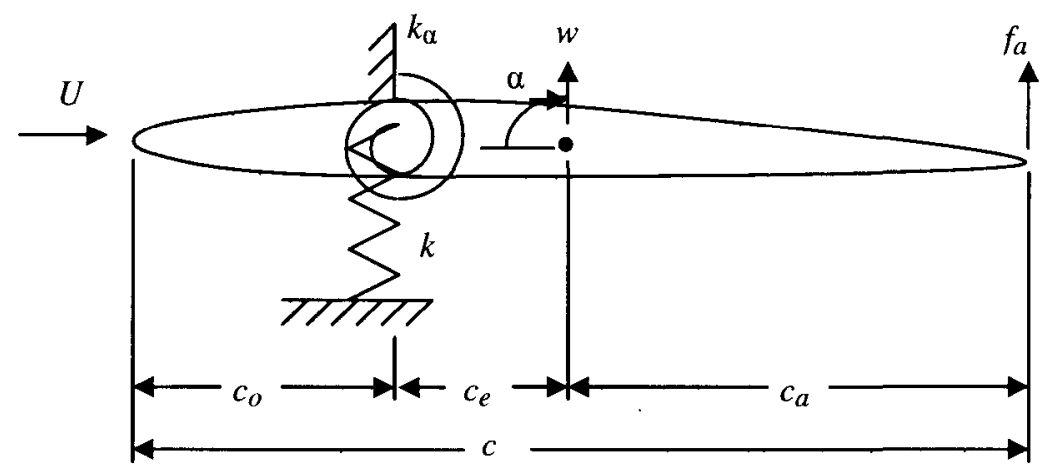

Fig. 6.1. Analytical model of pitch-plunge airfoil.

Consider the pitch-plunge airfoil having a straight elastic axis perpendicular to the fuselage which is assumed to be fixed in space. The deformation can be measured by 
deflection $w$ and rotation $\alpha$. The governing equations of motion of the analytical model are [13],

$$
\begin{aligned}
& m \ddot{w}=-k w-\beta k \dot{w}+k c_{e} \alpha+\beta k c_{e} \dot{\alpha}-F_{w}+f_{a} \\
& I \ddot{\alpha}=k c_{e} w+\beta k c_{e} \dot{w}-\left(k_{\alpha}+k c_{e}^{2}\right) \alpha-\beta\left(k_{\alpha}+k c_{e}^{2}\right) \dot{\alpha}-T_{\alpha}-c_{a} f_{a}
\end{aligned}
$$

from reference [47],

$$
\begin{aligned}
& F_{w}=U^{2} \frac{\rho c}{2} \frac{d C_{L}}{d \alpha} \alpha+U \frac{\rho c}{2} \frac{d C_{L}}{d \alpha} \dot{w}+U \frac{\rho c}{2} \frac{d C_{L}}{d \alpha}\left(\frac{3 c}{4}-c_{0}\right) \dot{\alpha} \\
& T_{\alpha}=U^{2} \frac{\rho c}{2} \frac{d C_{L}}{d \alpha}\left(\frac{c}{4}-c_{0}\right) \alpha+U \frac{\rho c}{2} \frac{d C_{L}}{d \alpha}\left(\frac{c}{4}-c_{0}\right) \dot{w}+U \frac{\rho c}{2} \frac{d C_{L}}{d \alpha}\left[\left(\frac{c}{4}-c_{0}\right)\left(\frac{3 c}{4}-c_{0}\right)+\frac{\pi}{8 \frac{d C_{L}}{d \alpha}}\right] \dot{\alpha}
\end{aligned}
$$

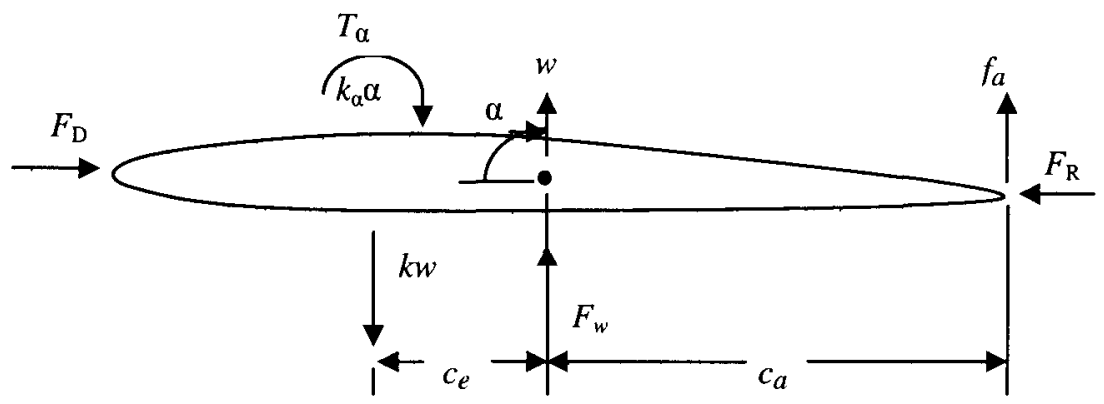

Fig. 6.2. Dynamic analysis of pitch-plunge airfoil.

Then, the governing equations of motion

$$
\mathbf{M} \ddot{\mathbf{x}}+(\mathbf{C}+U \mathbf{L}) \dot{\mathbf{x}}+\left(\mathbf{K}+U^{2} \mathbf{H}\right) \mathbf{x}=\mathbf{b} f_{a}
$$

where

$$
\begin{aligned}
& \mathbf{x}=\left[\begin{array}{l}
w \\
\alpha
\end{array}\right], \quad \mathbf{b}=\left[\begin{array}{c}
1 \\
-c_{a}
\end{array}\right], \quad \mathbf{M}=\left[\begin{array}{ll}
m & 0 \\
0 & I
\end{array}\right], \quad \mathbf{C}=\beta \mathbf{K}, \quad \mathbf{K}=\left[\begin{array}{cc}
k & -k c_{e} \\
-k c_{e} & k_{\alpha}+k c_{e}^{2}
\end{array}\right], \\
& \mathbf{H}=\frac{\rho c}{2} \frac{d C_{L}}{d \alpha}\left[\begin{array}{cc}
0 & 1 \\
0 & c\left(\frac{1}{4}-\frac{c_{o}}{c}\right)
\end{array}\right], \quad \mathbf{L}=\frac{\rho c}{2} \frac{d C_{L}}{d \alpha}\left[\begin{array}{cc}
1 & c\left(\frac{3}{4}-\frac{c_{o}}{c}\right) \\
c\left(\frac{1}{4}-\frac{c_{o}}{c}\right) & c^{2}\left[\left(\frac{1}{4}-\frac{c_{o}}{c}\right)\left(\frac{3}{4}-\frac{c_{o}}{c}\right)+\frac{\pi}{8 \frac{d C_{L}}{d \alpha}}\right]
\end{array}\right]
\end{aligned}
$$


In Eq. (2), the translational and rotational structural stiffnesses of the wing are described by $k$ and $k_{\alpha}$ respectively, $m$ and $I$ are the mass and mass moment of inertia about the center of gravity of the wing, and $\beta$ is the proportionality constant for this proportionally damped system. The derivative $d C_{L} / d \alpha$ is assumed to be constant, $U$ is the airspeed and $\rho$ is the density of air. The external force $f_{a}$ is from aileron excitation. $L$ and $H$ are from the aeroelasticity theory of cantilever wing [47].

\subsubsection{Modal analysis}

Rewrite Eq. (6.3) in state-space form,

$$
\dot{\mathbf{y}}=\mathbf{A y}+\mathbf{Q}
$$

where

$$
\begin{aligned}
& \mathbf{A}=\left[\begin{array}{cccc}
0 & 0 & 1 & 0 \\
0 & 0 & 0 & 1 \\
-\frac{k}{m} & \frac{k c_{e}}{m}-U^{2} \frac{\rho c}{2 m} \frac{d C_{L}}{d \alpha} & \frac{-\beta k}{m}-U \frac{\rho c}{2 m} \frac{d C_{L}}{d \alpha} & \frac{\beta k c_{e}}{m}-U \frac{\rho c^{2}}{2 m} \frac{d C_{L}}{d \alpha}\left(\frac{3}{4}-\frac{c_{0}}{c}\right) \\
\frac{k c_{e}}{I}-\frac{k_{\alpha}+k c_{e}^{2}}{I}-U^{2} \frac{\rho c^{2}}{2 I} \frac{d C_{L}}{d \alpha}\left(\frac{1}{4}-\frac{c_{0}}{c}\right) & \frac{\beta k c_{e}}{I}-U \frac{\rho c^{2}}{2 I} \frac{d C_{L}}{d \alpha}\left(\frac{1}{4}-\frac{c_{0}}{c}\right) & -\frac{\beta\left(k_{\alpha}+k c_{e}^{2}\right)}{I}-U \frac{\rho c^{3}}{2 I}\left[\frac{d C_{L}}{d \alpha}\left(\frac{3}{16}-\frac{c_{0}}{c}+\frac{c_{0}^{2}}{c^{2}}\right)+\frac{\pi}{8}\right]
\end{array}\right] \\
& \mathbf{y}=\left[\begin{array}{c}
w \\
\alpha \\
\dot{w} \\
\dot{\alpha}
\end{array}\right], \quad \mathbf{Q}=\left[\begin{array}{c}
0 \\
0 \\
\frac{f_{u}}{m} \\
-\frac{c_{a} f_{a}}{I}
\end{array}\right]
\end{aligned}
$$

The modal analysis is based on the eigenvalue problem of system matrix $\mathbf{A}$ in Eq. (6.6). Due to the presence of airspeed $U$ in system matrix $\mathbf{A}$, the natural frequencies and damping ratios change as airspeed changes. The natural frequencies and damping ratios versus airspeed are shown in Fig. 6.2. When the airspeed exceeds the critical airspeed $U_{\mathrm{cr}}$, the damping ratio of totational model is negative, and the system is unstable. 

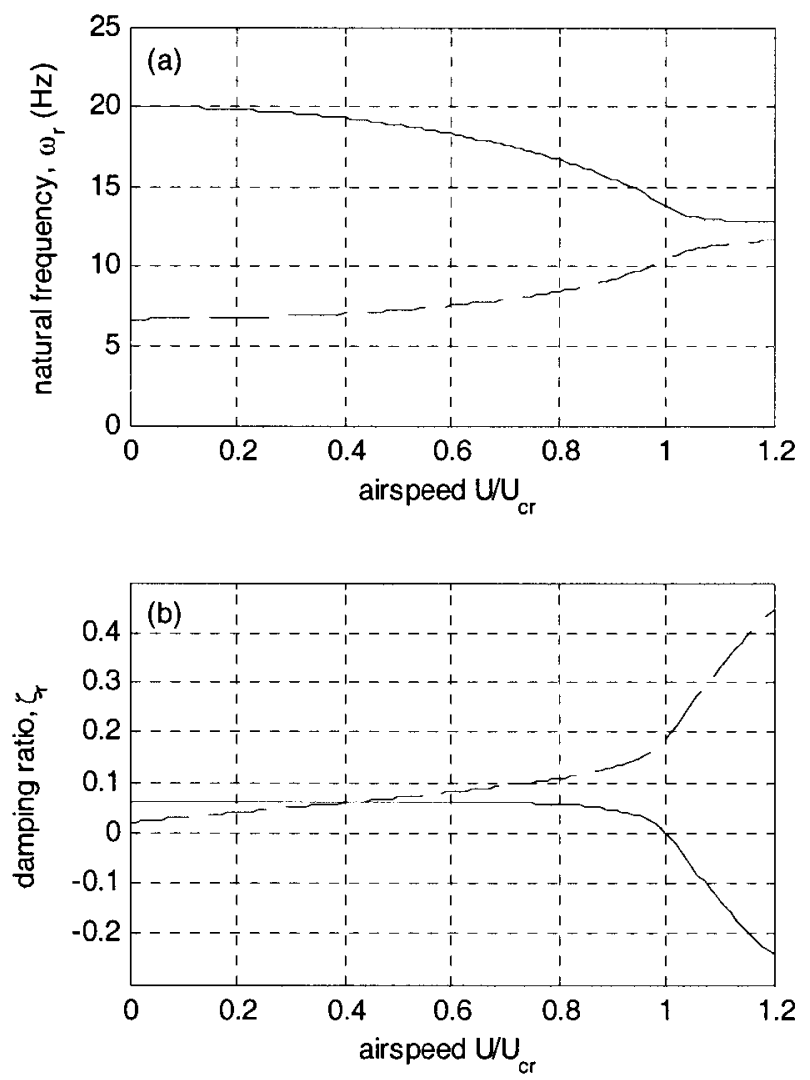

Fig. 6.2. Natural frequencies and damping ratios versus airspeed velocity. "___ “ rotational model; " - -" translational model.

\subsubsection{Impulse response analysis}

From $\mathrm{Eq}(6.5)$, the time response of system can be expressed using the transition matrix method [43],

$$
y(k+1)=\Phi y(k)+\Gamma Q(k)
$$

where, 


$$
\Phi=e^{A T}=I+T A+\frac{T^{2}}{2 !} A^{2}+\frac{T^{3}}{3 !} A^{3}+\cdots
$$

and

$$
\Gamma=A^{-1}(\Phi-I)
$$

In Eq. (6.8), $T$ is time increment of calculation. In the case of the external disturbance of $1 \mathrm{kN}$ impulse force at aileron, the impulse responses of pitch-plunge airfoil at different airspeed are calculated and shown in Fig. 6.3. The unit of vertical coordinate is rotation in one thousandth of radian and the unit of horizontal coordinate is time in second. In the cases of the airspeed is less than $U_{\mathrm{cr}}$, the system is stable and the impulse time responses vanish quickly. In the case of the airspeed equal to $U_{\mathrm{cr}}$ the vibration lasts for a long time before it vanishes. In the cases of the airspeed is higher than $U_{\mathrm{cr}}$, the system is unstable and the vibration will continuously increase. These results are consistent with the results of modal analysis. 

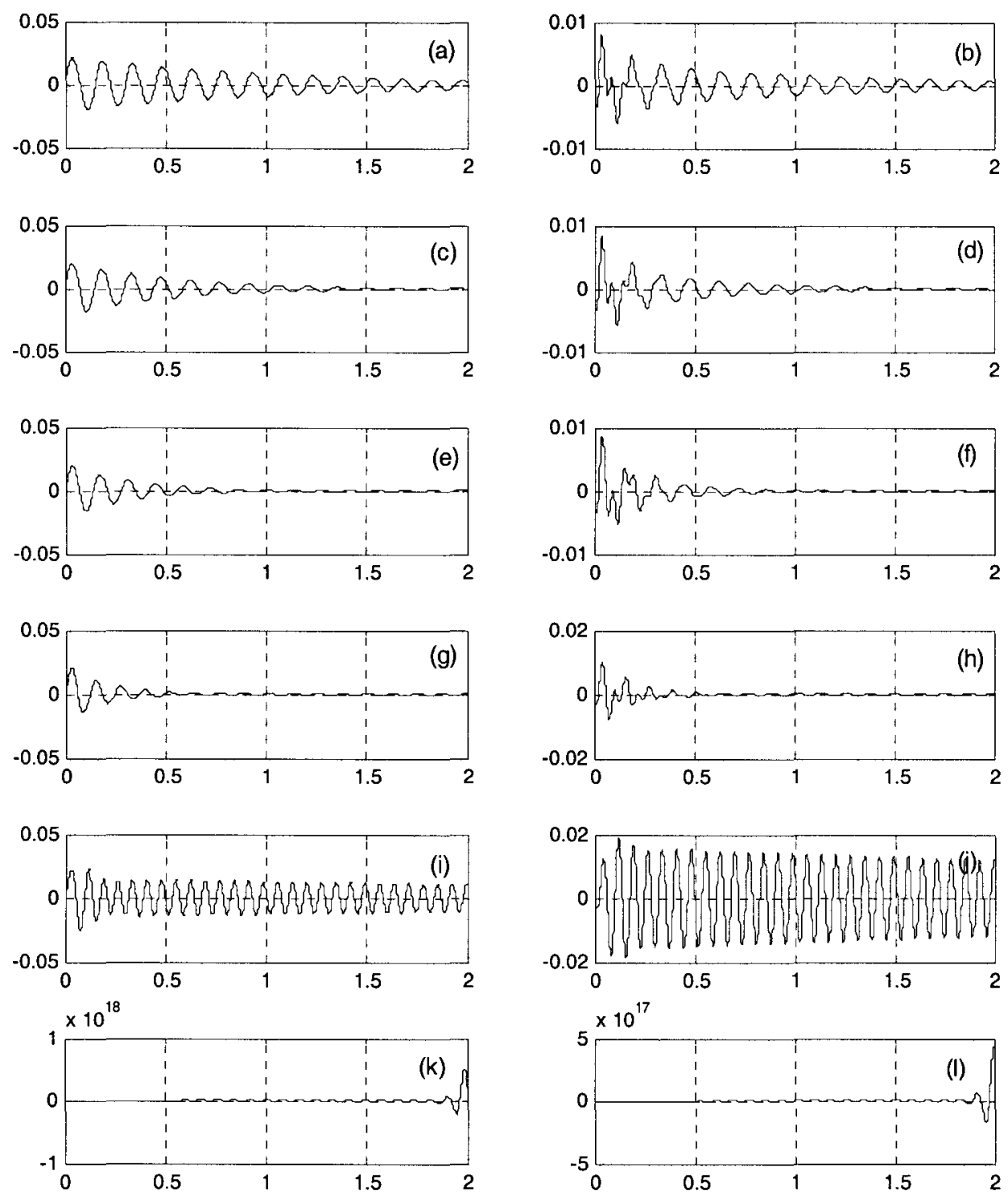

Fig. 6.3. Impulse time responses of pitch-plunge airfoil. (a, b) $U=0$; (c, d) $U=0.25 U_{\text {cr; }}$; (e, f) $U=0.5 U_{\mathrm{cr}} ;(\mathrm{g}, \mathrm{h}) U=0.75 U_{\mathrm{cr}} ;(\mathrm{i}, \mathrm{j}) U=U_{\mathrm{cr}} ;(\mathrm{k}, \mathrm{l}) U=1.25 U_{\mathrm{cr}} ;(\mathrm{a}, \mathrm{c}, \mathrm{e}, \mathrm{g}, \mathrm{i}, \mathrm{k})$ translational movement $w ;(\mathrm{b}, \mathrm{d}, \mathrm{f}, \mathrm{h}, \mathrm{j}, \mathrm{l})$ rotational movement $\alpha$. 
The time response of the pitch-plunge airfoil under random external excitation is simulated with airspeed accelerating continuously from $U / U_{\mathrm{cr}}=0.8$ to 1.2 at a linear rate for 30 seconds. The external excitation $f_{\mathrm{a}}$ with mean zero and standard deviation $1 \mathrm{kN}$ is applied over the 30 second time period. Results are shown in Fig. 6.4 where the translational displacement $w$ and the rotational movement $\alpha$ are stable at the cases of the airspeeds under $U_{\mathrm{cr}}$, but increase very quickly after the airspeed reaches $U_{\mathrm{cr}}$.
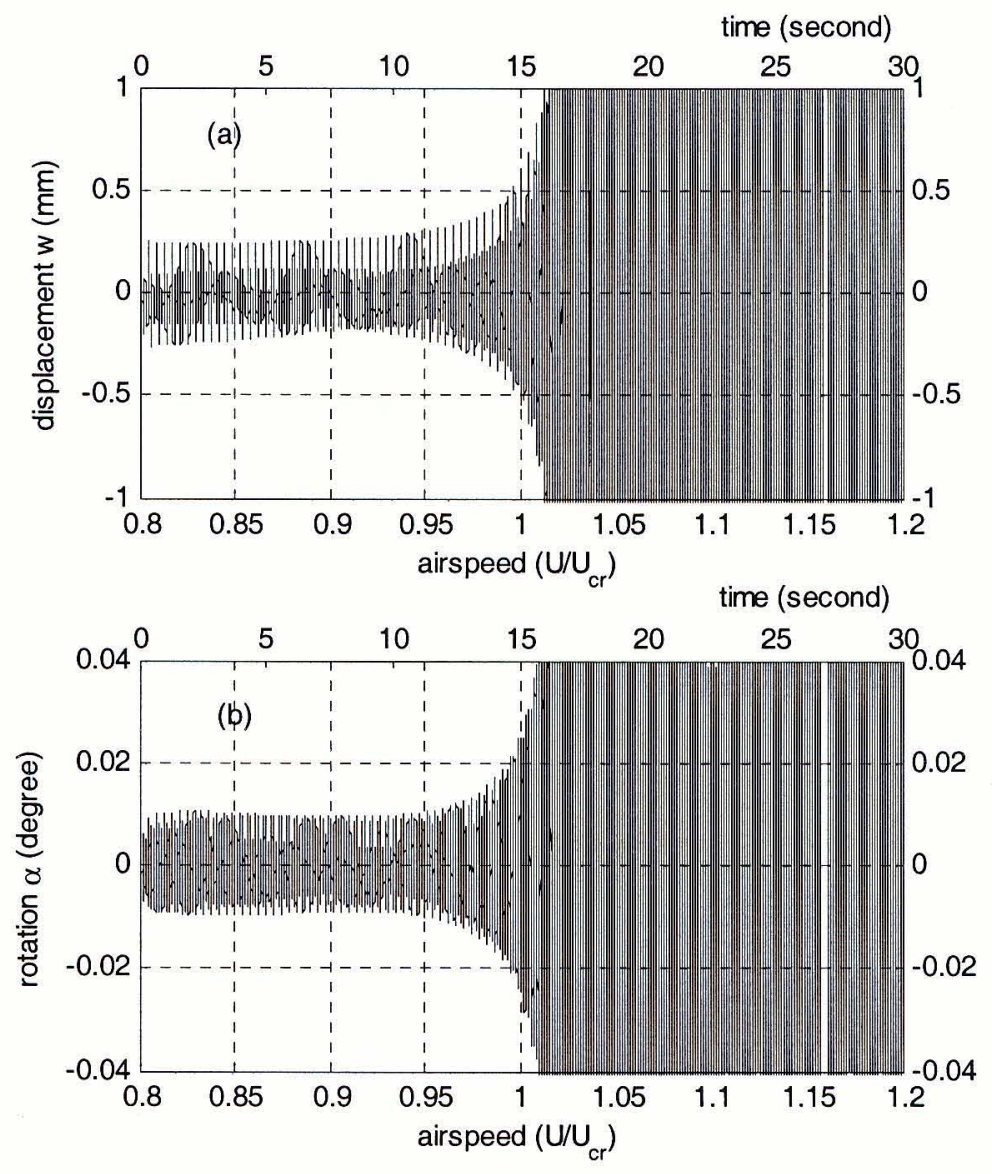

Fig. 6.4. Flight simulation of aeroelastic aircraft wing. 


\subsection{Dynamic Analysis of an Aeroelastic Aircraft Wing with DVA}

\subsubsection{Problem formulation}

In order to increases the critical airspeed and improve the dynamic properties of the pitch-plunge airfoil at higher airspeed, the application of DVA (dynamic vibration absorber) is investigated for the aircraft wing vibration suppression. The three-degree-offreedom analytical model of pitch-plunge airfoil with a DVA is illustrated in Fig. 6.5.

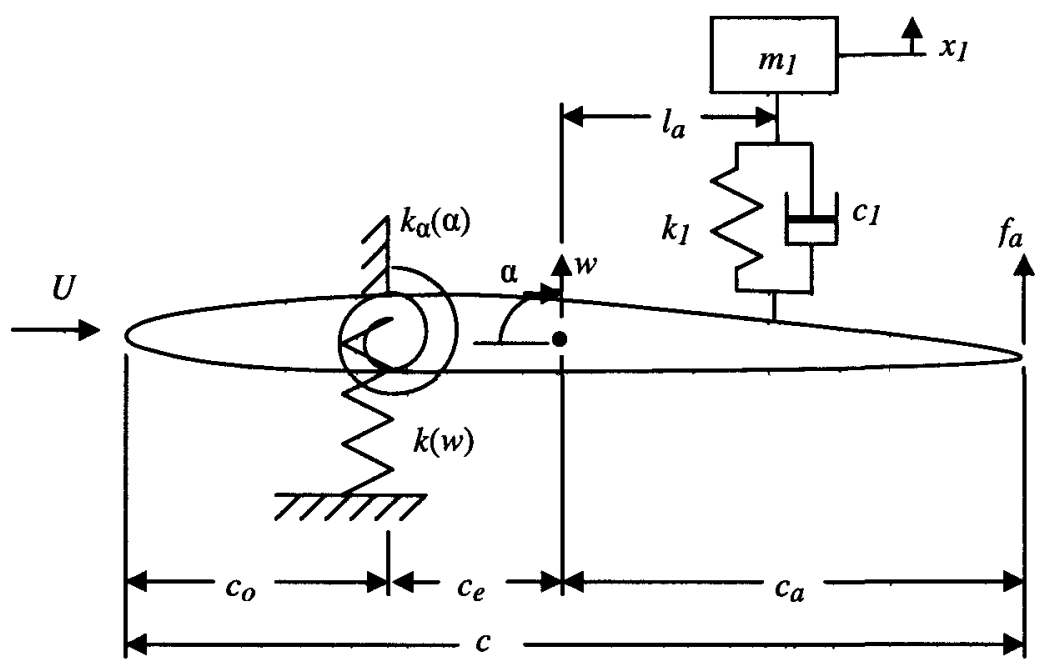

Fig. 6.5. Analytical model of pitch-plunge airfoil with absorber.

The governing equations of motion of the analytical model are,

$$
\mathbf{M} \ddot{\mathbf{x}}+(\mathbf{C}+U \mathbf{L}+\mathbf{D}) \dot{\mathbf{x}}+\left(\mathbf{K}+U^{2} \mathbf{H}+\mathbf{G}\right) \mathbf{x}=\mathbf{b} f_{a}
$$

where 


$$
\begin{aligned}
& \mathbf{x}=\left[\begin{array}{c}
w \\
\alpha \\
x_{1}
\end{array}\right], \quad \mathbf{b}=\left[\begin{array}{c}
1 \\
-c_{a} \\
0
\end{array}\right], \quad \mathbf{M}=\left[\begin{array}{ccc}
m & 0 & 0 \\
0 & I & 0 \\
0 & 0 & m_{1}
\end{array}\right], \quad \mathbf{C}=\boldsymbol{\beta} \mathbf{K}, \\
& \mathbf{K}=\left[\begin{array}{ccc}
k & -k c_{e} & 0 \\
-k c_{e} & k_{\alpha}+k c_{e}^{2} & 0 \\
0 & 0 & 0
\end{array}\right], \quad \mathbf{H}=\frac{\rho c}{2} \frac{d C_{L}}{d \alpha}\left[\begin{array}{ccc}
0 & 1 & 0 \\
0 & c\left(\frac{1}{4}-\frac{c_{0}}{c}\right) & 0 \\
0 & 0 & 0
\end{array}\right] \text {, } \\
& \mathbf{L}=\frac{\rho c}{2} \frac{d C_{L}}{d \alpha}\left[\begin{array}{ccc}
1 & c\left(\frac{3}{4}-\frac{c_{0}}{c}\right) & 0 \\
c\left(\frac{1}{4}-\frac{c_{0}}{c}\right) & c^{2}\left[\left(\frac{1}{4}-\frac{c_{0}}{c}\right)\left(\frac{3}{4}-\frac{c_{0}}{c}\right)+\frac{\pi}{8 \frac{d C_{L}}{d \alpha}}\right. & 0 \\
0 & 0 & 0
\end{array}\right] \\
& \mathbf{D}=c_{1}\left[\begin{array}{ccc}
1 & -l_{a} & -1 \\
-l_{a} & l_{a}^{2} & l_{a} \\
-1 & l_{a} & 1
\end{array}\right], \quad \mathbf{G}=k_{1}\left[\begin{array}{ccc}
1 & -l_{a} & -1 \\
-l_{a} & l_{a}^{2} & l_{a} \\
-1 & l_{a} & 1
\end{array}\right]
\end{aligned}
$$

The parameters of the DVA, mass $m_{1}$, stiffness $k_{1}$, damping $c_{1}$ and distance from DVA to the gravity center of the wing $l_{\alpha}$, will be determined in the following section.

Equation (6.10) can also be written in state-space form as Eq. (6.5), but the matrix $\mathbf{A}$ and vectors $\mathbf{y}$ and $\mathbf{Q}$ are different from Eq. (6.6). For this analytical model,

$$
\begin{aligned}
& \mathbf{A}=\left[\begin{array}{cccccc}
0 & 0 & 0 & 1 & 0 & 0 \\
0 & 0 & 0 & 0 & 1 & 0 \\
0 & 0 & 0 & 0 & 0 & 1 \\
-\frac{K_{11}+U^{2} H_{11}+G_{11}}{m} & -\frac{K_{12}+U^{2} H_{12}+G_{12}}{m} & -\frac{K_{13}+U^{2} H_{13}+G_{13}}{m} & -\frac{C_{11}+U L_{11}+D_{11}}{m} & -\frac{C_{12}+U L_{12}+D_{12}}{m} & -\frac{C_{13}+U L_{13}+D_{13}}{m} \\
-\frac{K_{21}+U^{2} H_{21}+G_{21}}{I} & -\frac{K_{22}+U^{2} H_{22}+G_{22}}{I} & -\frac{K_{23}+U^{2} H_{23}+G_{23}}{I} & -\frac{C_{21}+U L_{21}+D_{21}}{I} & -\frac{C_{22}+U L_{22}+D_{22}}{I}-\frac{C_{23}+U L_{23}+D_{23}}{I} \\
-\frac{K_{31}+U^{2} H_{31}+G_{31}}{m_{1}} & -\frac{K_{32}+U^{2} H_{32}+G_{32}}{m_{1}} & -\frac{K_{33}+U^{2} H_{33}+G_{33}}{m_{1}} & -\frac{C_{31}+U L_{31}+D_{31}}{m_{1}} & -\frac{C_{32}+U L_{32}+D_{32}}{m_{1}}-\frac{C_{33}+U L_{33}+D_{33}}{m_{1}}
\end{array}\right] \\
& \mathbf{y}=\left[\begin{array}{c}
w \\
\alpha \\
x_{1} \\
\dot{w} \\
\dot{\alpha} \\
\dot{x}_{1}
\end{array}\right], \quad \mathbf{Q}=\left[\begin{array}{c}
0 \\
0 \\
0 \\
\frac{f_{a}}{m} \\
-\frac{c_{a} f_{a}}{I} \\
0
\end{array}\right]
\end{aligned}
$$


The modal analysis is based on the eigenvalue problem of system matrix $\mathbf{A}$ in Eq. (6.12). The time response analysis is based on the same equation as in Eq. (6.7-9). The matrix $\mathbf{A}$ and vector $\mathbf{y}$ and $\mathbf{Q}$ are from Eq. (6.12).

\subsubsection{Optimized parameters of DVA}

The parameters of DVA need to be decided to maximize the minimum damping ratio and get the desired property. The limitation is from the constraints of aircraft design. For this example, let $m_{1} / m=0.04$ and the DVA is put near the front tip. The minimum damping ratios of system with DVA which has variable parameters of $c_{1}$ and $k_{1}$ are shown in Fig. 6.6-6.9. They are the results of system simulation with the airspeed $U_{\mathrm{r}}$ $=0.5 U_{\mathrm{cr}}\left(\right.$ Fig 5(a, b, c)), $U_{\text {cr }}$ (Fig. 5(d, e, f)), $1.06 U_{\text {cr }}$ (Fig. 5(g, h, i)) and $1.12 U_{\text {cr }}$ (Fig. 5(j, $\mathrm{k}, 1))$ separately.

From these figures, two basic conclusions can be drawn: First, the damping ratio of system change as the values of $k_{1}$ and $c_{1}$ change. For all the different airspeed conditions which are illustrated in Fig. 6.6, every surface of minimum damping ratio has only one maximum point. That means the optimized parameters of $k_{1}$ and $c_{1}$ exists at the maximum point. The optimization method can be applied for searching the optimized value of $k_{1}$ and $c_{1}$. Second, at low airspeed, such as $U=0.5 U_{\mathrm{cr}}$, the minimum damping ratio with optimized values of $k_{1}$ and $c_{1}$ changes slightly in a small range as the values of $k_{1}$ and $c_{1}$ change. The system is stable in a wide range of values of $k_{1}$ and $c_{1}$. As the airspeed increases to $U=U_{\text {cr }}$, this is the critical airspeed of the system without the application of DVA, the system is stable with the application of DVA. The influence of the values of $k_{1}$ and $c_{1}$ on minimum damping ratio increases. The minimum damping 
ratio can be higher than the minimum damping ratio in the case of airspeed $U=0.5 U_{\mathrm{cr}}$ if the values of $k_{1}$ and $c_{1}$ are around the optimized position. Out of this optimized range, the minimum damping ratio is lower than the minimum damping ratio values in the case of airspeed $U=0.5 U_{\mathrm{cr}}$. As the airspeed keep increases, such as $U=1.06 U_{\mathrm{cr}}$, the system can be stable only in a specific range of values of $k_{1}$ and $c_{1}$. Out of this range the system will be unstable. As the airspeed increases to $U=1.12 U_{\mathrm{cr}}$, the system is stable only in a very small range. The influence of parameter of $k_{1}$ and $c_{1}$ on stability of system increases dramatically. 

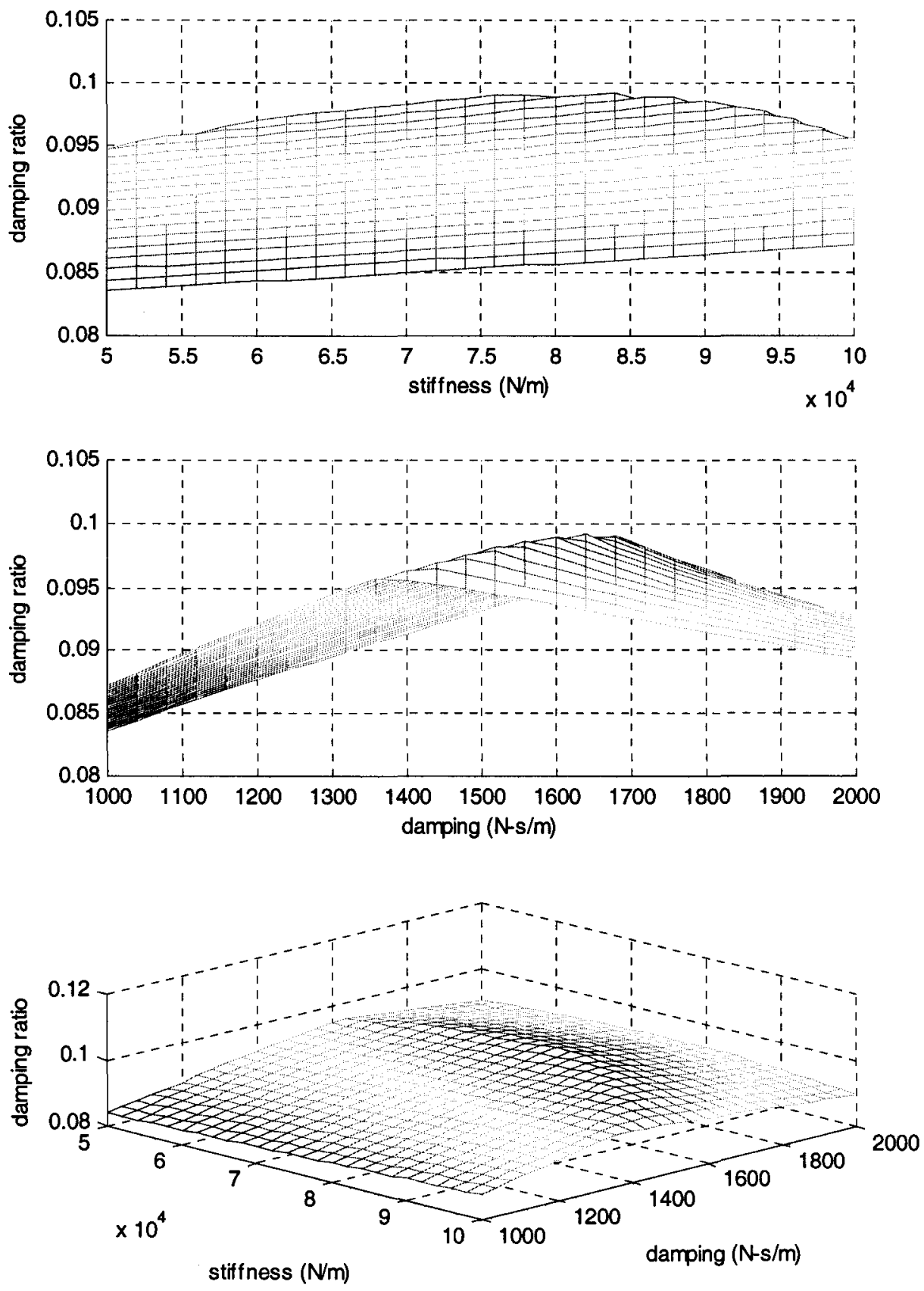

Fig. 6.6. Minimum damping ratios versus DVA parameters when airspeed $U=0.5 U_{\mathrm{cr}}$. 

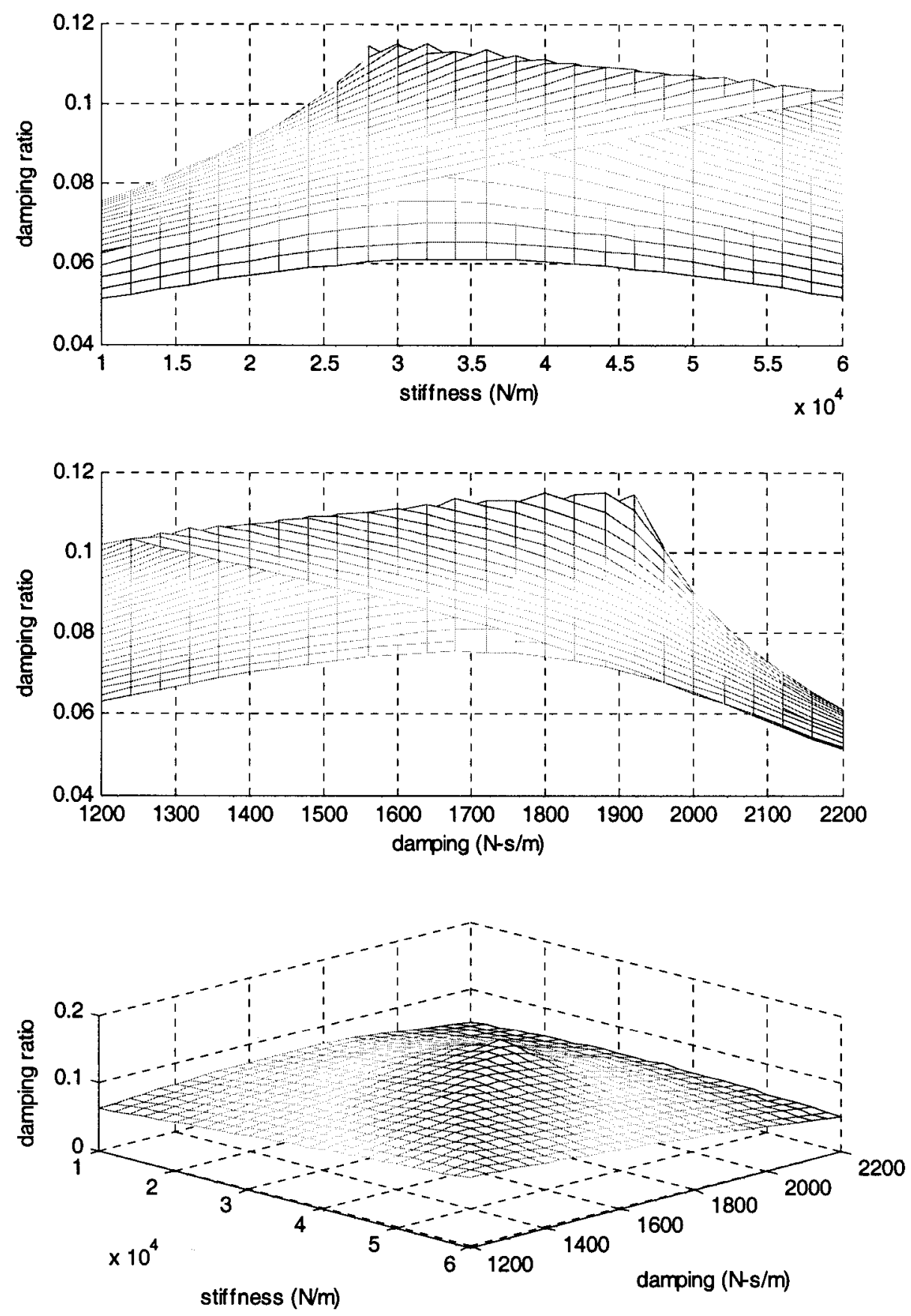

Fig. 6.7. Minimum damping ratios versus DVA parameters when airspeed $U=U_{\mathrm{cr}}$. 

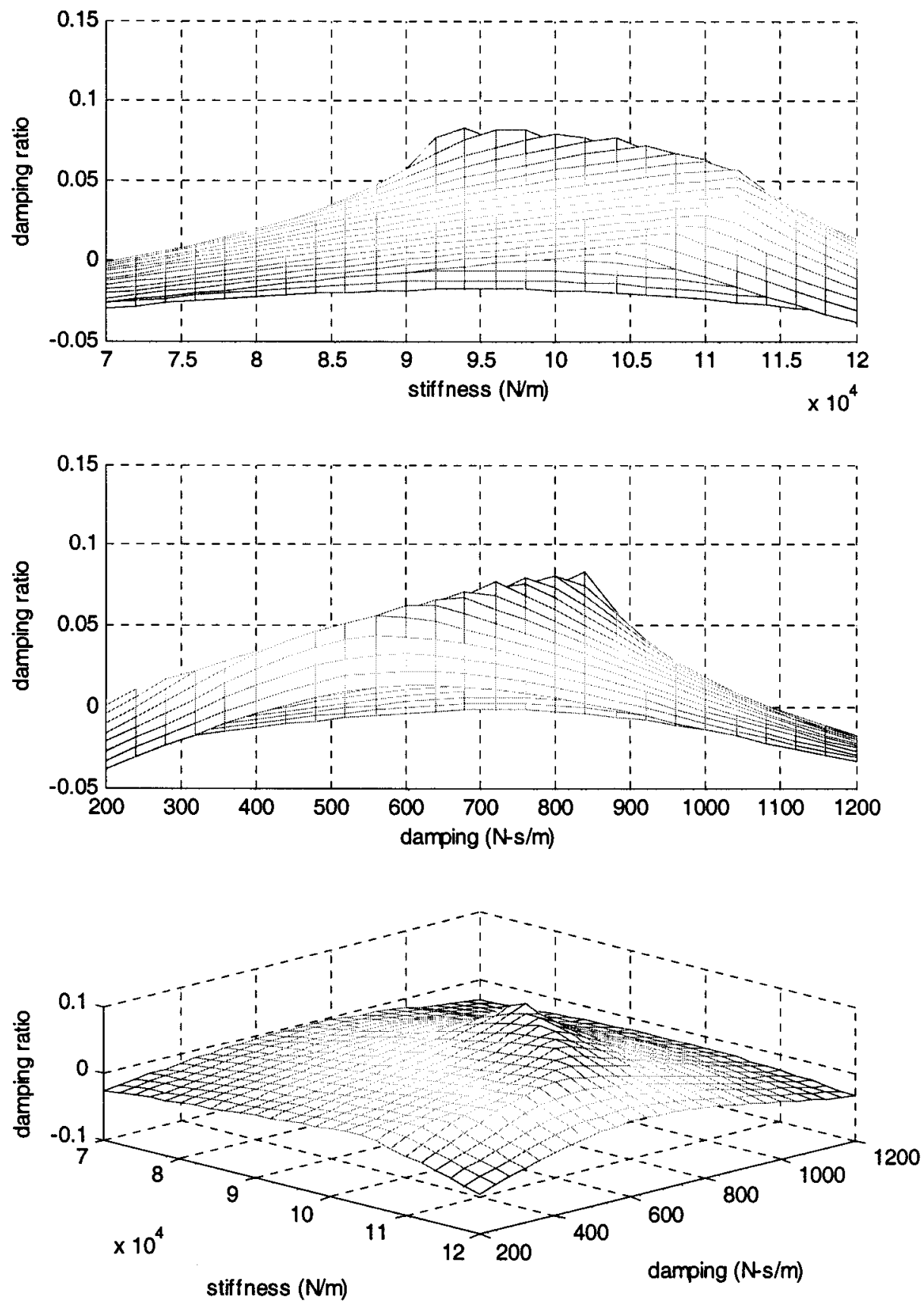

Fig. 6.8. Minimum damping ratios versus DVA parameters when airspeed $U=1.06 U_{\mathrm{cr}}$. 

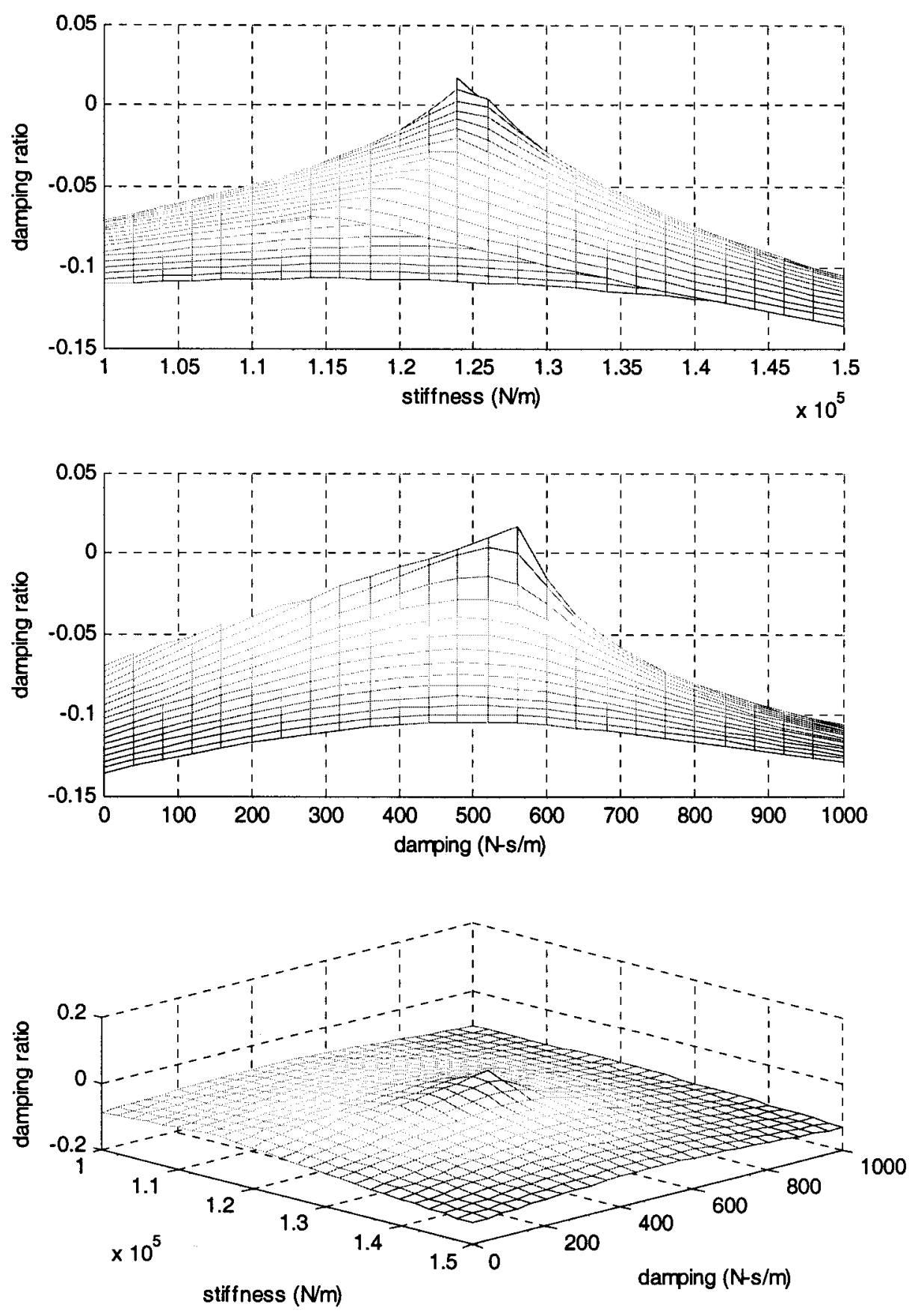

Fig. 6.9. Minimum damping ratios versus DVA parameters when airspeed $U=1.12 U_{\mathrm{cr}}$. 
The distance from DVA to the gravity center of the wing $l_{\mathrm{a}}$, influences the properties of vibration suppression. Figure 6.10 shows the minimum damping ratio as airspeed changes with different value of $l_{\mathrm{a}}$ and optimized values of $c_{1}$ and $k_{1}$. The mass $m_{1} / m=0.04$. Because the purpose of DVA is to suppress the vibration at high airspeed, the judgment for the position of DVA is based on the critical airspeed where the system turns to be unstable. From Fig. 6.10, the critical airspeeds for different distances from DVA to the gravity center of the wing are listed in Table 6.1. The DVA performs better as the value of $l_{\mathrm{a}}$ increases for this specific example. The limitation is from structure design of the wing. From Table 6.1, the best result is from the case the DVA is placed at the front tip.

Table 6.1. Critical airspeed with different DVA position.

\begin{tabular}{|c|c|}
\hline Position & Critical airspeed $\left(U / U_{\mathrm{cr}}\right)$ \\
\hline Rear tip & 1.07 \\
\hline Rear middle & 1.06 \\
\hline Front middle & 1.09 \\
\hline Front tip & 1.14 \\
\hline
\end{tabular}



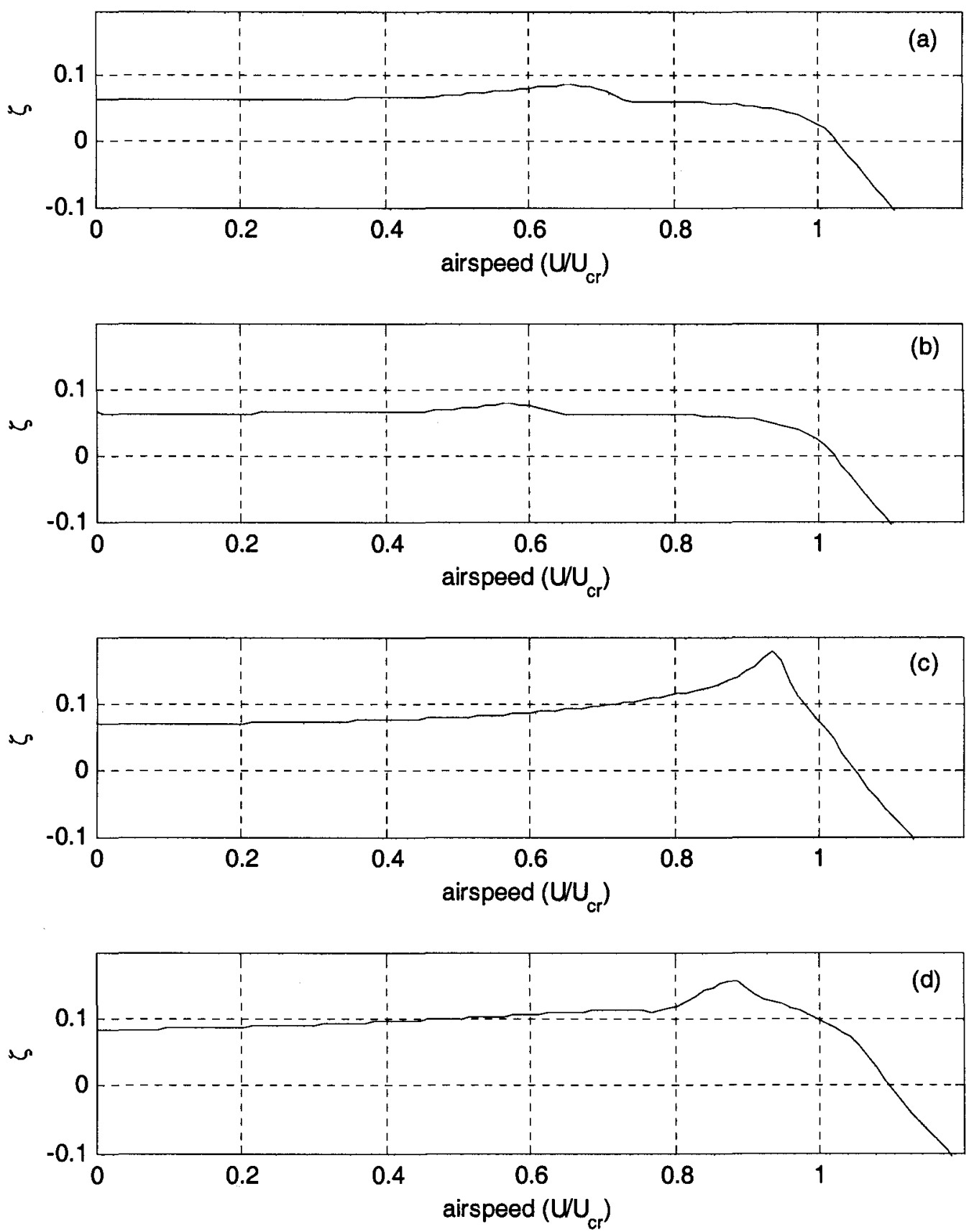

Fig. 6.10. Optimized Minimum damping ratios where the position of DVA is (a) rear tip;

(b) rear middle; (c) front middle; (d) front tip. 
The mass of DVA is another factor which influences the effects of vibration suppression. Figure 6.11 shows the optimized minimum damping ratio as airspeed changes with different DVA mass and optimized $c_{1}$ and $k_{1}$. The position of the DVA is close to the front tip. From Fig. 6.11, the critical airspeeds for different mass are listed in Table 6.2. The DVA performs better as the mass increases. The limitation is from the constraints of aircraft design. From Table 6.2, the best result is from the case where $m_{1} / m$ $=0.04$.

Table 6.2. Critical airspeed with different DVA mass.

\begin{tabular}{|c|c|}
\hline$m_{1} / m$ & Critical airspeed $\left(U / U_{\mathrm{cr}}\right)$ \\
\hline 0.01 & 1.08 \\
\hline 0.02 & 1.10 \\
\hline 0.03 & 1.12 \\
\hline 0.04 & 1.14 \\
\hline
\end{tabular}



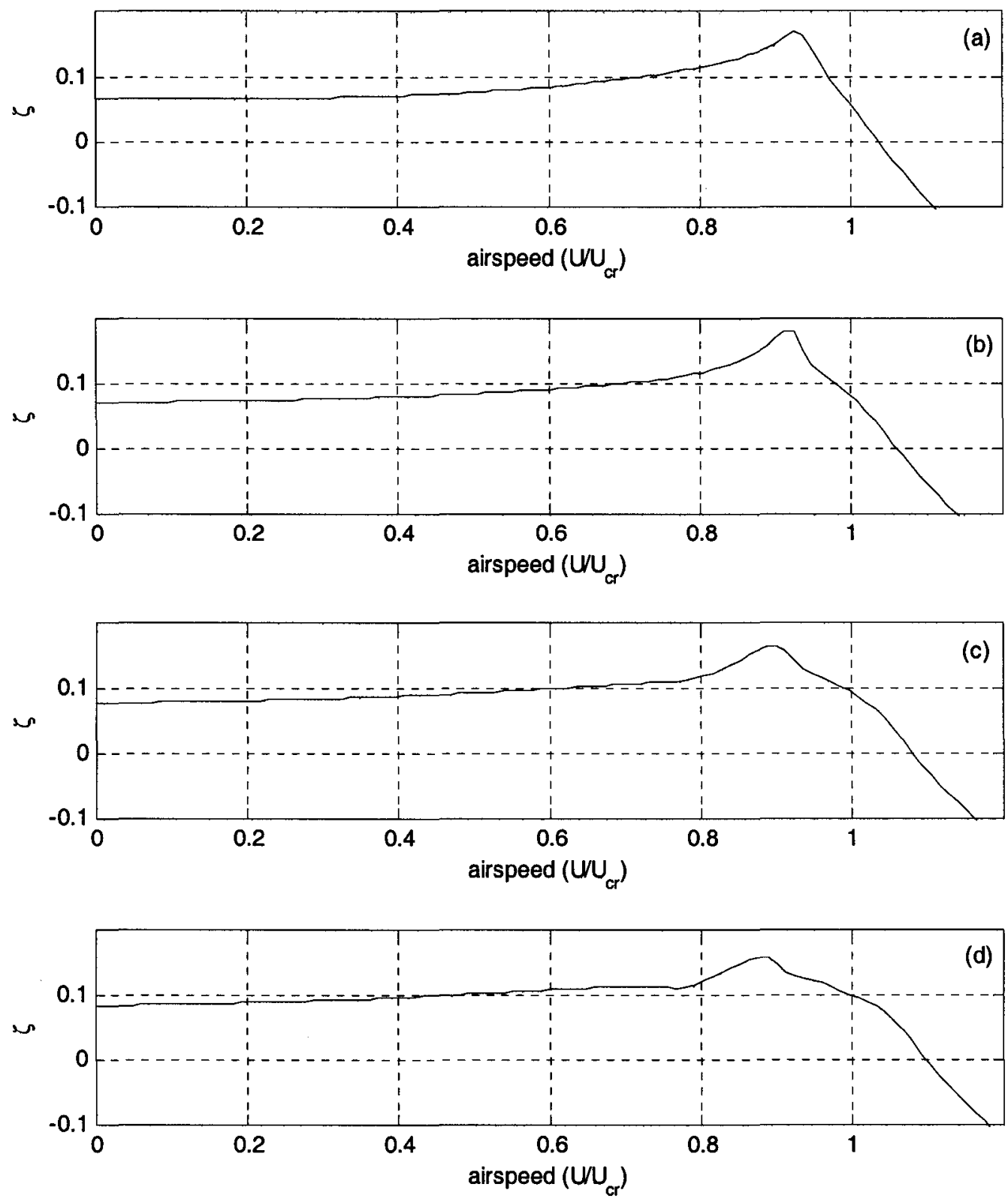

Fig. 6.11. Optimized Minimum damping ratios where mass is (a) 0.01 ; (b) 0.02 ; (c) 0.03 ; (d) 0.04 . 
In the case of the DVA is placed at the front tip and the $m_{1} / m=0.04$, the optimized DVA parameters, $k_{1}$ and $c_{1}$, are shown in Fig. 6.12. The associated minimum damping ratios are already shown in Fig. 6.10(d) and Fig. 6.11(d).
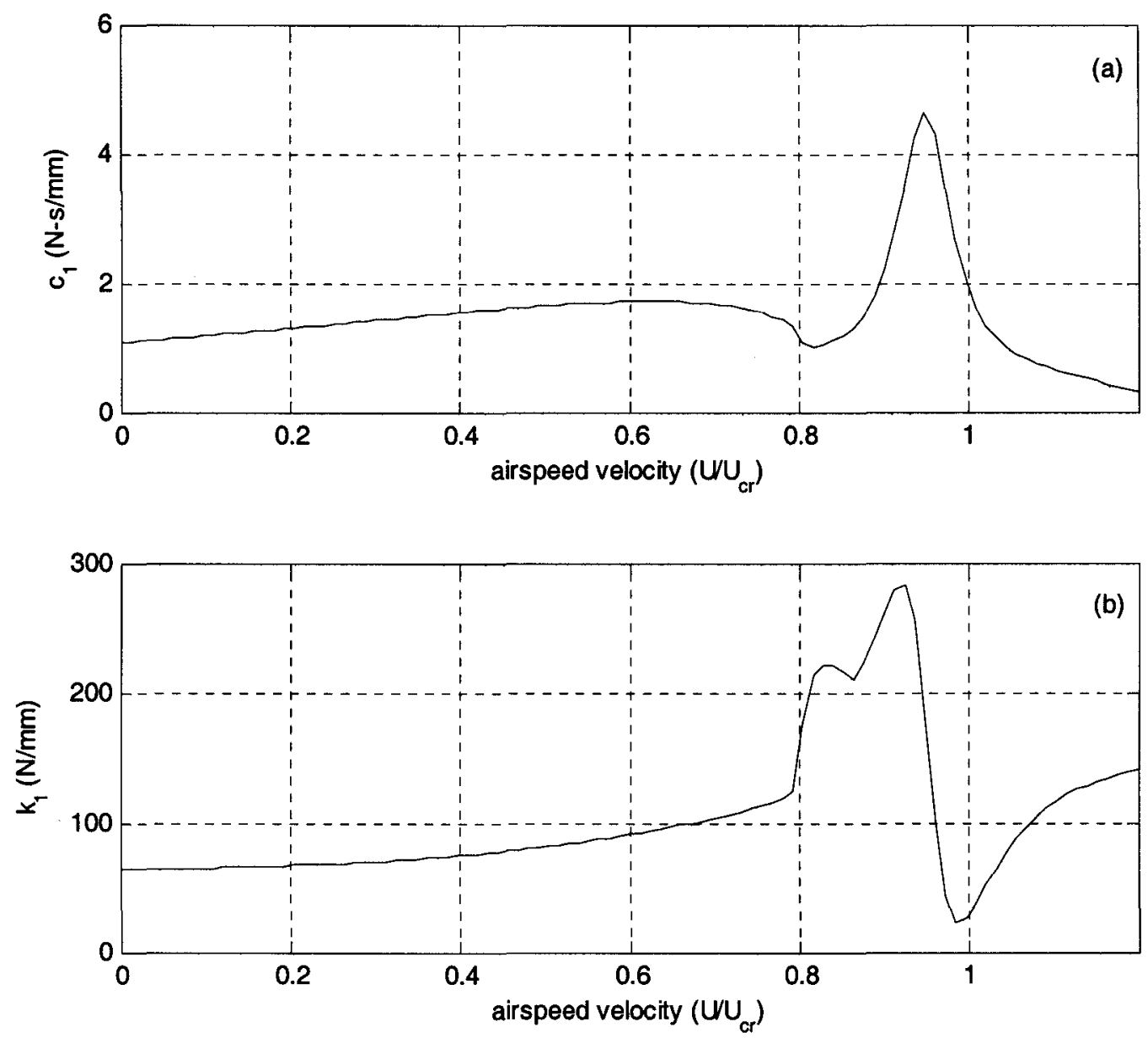

Fig. 6.12. Optimized values of DVA parameters $c_{1}$ and $k_{1}$. 


\subsubsection{Comparison with the performance of constant parameter DVA}

For the tunable DVA control, both the values of $k_{1}$ and $c_{1}$ need to be controlled based on the values shown in Fig. 6.12. Unfortunately, the device for tunable stiffness is not well developed yet. Tuning both $k_{1}$ and $c_{1}$ at the same time according the values shown in Fig. 6.12 is very difficult. The constant parameter DVA is an alternative way due to its simplicity. But the effect of vibration suppression of constant parameter DVA needs to be investigated and compared with the effects of tunable DVA. From Fig. 6.12, three DVA are designed based on the parameters values at the airspeed $U / U_{\mathrm{cr}}=1,1.06$ and 1.12. The parameters of three DVAs are listed in Table 6.3. The minimum damping ratios are shown in Fig. 6.13. It is found that the constant DVA(2) which is designed based on the airspeed $U / U_{\mathrm{cr}}=1.06$ have best vibration suppression effect. Its critical airspeed $U / U_{\mathrm{cr}}=1.074$. It is very different from the vibration suppression effect of tunable DVA whose critical airspeed $U / U_{\mathrm{cr}}=1.141$.

Table 6.3. Critical airspeed with different constant DVA.

\begin{tabular}{|c|c|c|c|}
\hline Model & $k_{1}(\mathrm{~N} / \mathrm{mm})$ & $c_{1}(\mathrm{~N}-\mathrm{s} / \mathrm{mm})$ & Critical airspeed ratio $\left(U_{\text {cr }}\right)$ \\
\hline Constant DVA(1) & 29.38 & 1.88 & 1.02 \\
\hline Constant DVA(2) & 91.56 & 0.86 & 1.07 \\
\hline Constant DVA(3) & 123.16 & 0.59 & 1.04 \\
\hline
\end{tabular}



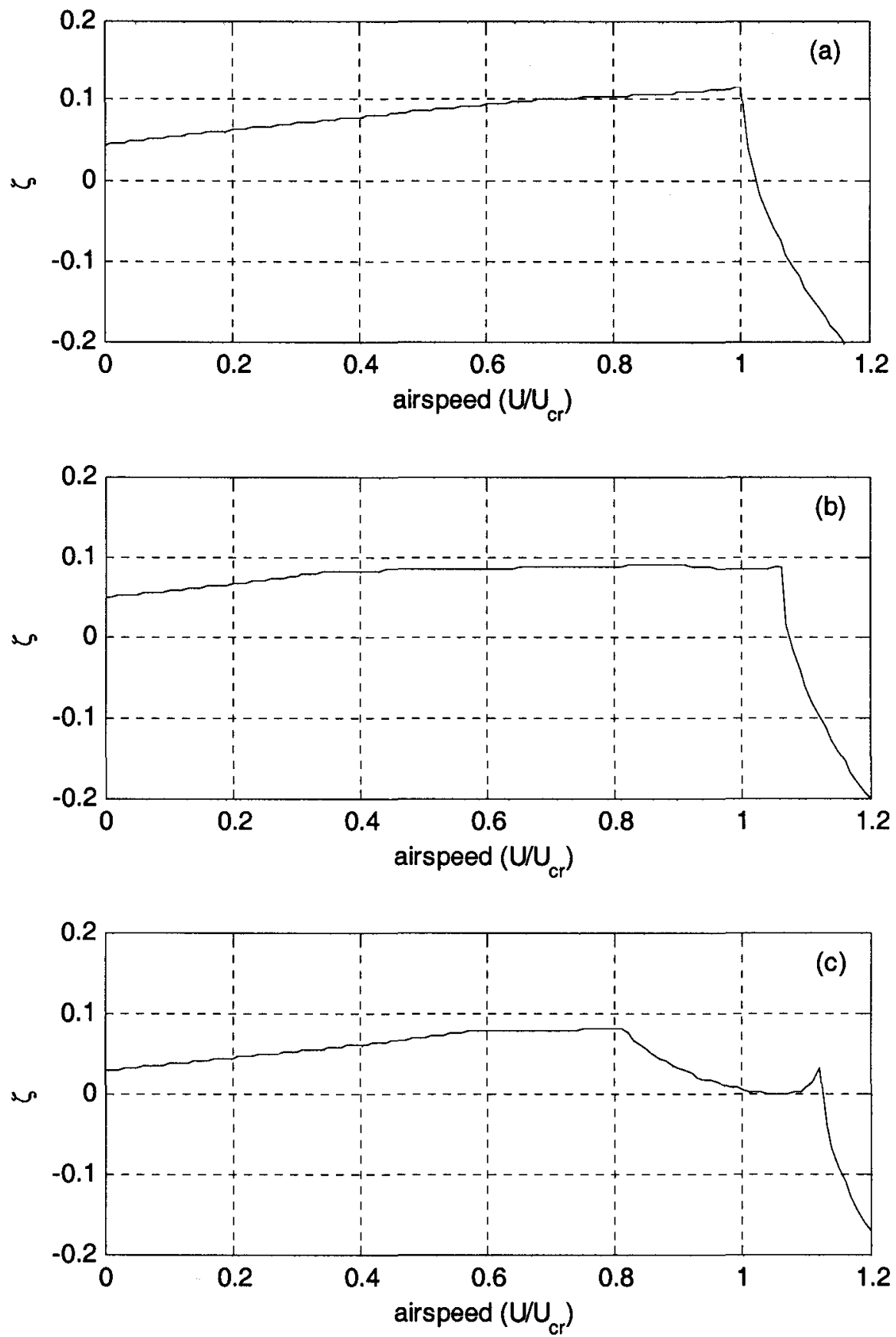

Fig. 6.13. Minimum damping ratios of constant DVA vs. airspeed. 


\subsubsection{Simulation results}

The time response of the pitch-plunge airfoil under random external excitation is simulated with airspeed accelerating continuously from $U / U_{\mathrm{cr}}=0.8$ to 1.2 at a linear rate for 30 seconds. The external excitation $f_{\mathrm{a}}$ with mean zero and standard deviation $1 \mathrm{kN}$ is applied over the 30 second time period. Figure 6.14-6.16 shows the time responses of the pitch-plunge airfoil with DVA(1), DVA(2), DVA(3). The time responses of the pitchplunge airfoil with tunable DVA whose parameters are controlled based on the data are shown in Fig. 6.12. The translational displacement $w$ and the rotational movement $\alpha$ increase very quickly after the airspeed reaches $U=1.19 U_{\mathrm{cr}}$. The behavior of tunable DVA is much better than the behavior of any constant parameter DVA. 

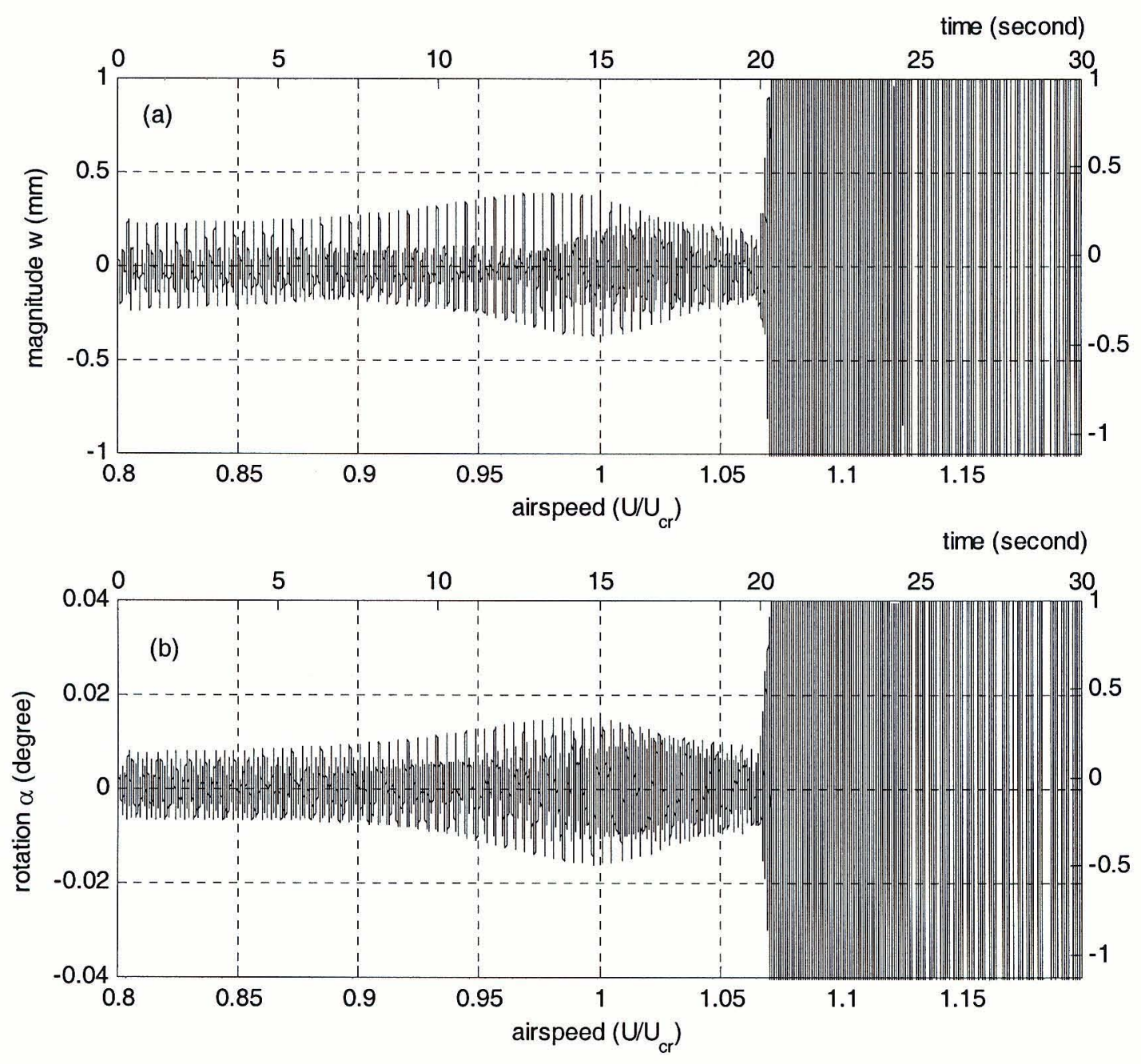

Fig. 6.14. Time responses of pitch-plunge airfoil with constant DVA(1) versus airspeed. 

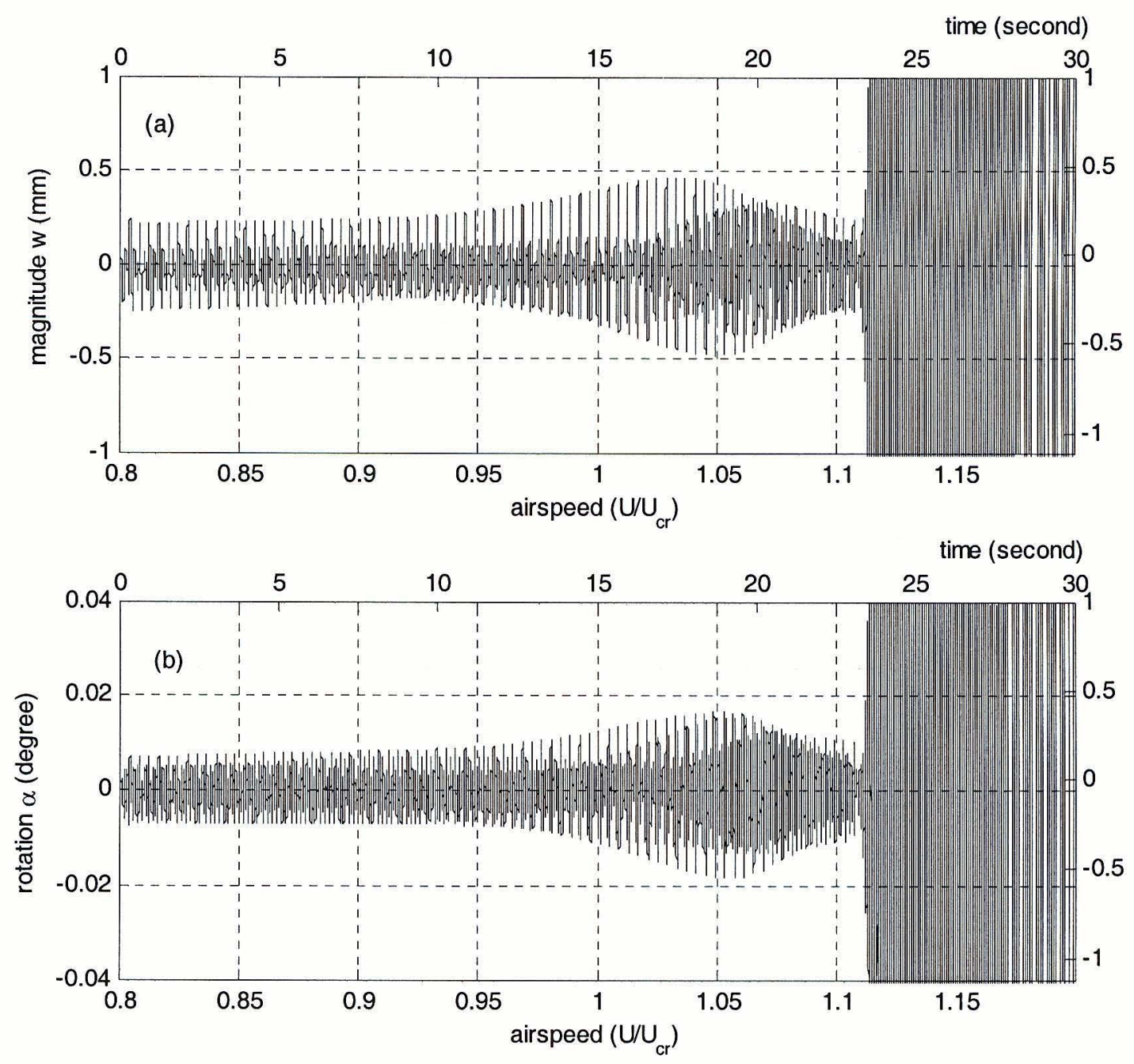

Fig. 6.15. Time responses of pitch-plunge airfoil with constant DVA(2) versus airspeed. 

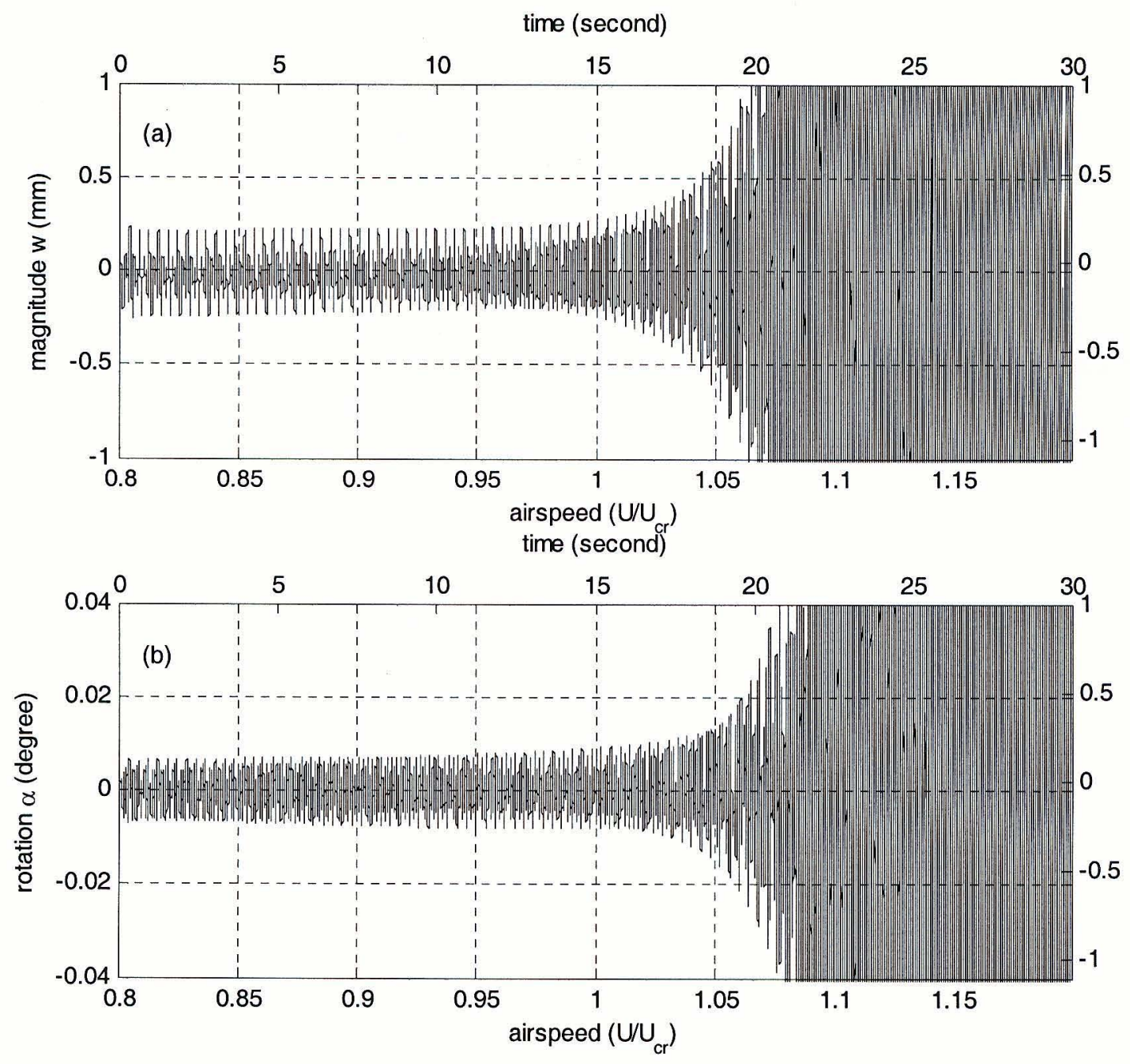

Fig. 6.16. Time responses of pitch-plunge airfoil with constant DVA(3) versus airspeed. 


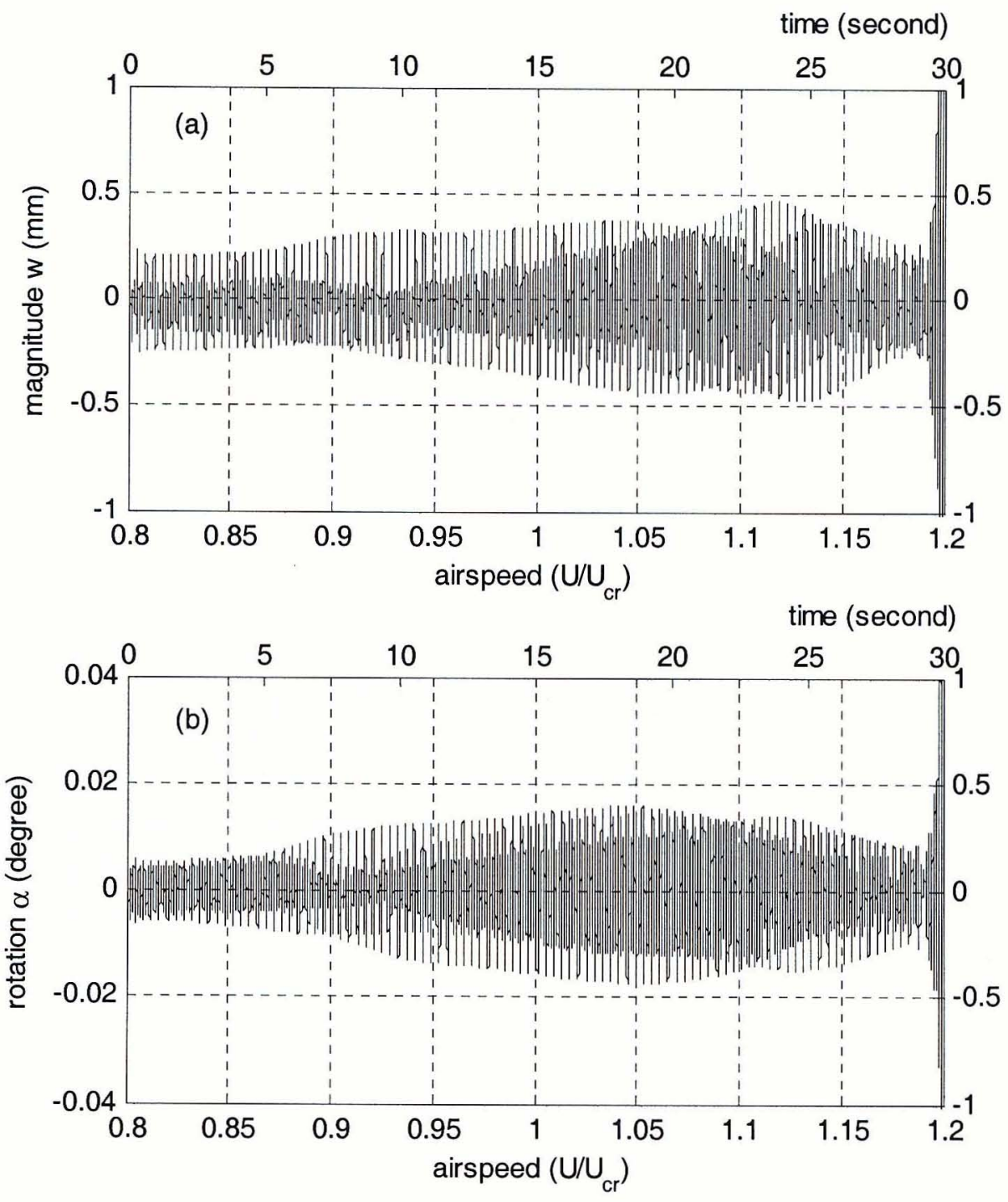

Fig. 6.17. Time responses of pitch-plunge airfoil with tunable DVA versus airspeed.

\subsection{Tunable DVA Design and Dynamic Analysis}

\subsubsection{Tunable DVA design and problem formulation}

From the results of previous analysis, the tunable DVA can increases the critical airspeed of the pitch-plunge airfoil to $1.141 U_{\mathrm{cr}}$. The problems of tunable DVA are there 
are two tunable components need to be controlled at the same time and the tunable stiffness component is not well developed yet. From Chapter 3, both stiffness and damping properties of system can be adjusted at the same time by tuning the damping coefficient of Maxwell element. The configuration of DVA with a tunable Maxwell element in system is designed and investigated in this section. The four-degree-offreedom analytical model of pitch-plunge airfoil with a tunable Maxwell element DVA is illustrated in Fig. 6.18.

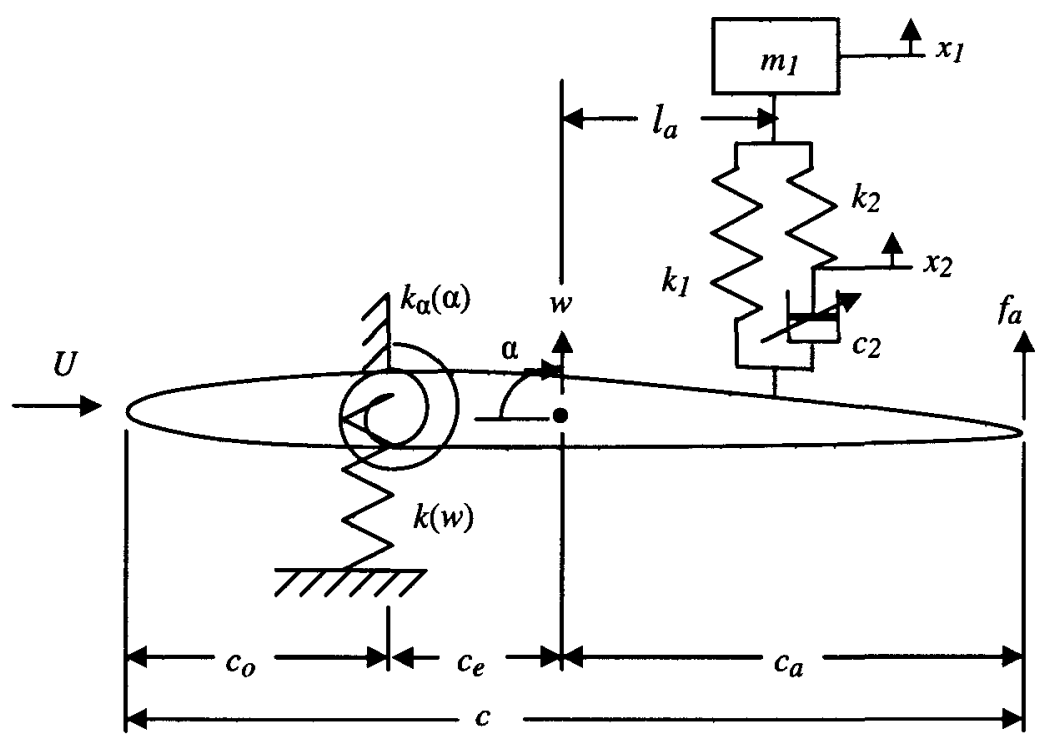

Fig. 6.18. Analytical model of pitch-plunge airfoil with absorber.

The governing equations of motion of the analytical model are,

$$
\mathbf{M} \ddot{\mathbf{x}}+(\mathbf{C}+U \mathbf{L}+\mathbf{D}) \dot{\mathbf{x}}+\left(\mathbf{K}+U^{2} \mathbf{H}+\mathbf{G}\right) \mathbf{x}=\mathbf{b} f_{a}
$$

where 


$$
\begin{aligned}
& \mathbf{x}=\left[\begin{array}{c}
w \\
\alpha \\
x_{1} \\
x_{2}
\end{array}\right], \quad \mathbf{b}=\left[\begin{array}{c}
1 \\
-c_{a} \\
0 \\
0
\end{array}\right], \quad \mathbf{M}=\left[\begin{array}{cccc}
m & 0 & 0 & 0 \\
0 & I & 0 & 0 \\
0 & 0 & m_{1} & 0 \\
0 & 0 & 0 & 0
\end{array}\right], \quad \mathbf{C}=\beta \mathbf{K}, \\
& \mathbf{K}=\left[\begin{array}{cccc}
k & -k c_{e} & 0 & 0 \\
-k c_{e} & k_{\alpha}+k c_{e}^{2} & 0 & 0 \\
0 & 0 & 0 & 0 \\
0 & 0 & 0 & 0
\end{array}\right], \quad \mathbf{H}=\frac{\rho c}{2} \frac{d C_{L}}{d \alpha}\left[\begin{array}{cccc}
0 & 1 & 0 & 0 \\
0 & c\left(\frac{1}{4}-\frac{c_{0}}{c}\right) & 0 & 0 \\
0 & 0 & 0 & 0 \\
0 & 0 & 0 & 0
\end{array}\right] \text {, } \\
& \mathbf{L}=\frac{\rho c}{2} \frac{d C_{L}}{d \alpha}\left[\begin{array}{ccccc}
1 & c\left(\frac{3}{4}-\frac{c_{0}}{c}\right) & 0 & 0 \\
c\left(\frac{1}{4}-\frac{c_{0}}{c}\right) & c^{2}\left[\left(\frac{1}{4}-\frac{c_{0}}{c}\right)\left(\frac{3}{4}-\frac{c_{0}}{c}\right)+\frac{\pi}{8 \frac{d C_{L}}{d \alpha}}\right] & 0 & 0 \\
0 & 0 & 0 & 0 \\
0 & 0 & 0 & 0
\end{array}\right] \\
& \mathbf{D}=\left[\begin{array}{cccc}
c_{1}+c_{2} & -a\left(c_{1}+c_{2}\right) & -c_{1} & -c_{2} \\
-a\left(c_{1}+c_{2}\right) & a^{2}\left(c_{1}+c_{2}\right) & a c_{1} & a c_{2} \\
-c_{1} & a c_{1} & c_{1} & 0 \\
-c_{2} & a c_{2} & 0 & c_{2}
\end{array}\right], \quad \mathbf{G}=\left[\begin{array}{cccc}
k_{1} & -a k_{1} & -k_{1} & 0 \\
-a k_{1} & a^{2} k_{1} & a k_{1} & 0 \\
-k_{1} & a k_{1} & k_{1}+k_{2} & -k_{2} \\
0 & 0 & -k_{2} & k_{2}
\end{array}\right],
\end{aligned}
$$

Equation (6.14) can also be written in state-space form as Eq. (6.5), but the matrix $\mathbf{A}$ and vectors $\mathbf{y}$ and $\mathbf{Q}$ are different from Eq. (6.6). For this analytical model, 


$$
\begin{aligned}
& \mathrm{A}=\left[\begin{array}{ccccccc}
0 & 0 & 0 & 0 & 1 & 0 & 0 \\
0 & 0 & 0 & 0 & 0 & 1 & 0 \\
0 & 0 & 0 & 0 & 0 & 0 & 1 \\
0 & 0 & \frac{k_{2}}{c_{2}} & -\frac{k_{2}}{c_{2}} & 1 & -l_{a} & 0 \\
\frac{R_{14} S_{41}}{m R_{44}}-\frac{S_{11}}{m} & \frac{R_{14} S_{42}}{m R_{44}}-\frac{S_{12}}{m} & \frac{R_{14} S_{43}}{m R_{44}}-\frac{S_{13}}{m} & \frac{R_{14} S_{44}}{m R_{44}}-\frac{S_{14}}{m} & \frac{R_{14} R_{41}}{m R_{44}}-\frac{R_{11}}{m} & \frac{R_{14} R_{42}}{m R_{44}}-\frac{R_{12}}{m} & \frac{R_{14} R_{43}}{m R_{44}}-\frac{R_{13}}{m} \\
\frac{R_{24} S_{41}}{I R_{44}}-\frac{S_{21}}{I} & \frac{R_{24} S_{42}}{I R_{44}}-\frac{S_{22}}{I} & \frac{R_{24} S_{43}}{I R_{44}}-\frac{S_{23}}{I} & \frac{R_{24} S_{44}}{I R_{44}}-\frac{S_{24}}{I} \frac{R_{24} R_{41}}{I R_{44}}-\frac{R_{21}}{I} \frac{R_{24} R_{42}}{I R_{44}}-\frac{R_{22}}{I} \frac{R_{24} R_{43}}{I R_{44}}-\frac{R_{23}}{I} \\
\frac{R_{34} S_{41}}{m_{1} R_{44}}-\frac{S_{31}}{m_{1}} & \frac{R_{34} S_{42}}{m_{1} R_{44}}-\frac{S_{32}}{m_{1}} & \frac{R_{34} S_{43}}{m_{1} R_{44}}-\frac{S_{33}}{m_{1}} & \frac{R_{34} S_{44}}{m_{1} R_{44}}-\frac{S_{34}}{m_{1}} & \frac{R_{34} R_{41}}{m_{1} R_{44}}-\frac{R_{31}}{m_{1}} & \frac{R_{34} R_{42}}{m_{1} R_{44}}-\frac{R_{32}}{m_{1}} \frac{R_{34} R_{43}}{m_{1} R_{44}}-\frac{R_{33}}{m_{1}}
\end{array}\right] \\
& \mathbf{y}=\left[\begin{array}{c}
w \\
\alpha \\
x_{1} \\
x_{2} \\
\dot{w} \\
\dot{\alpha} \\
\dot{x}_{1}
\end{array}\right], \quad \mathbf{Q}=\left[\begin{array}{c}
0 \\
0 \\
0 \\
0 \\
\frac{f_{a}}{m} \\
-\frac{c_{a} f_{a}}{I} \\
0
\end{array}\right], \\
& R_{i j}=C_{i j}+U L_{i j}+D, \quad S_{i j}=K_{i j}+U^{2} H_{i j}+G_{i j}
\end{aligned}
$$

The modal analysis is based on the eigenvalue problem of system matrix $\mathbf{A}$ in Eq.

(6.16). The time response analysis has the same equation as in Eq. (6.7-6.9). The matrix $A$ and vectors $\mathbf{y}$ and $\mathbf{Q}$ are based on Eq. (6.16).

\subsubsection{Optimized parameters of tunable Maxwell element DVA}

The primary stiffness $k_{1}$, and another stiffness $k_{2}$ are designed based on the stiffness values in Fig. 6.12. Tuning $c_{2}$ can match the stiffness or the damping curves in Fig. 6.12, but is difficult to match both of them at the same time. Usually, as the tunable damping $c_{2}$ increases, the system stiffness will increases and the system damping will decreases. The stiffness of DVA changes between $k_{1}$ and $k_{1}+k_{2}$. In order to tune the stiffness of the tunable Maxwell element DVA to fit the curve of stiffness in Fig. 6.12, $k_{1}$ should be smaller than the minimum stiffness value in Fig. 6.12 and the sum of $k_{1}$ and $k_{2}$ should be larger than the maximum stiffness value in Fig. 6.12 . 
From the analysis of previous sections, the dynamic behavior of DVA is not very sensitive to the parameters of DVA in the cases of $U<U_{\mathrm{cr}}$. The design of tunable DVA is focused in the range of $U>U_{\mathrm{cr}}$. From Fig.6.12, the optimized damping decreases and the optimized stiffness increases as airspeed increases in the range of airspeed $U>U_{\mathrm{cr}}$. Based on the stiffness in this range, the minimum stiffness is $25 \mathrm{~N} / \mathrm{mm}$ and the maximum stiffness is $150 \mathrm{~N} / \mathrm{mm}$. So, $k_{1}$ is chosen equal to $25 \mathrm{~N} / \mathrm{mm}$ and $k_{2}$ is chosen equal to 150 $\mathrm{N} / \mathrm{mm}$. Let $U=0.5,1,1.06$ and $1.12 U_{\mathrm{cr}}$ separately, $c_{2}$ change from 0 to $2.5 \mathrm{~N}-\mathrm{s} / \mathrm{mm}$ continuously, the minimum damping ratios with different values of $c_{2}$ are shown in Fig. 6.19. From Fig. 6.19 , the optimized $c_{2}$ may be found where the minimum damping ratio reaches its maximum value for each airspeed condition. Change airspeed $U$ from 0 to $1.2 U_{\mathrm{cr}}$ continuously, the optimized damping ratios and optimized value of $c_{2}$ are shown in Fig. 6.20. The critical airspeed $U$ is $1.065 U_{\text {cr }}$ and much lower than the result of tunable DVA in previous section where the critical airspeed $U$ is $1.141 U_{\mathrm{cr}}$. All of the natural frequencies and damping ratios of the system are shown in Fig. 6.21. 


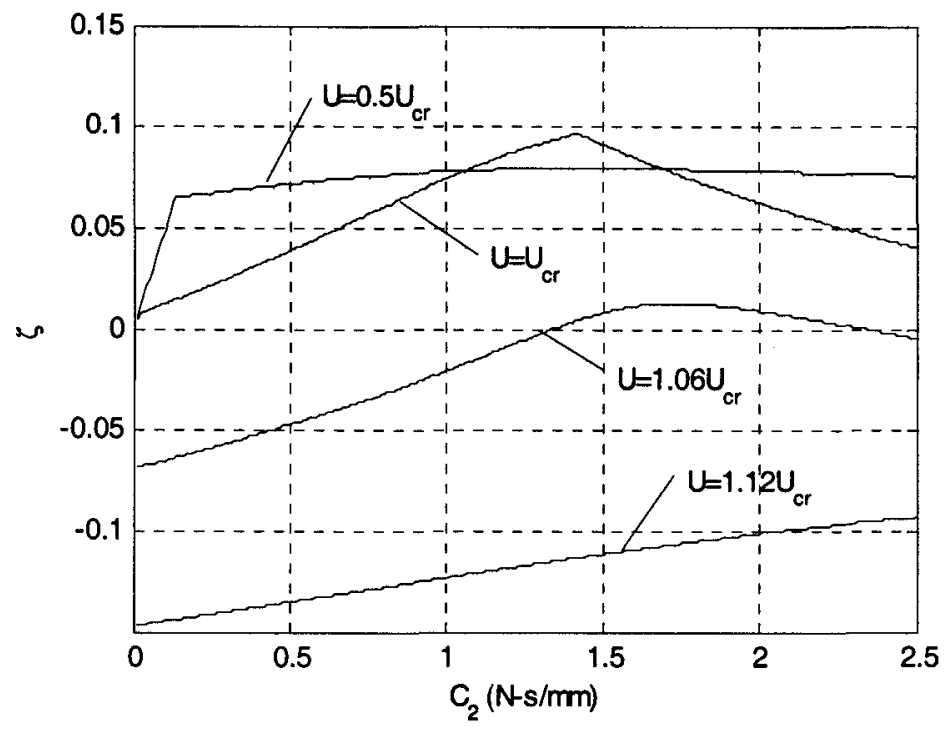

Fig. 6.19. The variation of minimum damping ratios as $c_{2}$ changes.
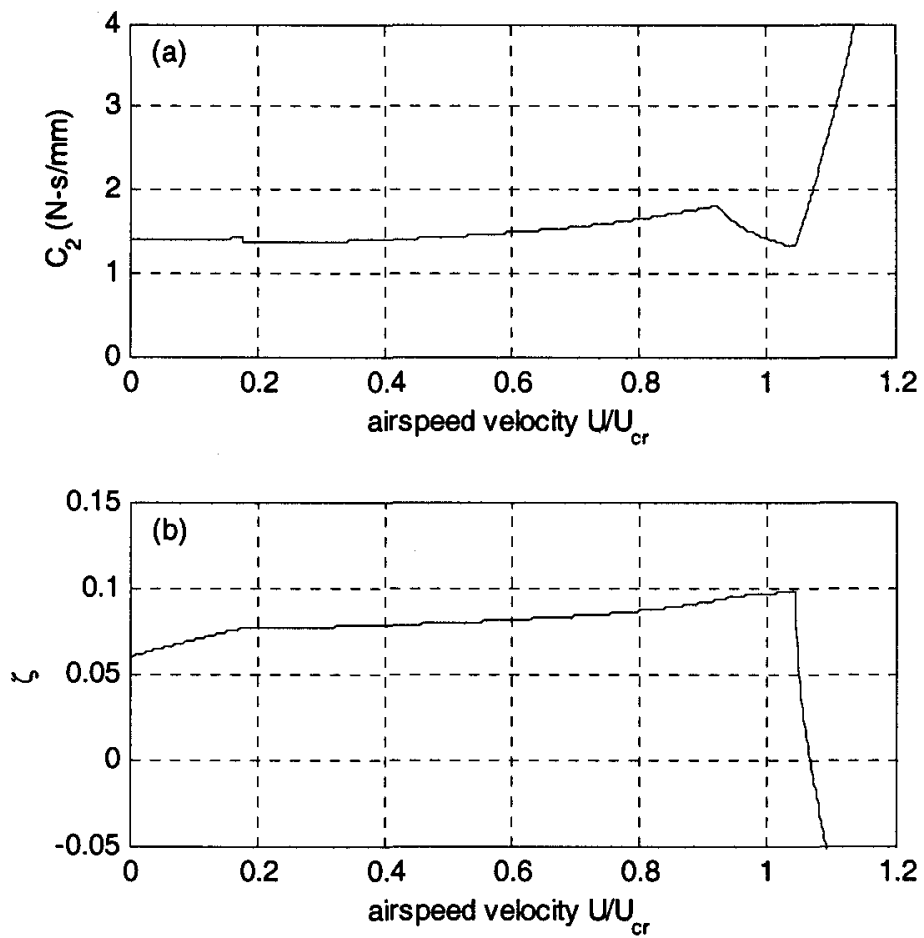

Fig. 6.20. Optimized Minimum damping ratios and associated $c_{2}$ at different airspeed. 

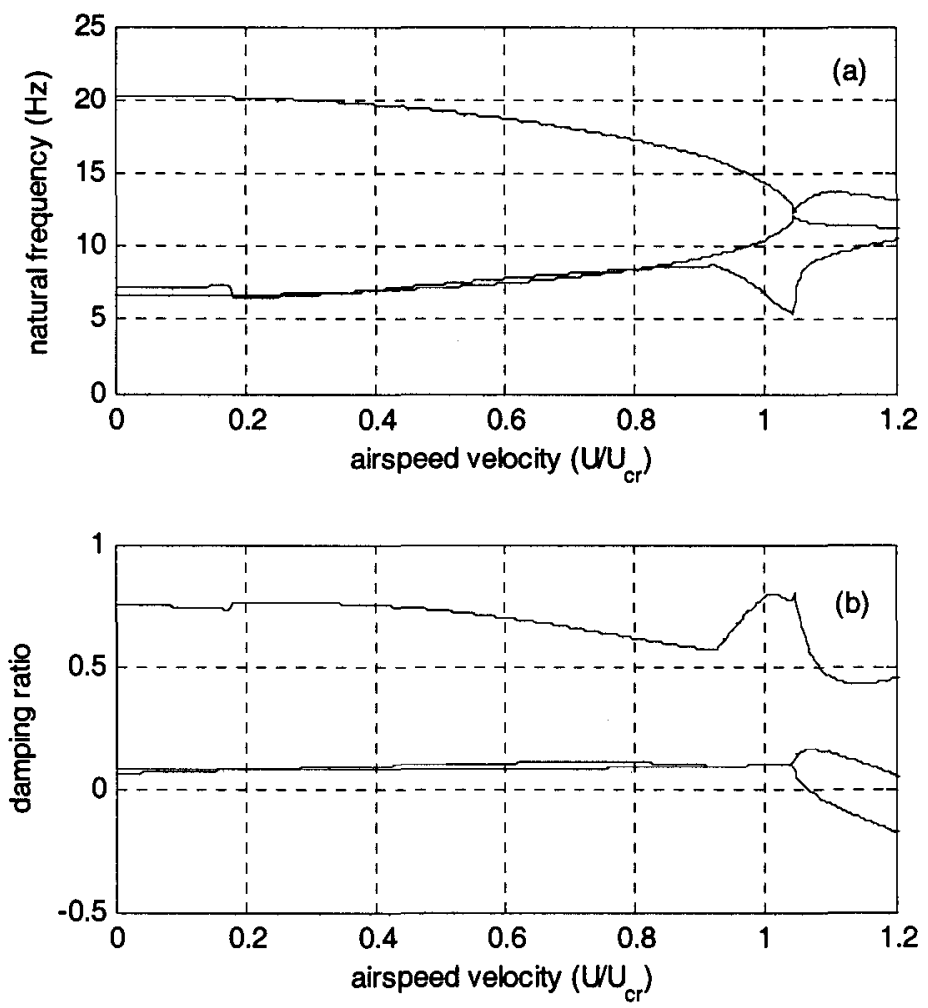

Fig. 6.21. Natural frequencies and damping ratios of tunable Maxwell element DVA.

Because the optimized damping and stiffness curves in Fig. 6.12 can not be very accurately matched at the same time by tuning $c_{2}$ in the range from airspeed $U=1$ to 1.2 $U_{\mathrm{cr}}$, the behavior of this tunable Maxwell element DVA is not as good as previous tunable DVA which has both tunable damping and stiffness components. From previous analysis, the vibration suppression behavior in the airspeed range from 1 to $1.06 U_{\mathrm{cr}}$ is not very sensitive to the value of tunable parameters as in the cases where airspeed $U>$ $1.06 U_{\mathrm{cr}}$. Another group of parameters of tunable DVA are designed based on the optimized stiffness and damping curves in Fig. 6.12 in the range of airspeed $U>1.06 U_{\mathrm{cr}}$. The primary stiffness $k_{1}$ is chosen equal to $90 \mathrm{~N} / \mathrm{mm}$ and the second stiffness $k_{2}$ is chosen equal to $70 \mathrm{~N} / \mathrm{mm}$. The adjustable range is smaller than the first tunable Maxwell 
element DVA, but is will perform better in the range $U>1.06 U_{\text {cr. }}$ Let $U=0.5,1,1.06$ and $1.12 U_{\mathrm{cr}}, c_{2}$ change from 0 to $1.2 \mathrm{~N}$-s/mm, the minimum damping ratios with different values of $c_{2}$ are shown in Fig. 6.22. From Fig. 6.22, the optimized $c_{2}$ exists where the damping ratio reaches its maximum value they are much better than the previous design which are shown in Fig. 6.19 in the cases of $U=1.06$ and $1.12 U_{\text {cr. }}$ Changing airspeed $U$ from 0 to $1.2 U_{\mathrm{cr}}$, the minimum damping ratios and optimized value of $c_{2}$ are shown in Fig. 6.23. The critical airspeed is $1.149 U_{\mathrm{cr}}$. This is even better than the result of tunable DVA which has both tunable stiffness and damping components where the critical airspeed is $1.141 U_{\mathrm{cr}}$. This tunable Maxwell element DVA will not improve as previous tunable in the range $U<1.06 U_{\mathrm{cr}}$ because it is designed based on the properties of system $U>1.06 U_{\mathrm{cr}}$. All of the natural frequencies and damping ratios of the system are shown in Fig. 6.24.

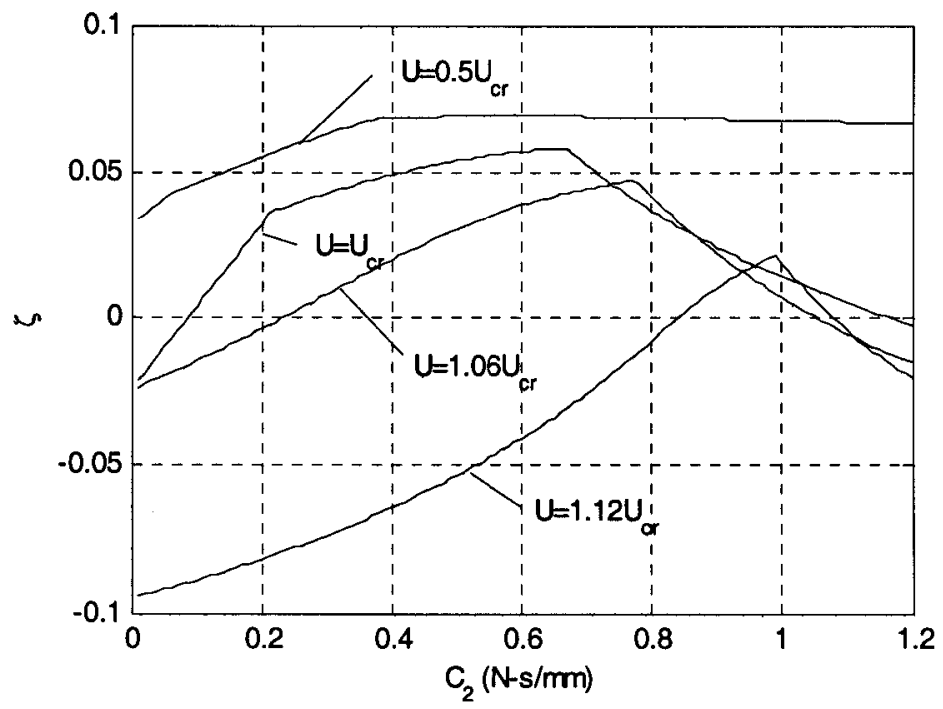

Fig. 6.22. The variation of minimum damping ratios as $c_{2}$ changes. 

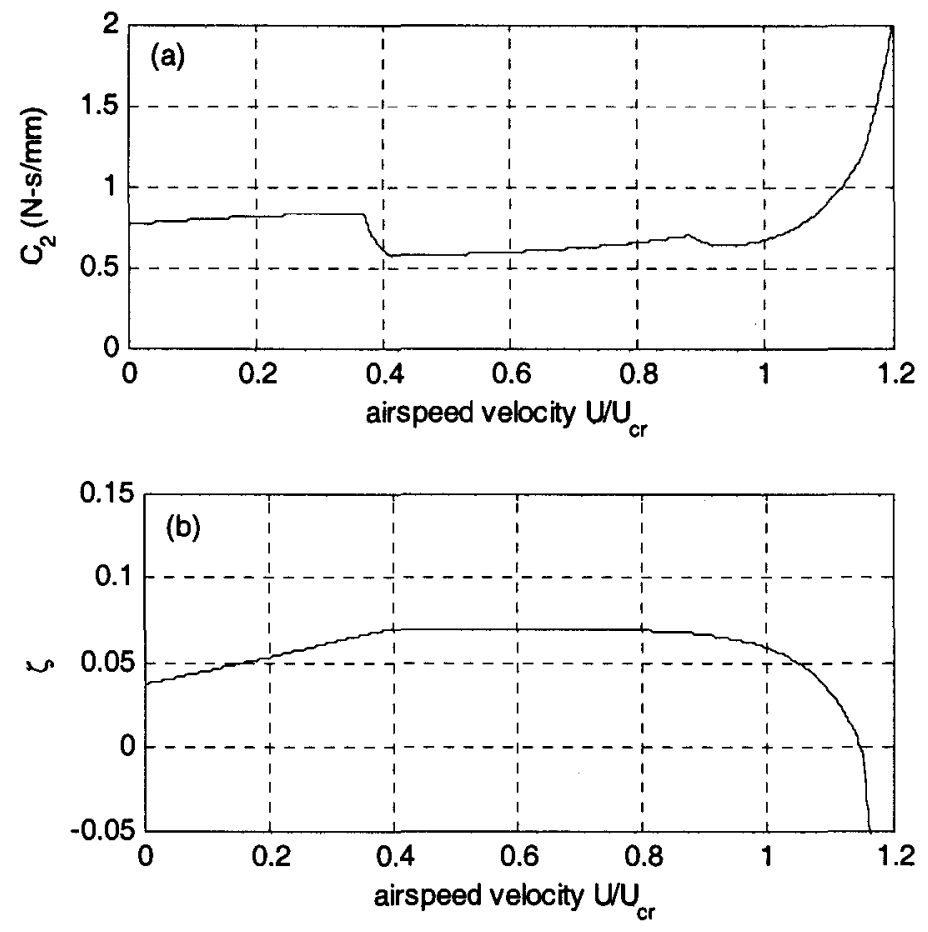

Fig. 6.23. Optimized Minimum damping ratios and associated $c_{2}$ at different airspeed. 

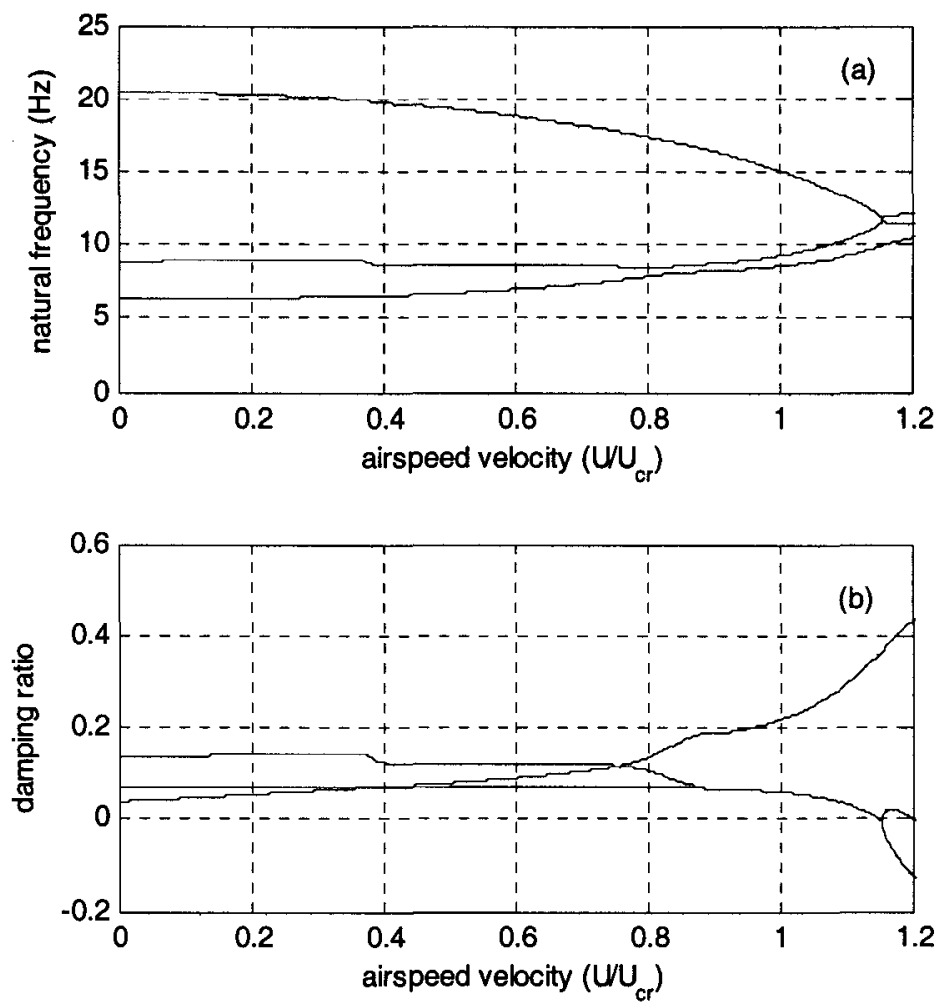

Fig. 6.24. Natural frequency and damping ratio of tunable Maxwell element DVA.

\subsubsection{Simulation results}

The time response of the pitch-plunge airfoil under random external excitation is simulated with airspeed accelerating continuously from $U / U_{\mathrm{cr}}=0.8$ to 1.2 at a linear rate for 30 seconds. The external excitation $f_{\mathrm{a}}$ with mean zero and standard deviation $1 \mathrm{kN}$ is applied over the 30 second time period. Results of first tunable Maxwell DVA are shown in Fig. 25 where the translational displacement $w$ and the rotational movement $\alpha$ are stable at the cases of the airspeeds under $U=1.1 U_{\mathrm{cr}}$, but increase very quickly after the airspeed reaches $U>1.11 U_{\text {cr. }}$ Results of second tunable Maxwell DVA are shown in Fig. 26 where the translational displacement $w$ and the rotational movement $\alpha$ are stable 
at the cases of the airspeeds under $U=1.17 U_{\mathrm{cr}}$, but increase very quickly after the airspeed reaches $U>1.17 U_{\mathrm{cr}}$.
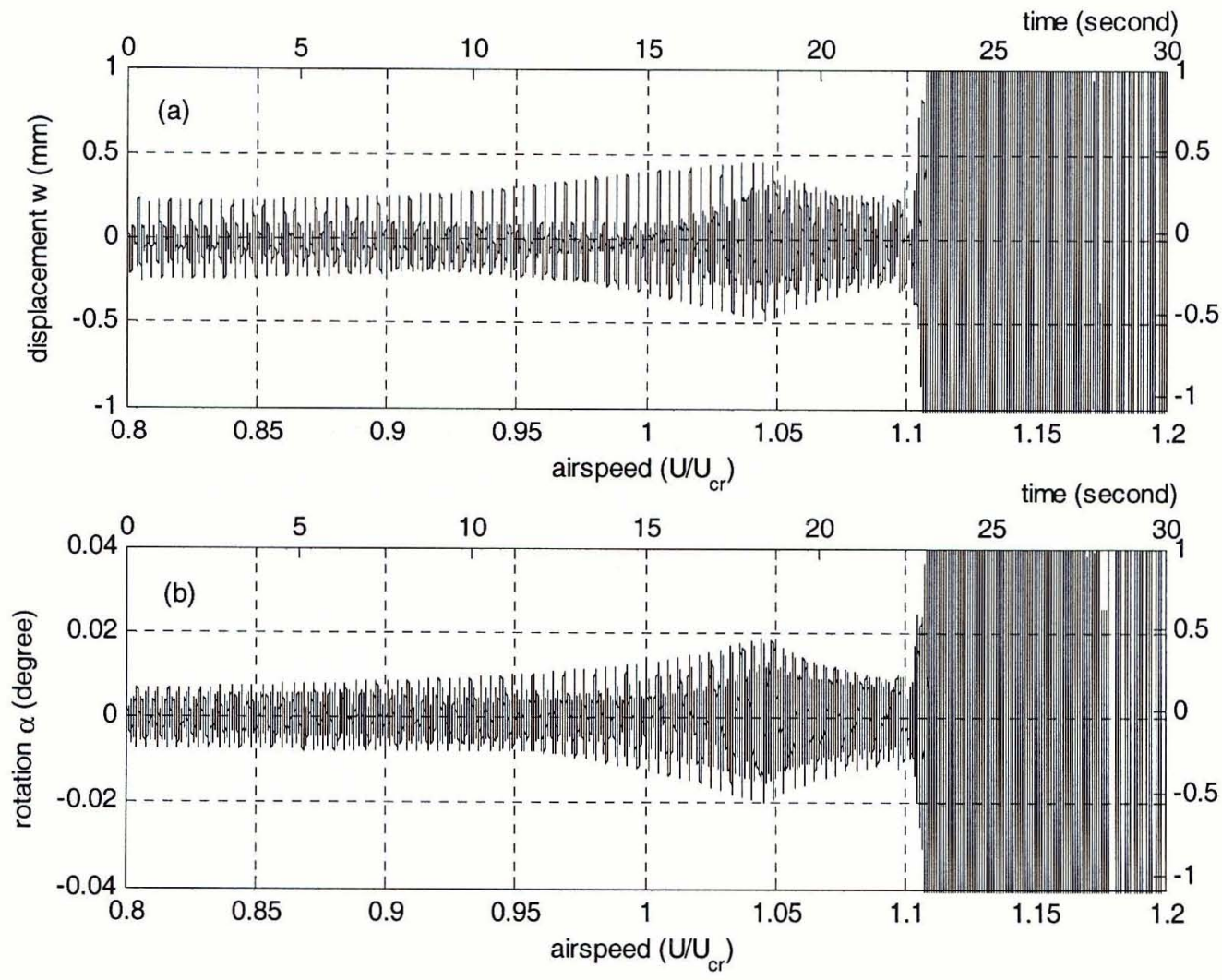

Fig. 6.25. Time responses simulation of pitch-plunge airfoil with first tunable Maxwell element DVA. 

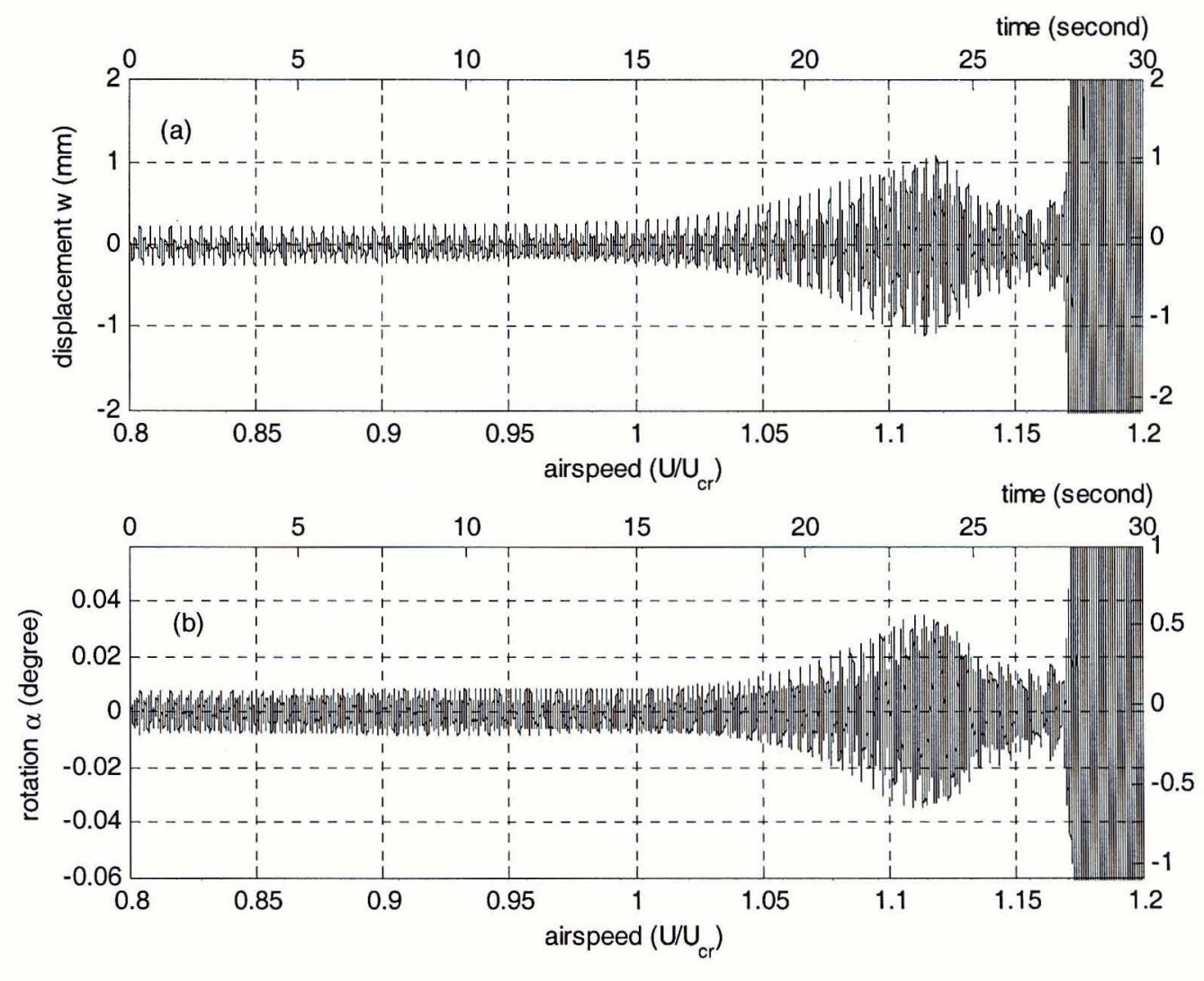

Fig. 6.26. Time responses of pitch-plunge airfoil with second tunable Maxwell element DVA.

\subsection{Summary}

In this chapter, the dynamic analysis reveals that the dynamic properties of aeroelastic aircraft wing such as natural frequencies and damping ratios change as airspeed changes. The aeroelastic aircraft wing turns to be unstable at high airspeed condition. The DVA can improve the dynamic behavior at high airspeed condition. Further investigation reveals that the DVA with tunable stiffness and damping components performs better than the constant parameter DVA. Because the tunable stiffness component is not well developed yet, an alternative configuration, tunable 
Maxwell element DVA, which has only one tunable damping component is designed.

Dynamic analysis and simulation reveal the performance of the tunable Maxwell element DVA close to the tunable DVA which has both tunable stiffness and damping components. 


\section{CHAPTER 7 \\ SEMI-ACTIVE VIBRATION CONTROL}

The feasibility of semi-active vibration control of aircraft wing with tunable Maxwell element DVA is discussed in previous chapter. The control methods of tunable Maxwell element DVA is investigated in this chapter. Two control methods are developed. The first control method is based on the measured airspeed signals. From Eq. (6.1-6.2), the airspeed is not the only factor which influences the dynamic properties of aeroelastic aircraft wing, other factors such as air density also have important influences. Due to some of these influence factors can not be accurately measured, the second control method is based on the vibration spectra signals of aeroelastic wing. The acceleration signals and their spectra are convenient to be measured and they reflect the influences of all disturbing factors. The relationship between the vibration spectra signals and control signals $\left(c_{2}\right)$ is established via neural network based model. Both control methods are based on the measured signals and do not require prior knowledge of the plant parameters.

\subsection{Control Method based on Measured Airspeed}

In previous chapter, the objective of vibration control is based on system stability or the minimum damping ratio. However, the damping ratio is not easy to be accurately 
measured online because the disturbance from aileron is difficult to be measured. In this chapter, the RMS (root mean square) of vibration time response is used as the objective of control.

\subsubsection{Objective of control}

The RMS of vibration time response changes as the parameter of tunable damper $c_{2}$ changes. The way of change is very similar as the minimum damping ratio changes. In the cases of the value of $c_{2}$ is the only variable parameter and all other parameters in the system are constants, the influences of the value of $c_{2}$ on RMS at different airspeed are investigated. Fig. 7.1-7.3 show how the RMS changes in cases of the airspeed $U=$ $0.5,1,1.06$ and $1.12 U_{\mathrm{cr}}$ as $c_{2}$ changes. Fig. 7-1 shows the RMS of vibration time responses at translational coordinate. Fig. 7-2 shows the RMS of vibration time responses at rotational coordinate. The optimized $c_{2}$ for the RMS of vibration time responses at translational and rotational coordinates are different based on Fig. 7-1 and Fig. 7-2. The optimized values of $c_{2}$ can be either one of them or a kind of weighted sum. 


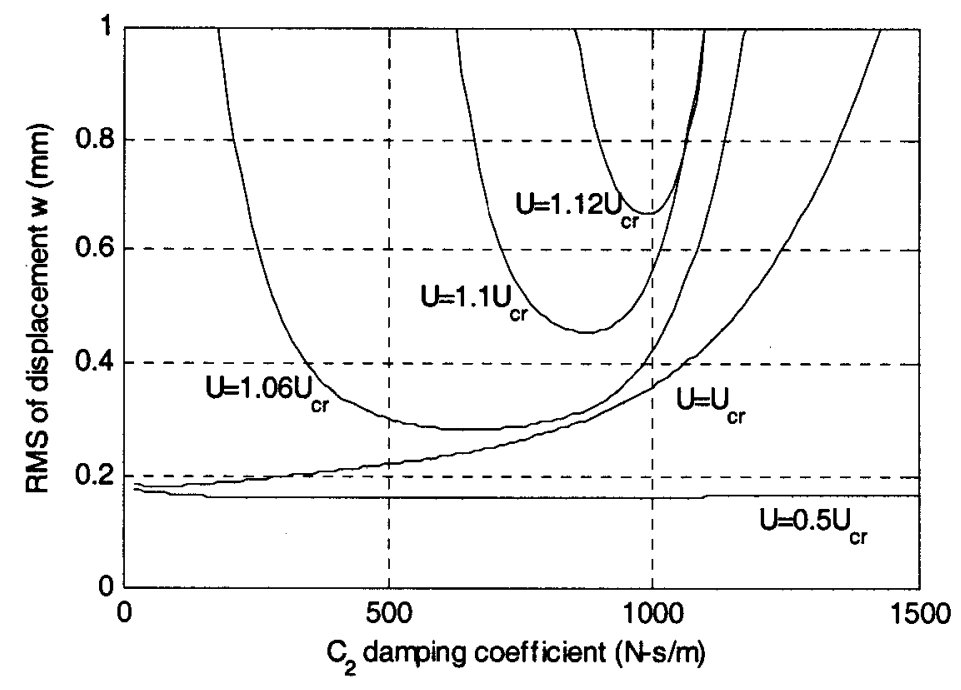

Fig. 7.1. Translational RMS of vibration time responses changes as the values of $c_{2}$ changes at different airspeed.

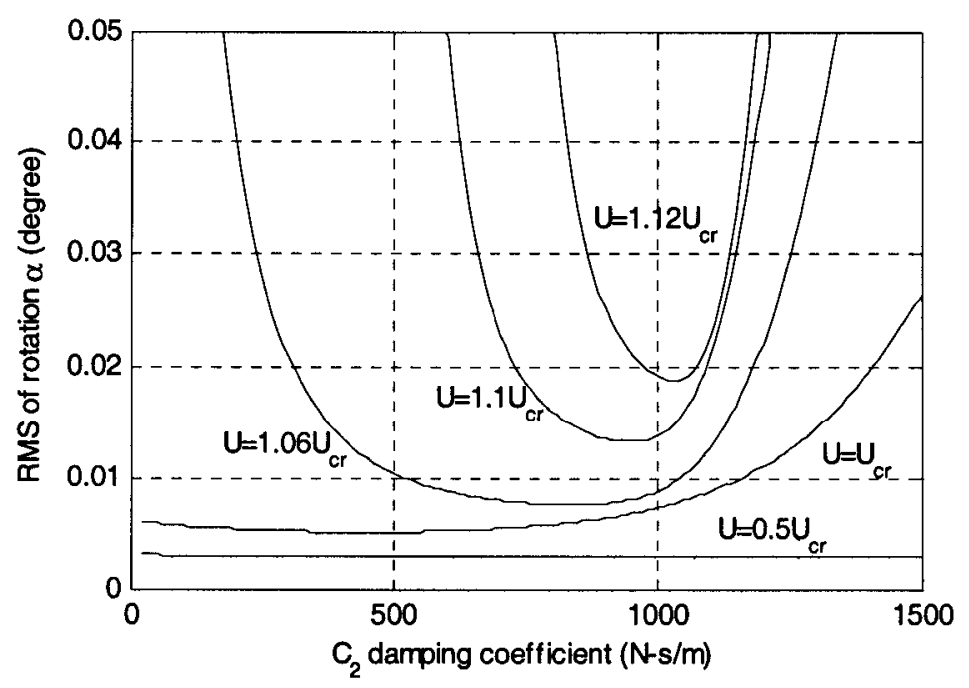

Fig. 7.2. Rotational RMS of vibration responses changes as the values of $c_{2}$ changes at different airspeed.

In this study, the RMS of vibration response at the leading edge is used as the objective of control. Because it is a kind of weighted sum of the RMSs of vibration 
response at two different degrees and it is easy to be measured than vibration response at rotational degree. Fig. 7-3 shows how the RMS vibration responses at leading edge changes as the value of $c_{2}$ changes at different airspeed.

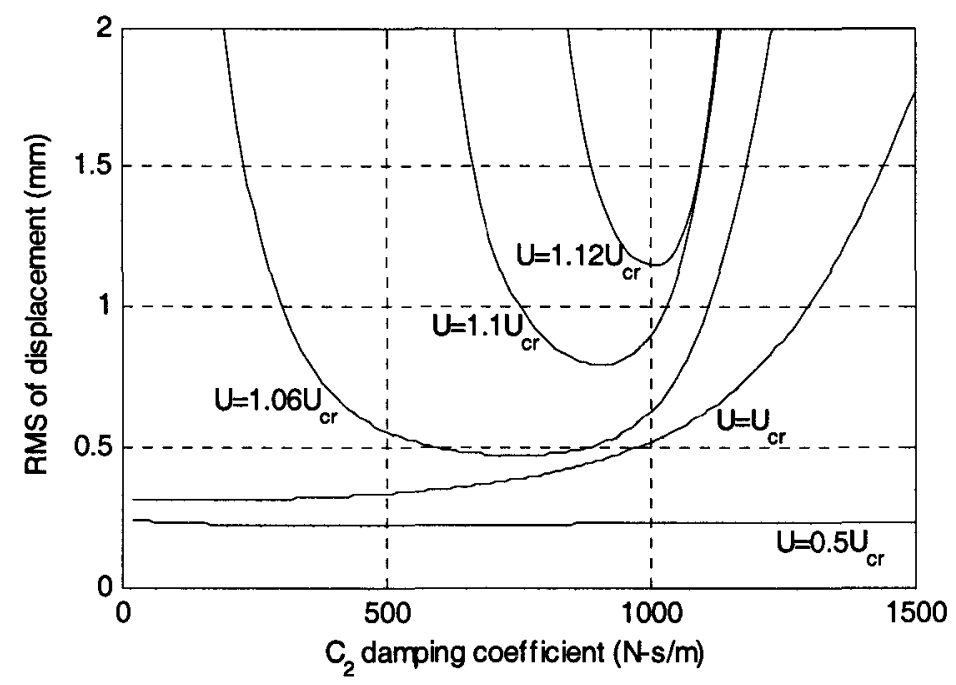

Fig. 7.3. Vibration responses RMS at leading edge change as the values of $c_{2}$ changes at different airspeeds.

From Fig. 7.1-7.3, all the values of RMS of vibration time responses are small in case of the airspeed $U=0.5 U_{\mathrm{cr}}$. The values of RMS are not very sensitive to the changes of the value of $c_{2}$. As airspeed increases, the RMS of vibration responses increases. For any given airspeed, there is an optimized value of $c_{2}$ where the RMS has the minimum value. The optimized $c_{2}$ and associated minimum RMS of vibration time responses at the airspeed from 0.8 to $1.2 U_{\mathrm{cr}}$ are shown in Fig. 7-4. In practice, this optimized $c_{2}$ may be obtained by tuning its value in experiment. If the airspeed is higher than $1.14 U_{\mathrm{cr}}$, the RMS of vibration responses increases very quickly because the system turns to be 
unstable. The system will be broken if experiment does not stop at the airspeed $U=$ $1.14 U_{\mathrm{cr}}$.
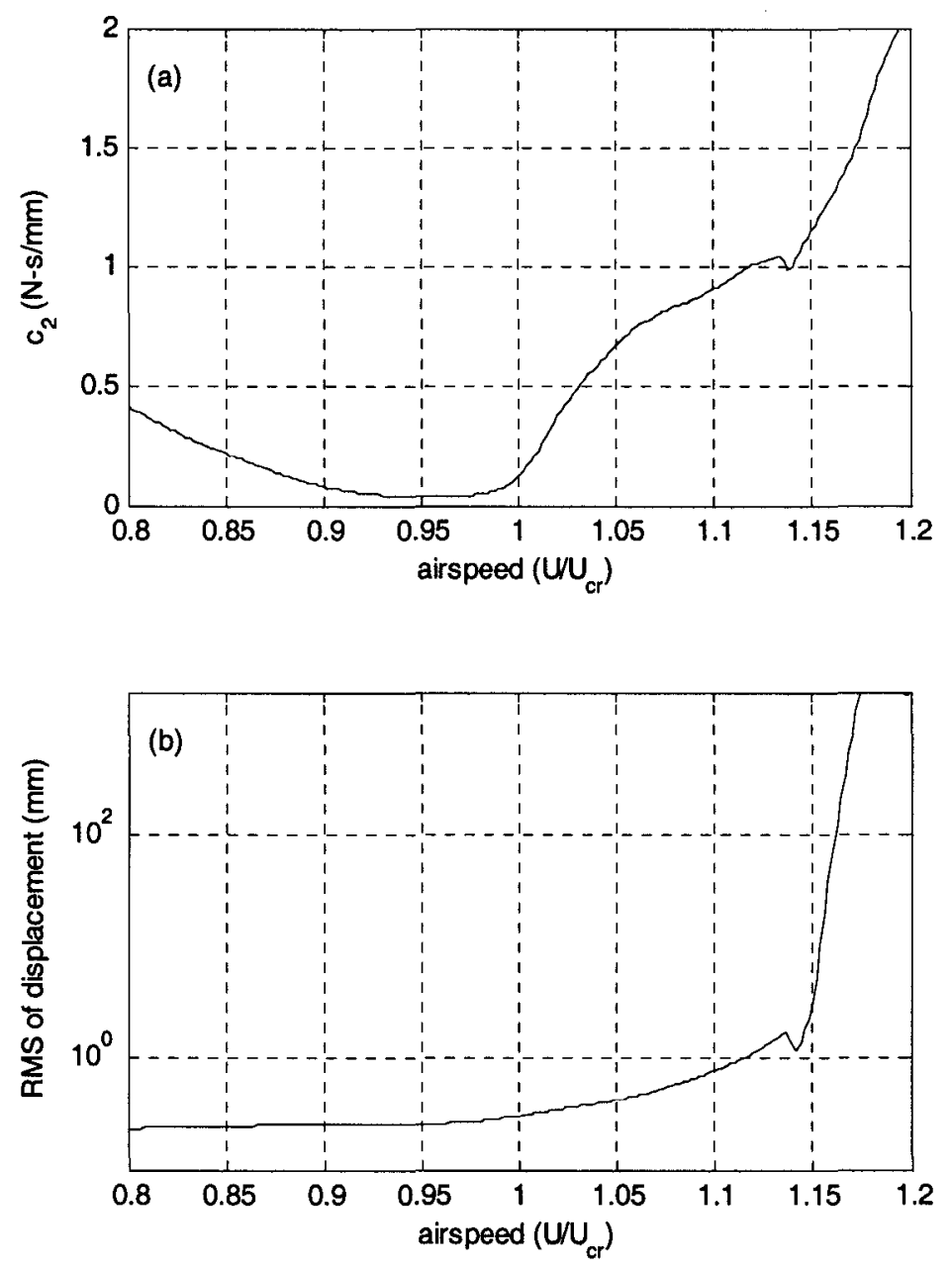

Fig. 7.4. Optimized damping coefficients and the RMS of vibration time response at leading edge. 
Because the curve in Fig. 7.4 is almost linear in the range of $U>1.14 U_{\mathrm{cr}}$, the optimized $c_{2}$ can be calculated use least square method based on the data from $U=115$ to $120 \mathrm{~m} / \mathrm{s}$.

$$
c_{2}=a_{0}+a_{1} U
$$

\subsubsection{Flight Simulation}

The time response of the pitch-plunge airfoil under random external excitation is simulated with airspeed accelerating continuously from $U=0.8$ to $1.2 U_{\mathrm{cr}}$ at a linear rate for 30 seconds. The external excitation $f_{\mathrm{a}}$ with mean zero and standard deviation $1 \mathrm{kN}$ is applied over the 30 second time period. Results of tunable Maxwell DVA which is controlled by the measured airspeed signals are shown in Fig. 7.5 where the translational displacement $w$ and the rotational movement $\alpha$ are stable at the cases of the airspeeds under $U=1.17 U_{\mathrm{cr}}$, but increase very quickly after the airspeed reaches $U>1.17 U_{\mathrm{cr}}$. The magnitude of objective in this chapter is much easier to be measured than the control objective used in Chapter 6 . 

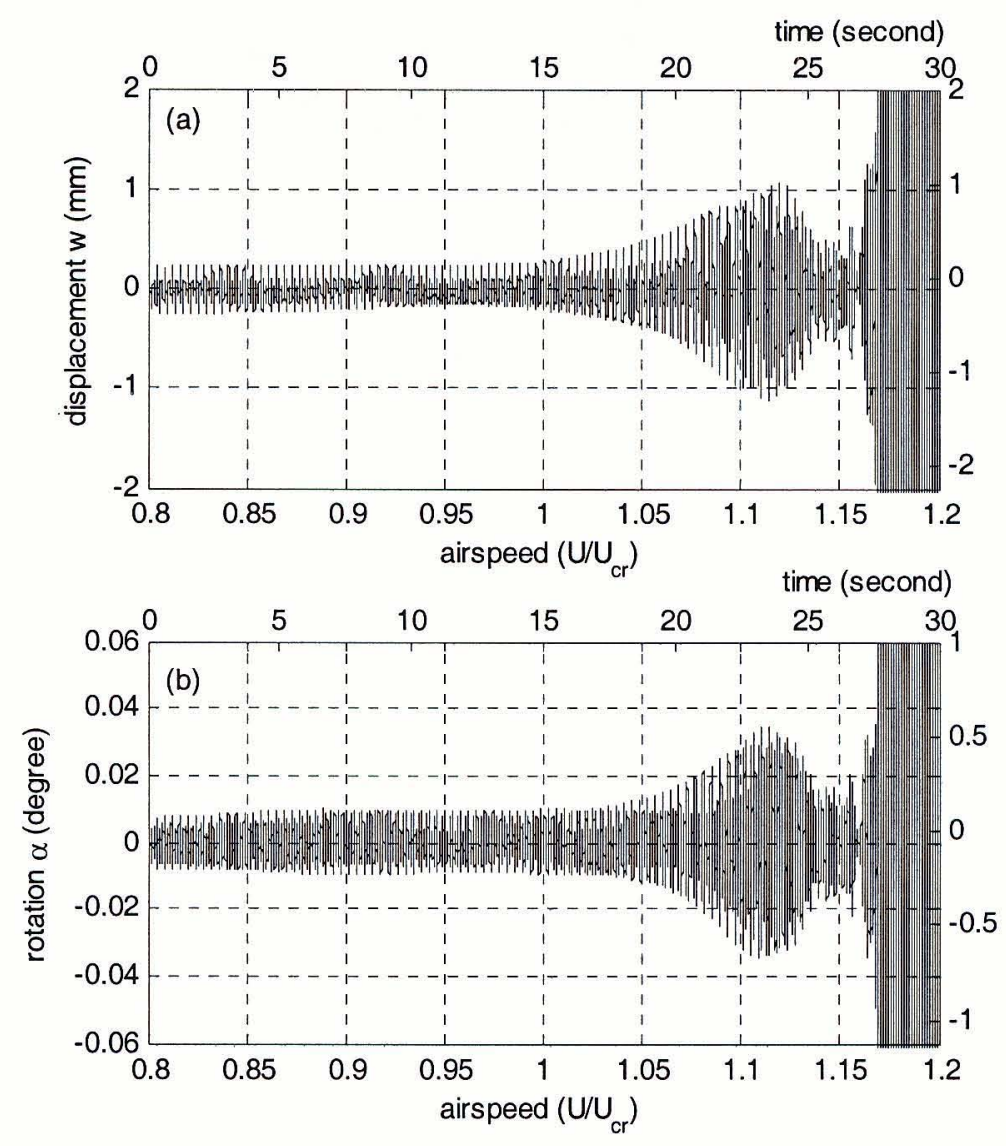

Fig. 7.5. Time responses of pitch-plunge airfoil with tunable Maxwell element DVA.

\subsubsection{Simulation with variable air density}

In practice, the airspeed is not the only variable factor during flight. Other parameters, such as air density, may also change and influence the dynamic properties. Assume air density $\rho$ changes as in $\mathrm{Eq}(7.2)$,

$$
\rho=\rho_{0}(1+\mu \sin (\omega t))
$$

Where $\rho_{0}=1.2, \mu=0.05,0.1 ; \omega=2 \pi$. Flying simulation results of vibration responses in the translational and rotational coordinates are shown in Fig. 7.6-7.7. Figure 7.6 shows the results with parameter $\mu=0.05$. Figure 7.7 shows the results with $\mu=0.1$. 

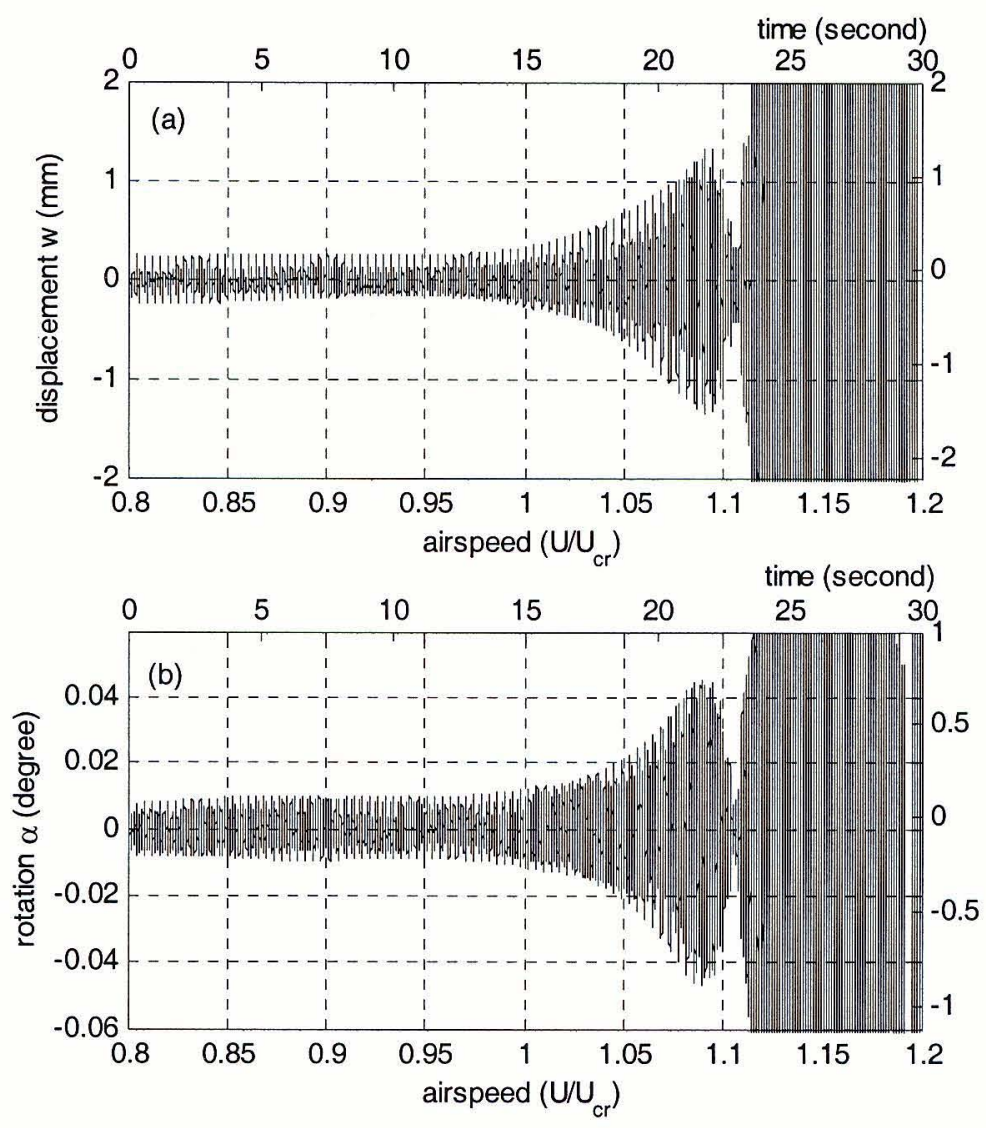

Fig. 7.6. Time responses of pitch-plunge airfoil with tunable Maxwell element DVA with

$$
\mu=0.05 \text {. }
$$



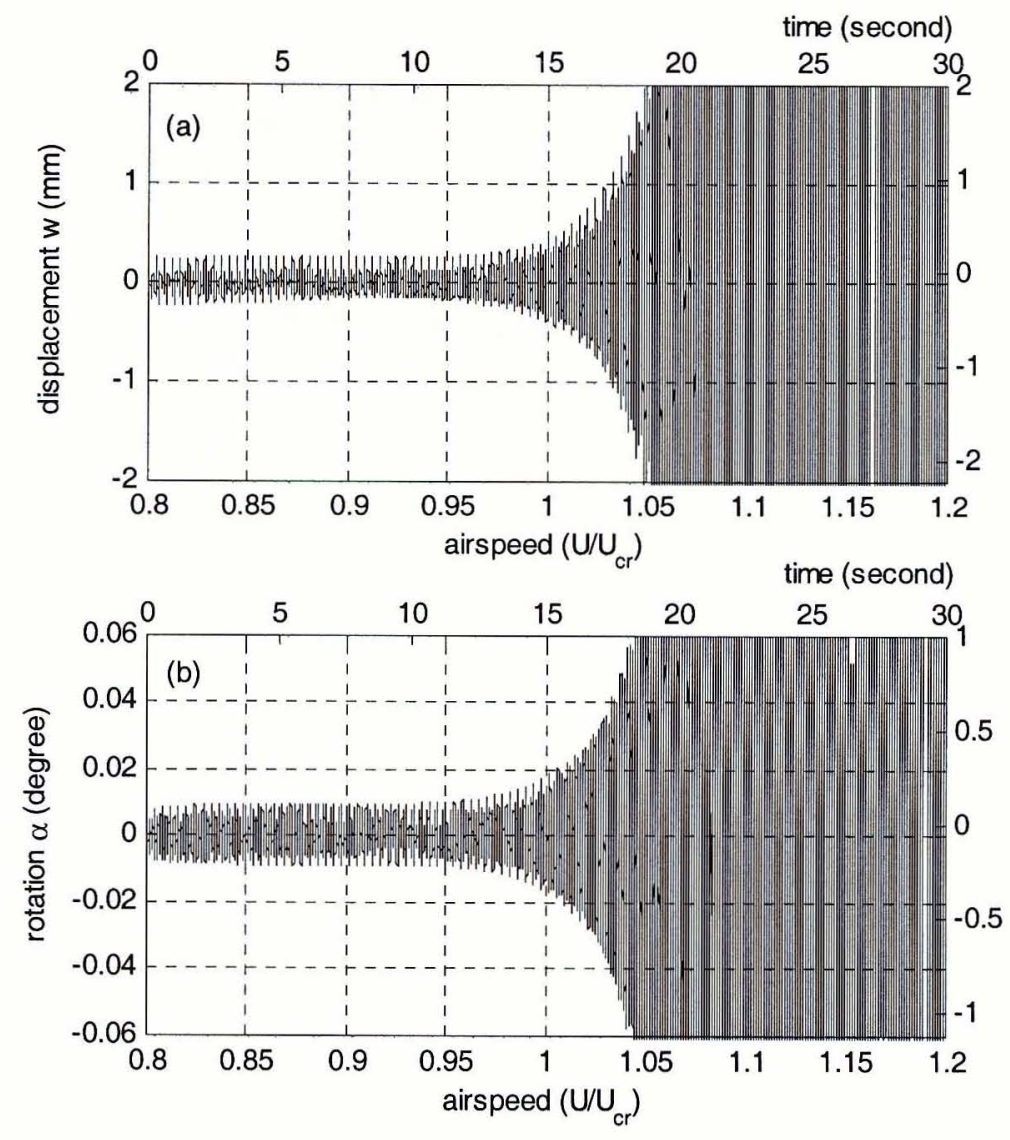

Fig. 7.7. Time responses of pitch-plunge airfoil with tunable Maxwell element DVA with

$$
\mu=0.1 \text {. }
$$

From Fig. 7.6, it is found that the system is unstable at the airspeed $U=1.11 U_{\text {cr }}$ in the case of $\mu=0.05$. This is not as good as the results shown in Fig. 7.5. In the case of $\mu$ $=0.1$, the system is unstable at the airspeed $U=1.04 U_{\text {cr }}$. This is close to the results from the system without DVA which is shown in Fig. 6.4. So, the control algorithm based on the airspeed can not work well in the case of air density varies significantly. It is necessary to develop new control method for the case where air density is variable during flight. 


\subsection{Control by Vibration Spectra Signals}

The scaled vibration spectra are the signals which reflect the system properties and external disturbance. All the changes on airspeed, air density and other external disturbances play influences on the scaled vibration spectra. The relationship between the vibration spectra and the optimized value of $c_{2}$ is very complicated. In this study, a neural network model is employed to establish this relationship. Two parts of works will be carried out, the system identification and system control with the neural network model.

\subsubsection{System identification}

In the system identification stage, a neural network model is identified for representing the relationship between the vibration spectra and optimized $c_{2}$. There is an optimized value of $c_{2}$ if all other flight parameters are constants during the process of tuning $c_{2}$. Based on the current value of $c_{2}$ and the measured vibration spectrum, the value of $c_{2}$ is tuned to find the optimized value using experimental method. The objective of optimization is the RMS of vibration time responses. The prediction error between the optimized output and the neural network model output is used as the neural network training signal. The process of system identification is illustrated by Fig. 7.8. 


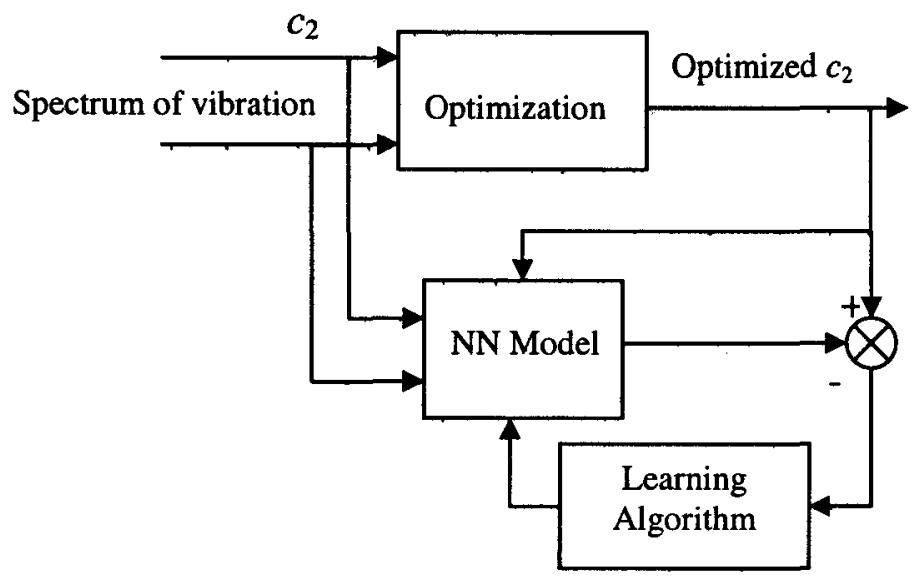

Fig. 7.8. Configuration of system identification process.

The design of neural network architecture may be pursued in a variety of ways such as feedforward backpropagation networks [44] or radial-basis networks [45]. Although the radial-basis networks require more neurons than standard feedforward backpropagation networks, they work better when many training signals are available. They can be designed in a fraction of the time it takes to train standard feedforward backpropagation networks.

The architecture of single radial-basis neuron with $R+1$ input is shown in Fig. 7.9.

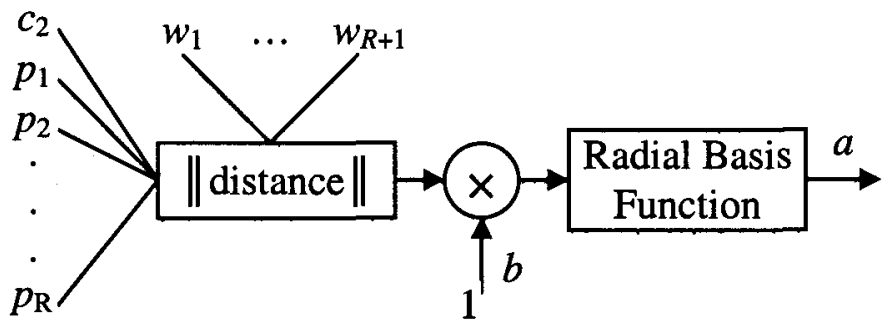

Fig. 7.9. Architecture of radial basis neuron. 
Where $p_{1}, \ldots, p_{R}$ represent the scaled spectrum magnitude of vibration responses at specific frequency. If the magnitude of vibration responses at specific frequency is $P_{\mathrm{i}}$, the scaled value of spectrum at specific frequency is

$$
p_{i}=\frac{P_{i}}{\sum_{i=1}^{R} P_{i}}
$$

The $w_{1}, \ldots, w_{\mathrm{R}+1}$ represent weight vector of neuron. The input to the transfer function of radial basis neuron is the vector distance between its weight vector $w$ and the input vector $\mathbf{p}$, multiplied by the bias $b$. The output is

$$
a=f(b\|\mathbf{w}-\mathbf{p}\|)
$$

The transfer function for radial basis neuron is

$$
f(n)=e^{-n^{2}}
$$

The radial basis function has a maximum of 1 when its input is 0 . As the vector distance between $\mathbf{w}$ and $\mathbf{p}$ increases, the value of output decreases. Thus, a radial basis neuron works as a detector to recognize how close the input $\mathbf{p}$ is to its weight vector $\mathbf{w}$. The bias $b$ allows the sensitivity of the neuron to be adjusted. The spread constant $n$ is used to control the influence of input vector based on the distant.

The radial basis network in this study consists of two layers: a hidden radial basis layer of $S_{1}$ neurons, and an output linear layer with only one neuron. The architecture of the radial basis network is shown in Fig. 7.10. 


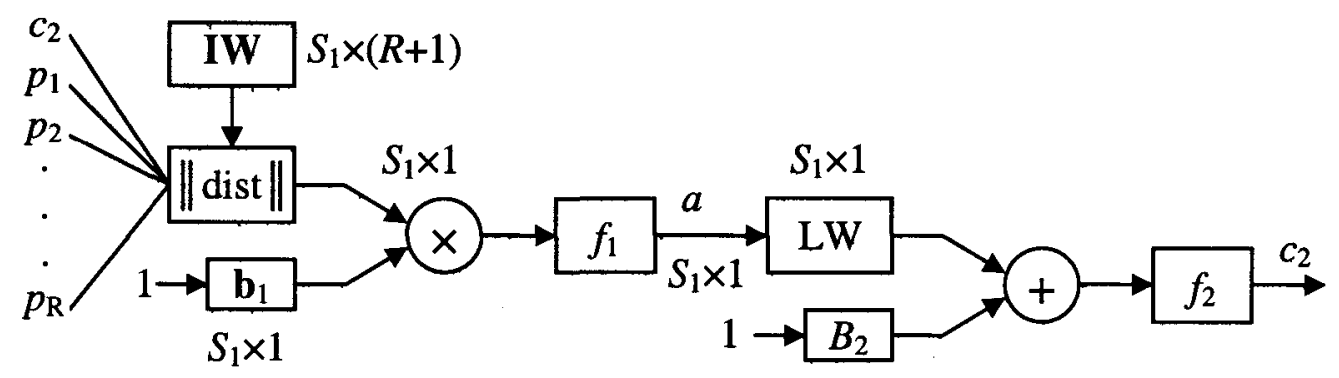

Fig. 7.10. Architecture of radial basis network.

The $\|$ dist $\|$ box in Fig. 7.10 accepts the input vector and the weight matrix IW, and produces a vector having $S_{1}$ elements. The bias vector $\mathbf{b}_{1}$ and the output of $\|$ dist $\|$ box are combined with the element by element multiplication. The output of first layer is the vector after transfer function of radial basis neuron. In the second layer, the operation includes the element by element multiplication of output vector of first layer and the weight vector $\mathbf{L W}$, the sum operation with bias $b_{2}$ and the linear transfer function $f_{2}$. The learning process undertaken by the radial basis network is referenced in [46]. In this study, the training data for the radial basis network model are from the experiment parameters airspeed $U$ from 0.8 to $1.14 U_{\text {cr }}$ with increments $0.002 U_{\text {cr. }}$. At each airspeed condition, 20 different values of $c_{2}$ and associated scaled vibration spectra are used as input data, the optimized values of $c_{2}$ at the airspeed are used as output data. Both movements on the translational and rotational degree are measured. The spectra values for train the radial basis network are from 5 to $20 \mathrm{~Hz}$ with $1 \mathrm{~Hz}$ increment. Sampling frequency is $1,000 \mathrm{~Hz}$ and sampling time is 2 second. There are 33 input channels and 1 output channel in the radial basis neural network. Fig. 7.11 shows the optimized $c_{2}$ from experiment and the calculated results of $c_{2}$ from neural network model. From this figure, the difference is obvious in the low frequency range, but neglectable in the high 
frequency range because the different sensitivity of system output on the value of $c_{2}$ in different airspeed range. In the range where the output is sensitive to the value of $c_{2}$, the calculated results from radial basis neural network model is very close to the values of optimized $c_{2}$ from experimental simulation.

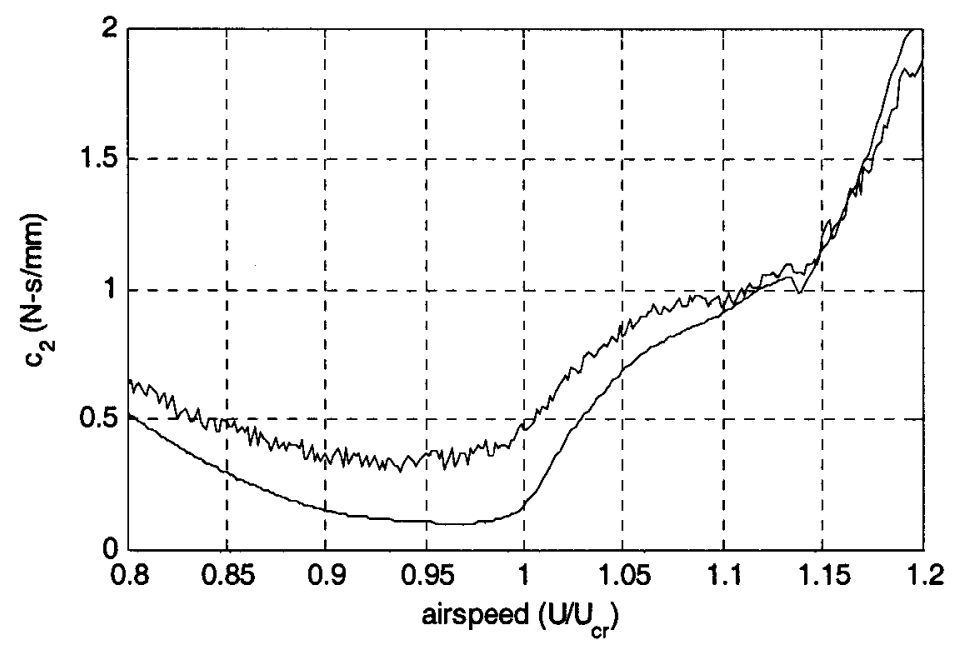

Fig. 7.11. Comparison of the value of $c_{2}$ from neural network model and optimized value of $c_{2}$ from experiment simulation.

\subsubsection{System control}

In the process of system vibration absorption control, the identified neural network model is employed for the control of the tunable Maxwell element DVA. The configuration of the control system is shown in Fig. 7.12. 


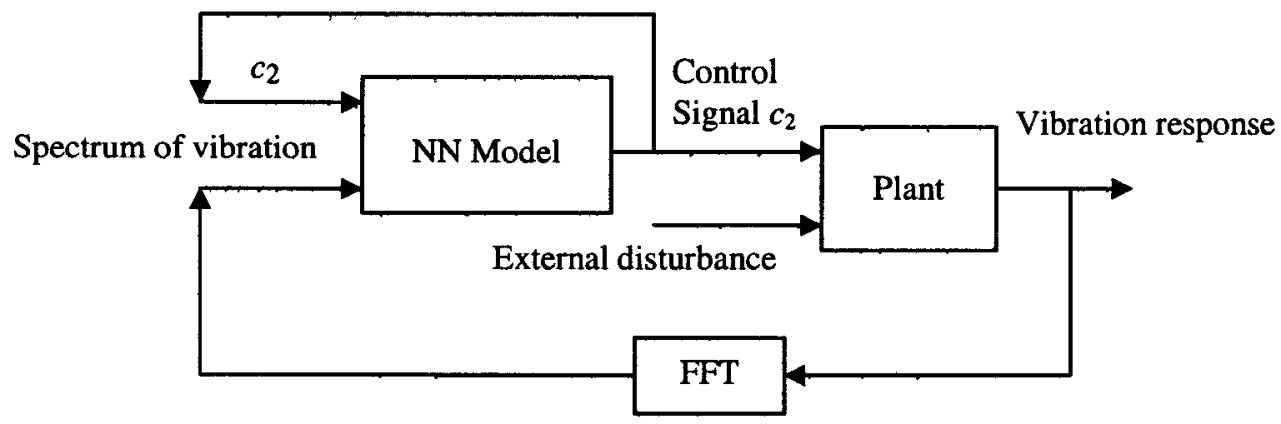

Fig. 7.12. Configuration of the control system with the neural-network based controller.

Based on the current value of $c_{2}$ and scaled measured vibration spectrum, the value of $c_{2}$ from neural network model is used as control signals to the tunable Maxwell element DVA. The FFT of measured scaled vibration response are used for the input signals of neural network model.

\subsubsection{Flight simulation}

The time response of the pitch-plunge airfoil under random external excitation is simulated with airspeed accelerating continuously from $U=0.8$ to $1.2 U_{\mathrm{cr}}$ at a linear rate for 30 seconds. The external excitation $f_{\mathrm{a}}$ with mean zero and standard deviation $1 \mathrm{kN}$ is applied over the 30 second time period. Results of tunable Maxwell DVA which is controlled based on scaled measured vibration spectra signals by the neural network model are shown in Fig. 7.13 where the translational displacement $w$ and the rotational movement $\alpha$ are stable at the cases of the airspeeds under $U=1.16 U_{\mathrm{cr}}$, but increase very quickly after the airspeed reaches $U>1.16 U_{\text {cr. }}$ This result is almost as good as the results in Fig. 7.5 where the control signals are based on airspeed and the responses 
increase quickly after the airspeed reaches $U=1.17 U_{\mathrm{cr}}$. The associated values of $c_{2}$ during the process of simulation for control are shown in Fig. 7.14.
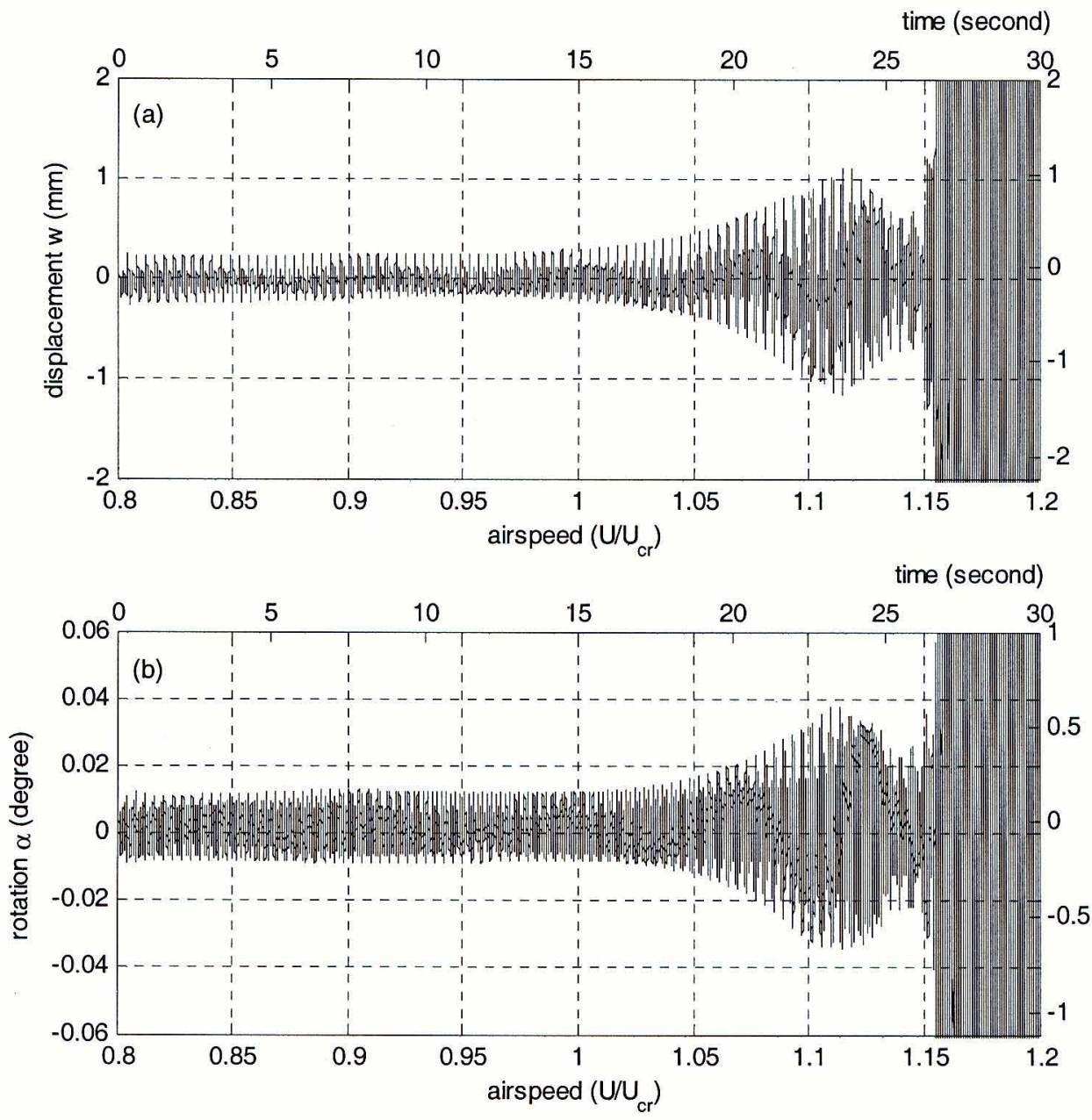

Fig. 7.13. Time responses of pitch-plunge airfoil with neural-network based controller. 


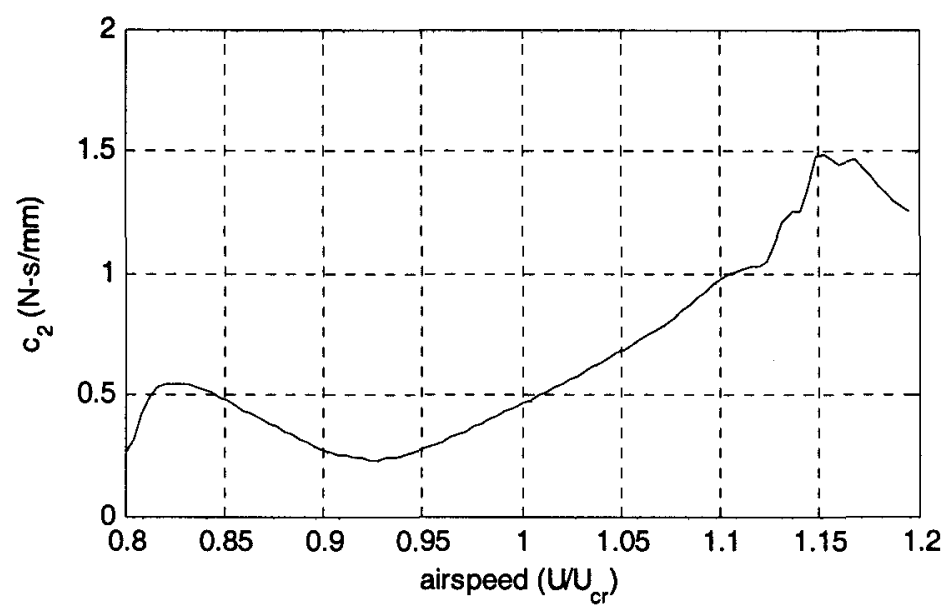

Fig. 7.14. The values of $c_{2}$ during flight simulation.

\subsubsection{Simulation with variable air density}

Assume air density $\rho$ changes as in Eq. (7.2) and $\rho_{0}=1.2, \mu=0.05,0.1 ; \omega=2 \pi$.

They are the same as the values in Chapter 7.1.3. The time response of the pitch-plunge airfoil under random external excitation is simulated with airspeed accelerating continuously from $U=0.8$ to $1.2 U_{\mathrm{cr}}$ at a linear rate for 30 seconds. The external excitation $f_{\mathrm{a}}$ with mean zero and standard deviation $1 \mathrm{kN}$ is applied over the 30 second time period. Results of tunable Maxwell DVA which is controlled based on scaled measured vibration spectra signals by the neural network model are shown in Fig. 7.15 and 7.16 where the translational displacement $w$ and the rotational movement $\alpha$ are stable at the cases of the airspeeds under $U=1.13$ and $1.11 U_{\mathrm{cr}}$. It is much better than the method which is based on the airspeed in the cases of flying with variable air density 

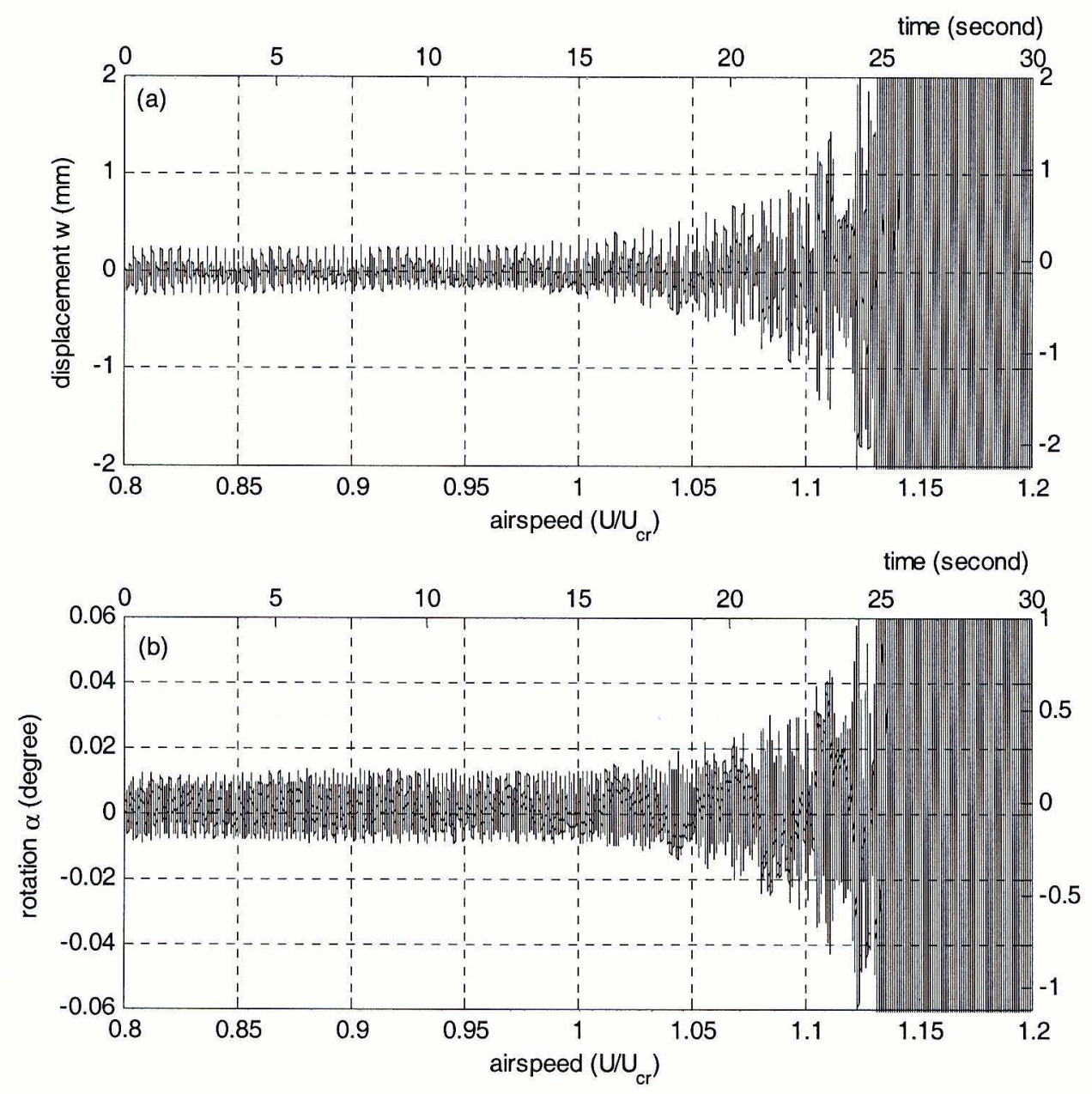

Fig. 7.15. Time responses of pitch-plunge airfoil with the neural-network based controller with $\mu=0.05$. 

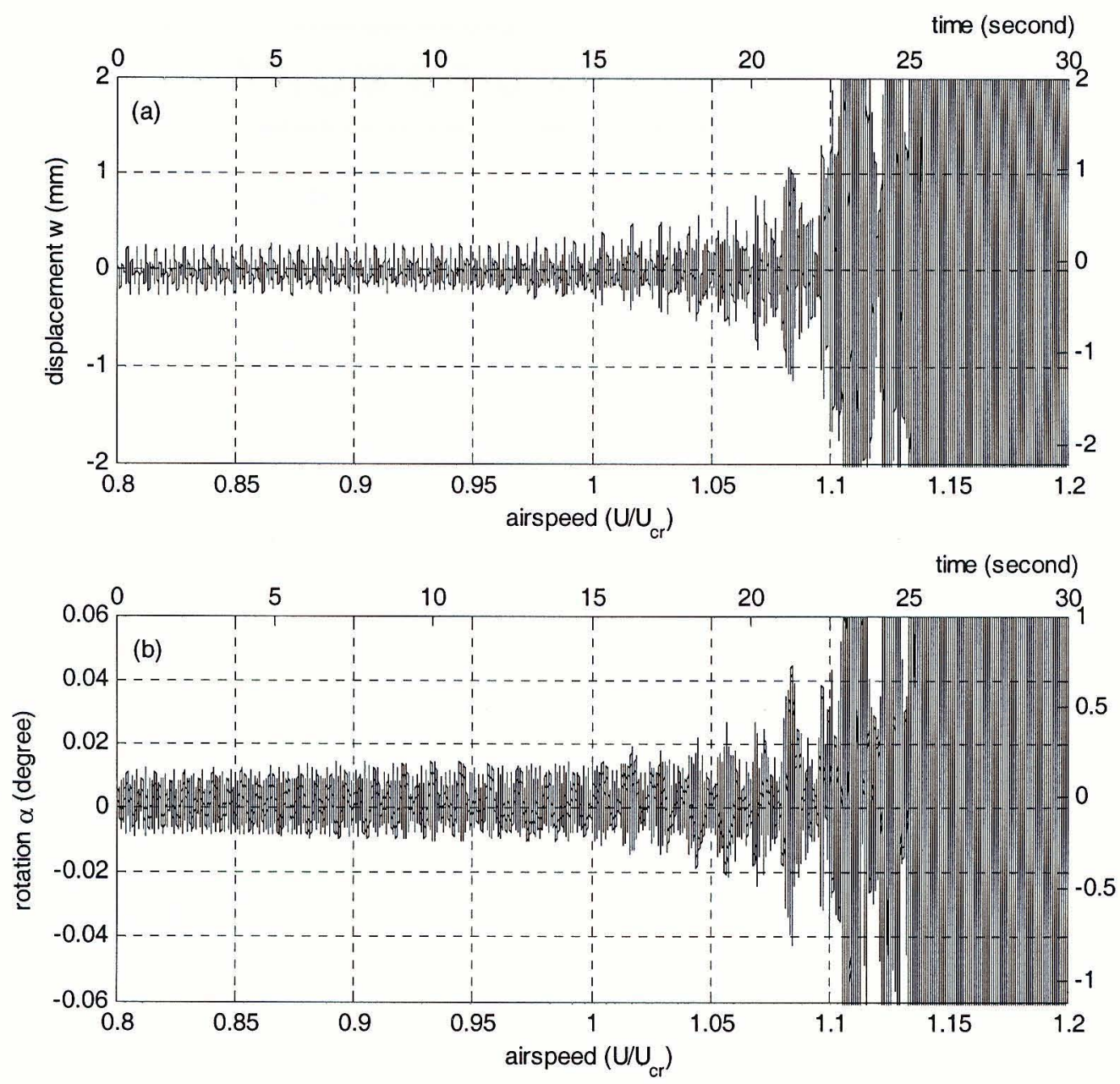

Fig. 7.16. Time responses of pitch-plunge airfoil with the neural-network based controller with $\mu=0.1$.

\subsection{Summary}

In this chapter, two control methods for the tunable Maxwell element DVA are investigated. First control method is based on the measured airspeed. It works well in the case of the air density is constant during flight. In the cases of the air density is variable during flight, a method based on the measured scaled vibration spectra is 
developed. Due to the complexity of the relationship between the measured vibration spectra and the optimized value of tunable damper, the neural network model is employed to establish the relationship. Both control methods are based on the measured signals and do not require prior knowledge of the plant parameters. 


\section{CHAPTER 8 \\ CONCLUSIONS, CONTRIBUTIONS AND FUTURE RESEARCH}

\subsection{Research on Elastomeic Isolators}

In this dissertation, modal analysis of a single mass elastomeric isolation system represented by a Maxwell-Voigt (M-V) model is conducted and the influences that the stiffness $k_{1}$ and damping $c_{1}$ of the Maxwell element have on the natural frequency and damping ratio of the $\mathrm{M}-\mathrm{V}$ model is revealed. Three types of Maxwell elements are defined and are distinct by their time constant, $\tau_{1}=c_{1} / k_{1}$. If $\tau_{1}$ is small, the Maxwell element is Type $A$ - damping dominant. If $\tau_{1}$ is large, the Maxwell element is Type $C$ stiffness dominant. Falling between these two element types is the Type B Maxwell element - neither damping nor stiffness dominant. The different influences that each of these types have on the system natural frequencies and damping ratio is discussed. It is also shown that the existence of the Maxwell element in the M-V model always results in a natural frequency greater than a Voigt model with equivalent Voigt elements as the M$\mathrm{V}$ model. However, this is not necessarily true for the damping ratio. For certain Maxwell element types, the damping ratio can actually be less than the Voigt model.

Harmonic analysis reveals that M-V models with unlike parameters can have the same natural frequency and damping ratio and different frequency response functions. Consequently, M-V models are more capable of representing elastomeric isolation 
systems over a broad frequency range. This is unlike Voigt models whose frequency response functions are fixed once their natural frequency, damping ratio and either mass or stiffness are determined. Consequently, a parameter identification method is developed. The method is based upon constant natural frequency and damping ratio curves of a M-V frequency response model. These curves are determined from the parametric data yielded from the modal analysis. In addition, it is shown that the frequency response function of a $\mathrm{M}-\mathrm{V}$ model exists between two limits. These limits are frequency response functions of two Voigt models, a mass consistent Voigt model (Voigt Model $M$ whose mass is equivalent to the mass of the M-V model) and a stiffness consistent Voigt model (Voigt Model $K$ whose stiffness is equivalent to the static stiffness of the M-V model). The effectiveness of the parameter identification method is verified by several analytical examples.

A parameter identification method based on constraint optimization is developed for general Maxwell models that have two or more Maxwell elements in a single mass isolation system by fitting the models to measured frequency response spectra. The effectiveness of the identification method is verified by several analytical examples. These studies reveal that a Maxwell model having only one Maxwell element can simulate the dynamic characteristics of a Maxwell system having two Maxwell elements as long as one is not Type $B$ and the other is not Type $C$. These analytical studies also conclude that a Maxwell model having two Maxwell elements (One Type B and one Type C) can simulate the dynamic characteristics of a Maxwell system having more than two Maxwell elements. 
Experiments on real commercial elastomeric isolators are conducted with three different rubber isolators subjected to both static and dynamic experiments. For all three rubber isolators, it is shown that although identified stiffness consistent Voigt models can match the static stiffness, natural frequency and damping ratio, they cannot match the measured frequency response. Meanwhile, identified mass consistent Voigt models match measured natural frequencies and damping ratios, although they cannot match the measured static stiffness and frequency response. Identified Maxwell models having only one Maxwell element can match the measured natural frequency, damping ratio and static stiffness, but cannot match the measured frequency response curves well. However, using the method developed in this study, identified Maxwell models having two Maxwell elements can accurately represent the measured static and dynamic characteristics of real elastomeric isolation systems.

The most important contributions of this:

1. Define three different types of Maxwell elements. Reveal the influences of the different types have on the dynamic properties of isolator models.

2. System identification method for $\mathrm{M}-\mathrm{V}$ model.

3. Illustrate that a model with two Maxwell elements can adequately represent general Maxwell systems having more than two Maxwell elements.

4. Developed system identification approach based on constraint optimization that was successfully employed for the identification of M-M-V model for three commercially available rubber mounts.

Future work in this area should concentrate on the research of nonlinear stiffness and damping behavior in case of large deflection condition. 


\subsection{Research on DVA Control of Aircraft Wing}

The dynamic analysis reveals that the dynamic properties of aeroelastic aircraft wing such as natural frequencies and damping ratios change as airspeed changes. The aeroelastic aircraft wing turns to be unstable at high airspeed condition. The DVA can improve the dynamic behavior at high airspeed condition. Further investigation reveals that the DVA with tunable stiffness and damping components performs better than the constant parameter DVA. Because the tunable stiffness component is not well developed yet, an alternative configuration of tunable Maxwell element DVA which has only one tunable damping component is designed. Dynamic analysis and simulation reveal the performance of this tunable Maxwell element DVA close to the tunable DVA which has both tunable stiffness and damping components.

Two control methods for the tunable Maxwell element DVA are investigated. First control method is based on the measured airspeed. It works well in the case the air density is constant during flight. In the cases of air density is variable, a method based on the measured scaled vibration spectra is developed. Due to the complexity of the relationship between the measured scaled vibration spectra and the optimized value of tunable damper, the neural network model is employed to establish the relationship. Both control methods are based on the measured signals and it does not require prior knowledge of the plant parameters.

The most important contributions of the work:

1. A tunable Maxwell element DVA was investigated for aeroelastic aircraft wing vibration and flutter suppression. 
2. The critical airspeed of aircraft wing has been increased more than $14 \%$ by using a tunable DVA.

3. Using a tunable Maxwell element DVA having has only one tunable damper replace the DVA which has both tunable stiffness and damping components and achieving similar vibration absorption effect.

4. A neural network is used to build a relationship between the measured vibration spectra and the optimal damping coefficient of a tunable Maxwell element DVA for vibration control.

Future work in this area should concentrate on the investigation in the approach of experiment. 


\section{REFERENCES}

[1] Flügge, W., 1975, Viscoelasticity, New York: Springer-Verlag; Second Revised Edition.

[2] Ravindra, B., and Mallik, A. K., 1994, "Performance of non-linear vibration isolators under harmonic excitation", Journal of Sound and Vibration, Vol. 170, pp. 325-337.

[3] Lee, J. H., and Kim, K. J., 2002, "Treatment of frequency-dependent complex stiffness for commercial multi-body dynamic analysis programs", Mechanics of structures and machines, Vol. 30(4), pp. 527-541.

[4] Dejong, R. G., Ermer, G. E., Paydenkar, C. S., and Remtema, T. M., 1998, "High frequency dynamic properties of rubber isolation elements", Proceedings of NoiseCon '98, pp. 383-390.

[5] D. H. Cooper, 1959, "Method of applying the results of dynamic testing to rubber anti-vibration systems", Transactions of the Institution of the Rubber Industry, Vol. 35, pp. 166-1770.

[6] Lin, T. R., Farag, N. H., and Pan, J., 2005, "Evaluation of frequency dependent rubber mount stiffness and damping by impact test", Applied Acoustics, Vol. 66, pp. 829-844. 
[7] Thompson, D. J., Van Vliet, W. J., and Verheij, J. W., 1998, "Developments of the indirect method for measuring the high frequency dynamic stiffness of resilient elements", Journal of Sound and Vibration, Vol. 213(1), pp. 169-188.

[8] Kim, S., and Singh, R., 2001, "Multi-dimensional characterization of vibration isolators over a wide range of frequencies", Journal of Sound and Vibration, Vol. 245, pp. 877-913.

[9] Cuvalci, O., Ertas, A., Ekwaro-Osire, S., and Cicek, I., 2002, "Non-linear vibration absorber for a system under sinusoidal and random excitation: experiments", Journal of Sound and Vibration, Vol. 249(4), pp. 701-718.

[10] Carneal, J. P., Charette, and F., Fuller, C. R., 2004, "Minimization of sound radiation from plate using adaptive tuned vibration absorbers", Journal of Sound and Vibration, Vol. 270, pp. 781-792.

[11] Webster, A., and Semke, W., 2004, "Frequency-dependent viscoelastic structural elements for passive broad-band vibration control", Journal of Vibration and Control, Vol. 10, pp. 881-895.

[12] Holdhusen, M. H., and Cunefare, K, A., 2003, "Damping effect on the stateswitched absorber used for vibration suppression", Journal of Intelligent Material Systems and Structures, Vol. 14, pp. 551-561.

[13] Richards, C. M., Brenner, M. J., and Singh, R., 2004, "Identification of a nonlinear aeroelastic aircraft wing model", $45^{\text {th }}$ AIAA/ASME/ASCE/AHS/ASC Structures, Structural Dynamics, and Materials Conference, Palm Springs, CA.

[14] Dickens, J. D., 2000, "Phase velocity of rubber element in vibration isolator under static load", Journal of Sound and Vibration, Vol. 234, pp. 21-42. 
[15] Dickens, J. D., 2000, "Dynamic model of vibration isolator under static load", Journal of Sound and Vibration, Vol. 236, pp. 323-337.

[16] Kari, L., 2003, "On the dynamic stiffness of preloaded vibration isolators in the audible frequency range: modeling and experiments", Journal of the Acoustical Society of America, Vol. 113(4), pp. 1909-1921.

[17] Li, W. L., and Lavrich, P., 1998, "Determination of power flows through vibration isolators", Proceedings of Noise-Con '98, pp. 377-382.

[18] Kim, S. and Singh, R., 2001, "Vibration transmission through an isolator modeled by continuous system theory", Journal of Sound and Vibration, Vol. 248, pp. 925-953.

[19] Wang, L. R., Lu, Z. H., and Hagiwara, I., 2002, "Finite element simulation of the static characteristics of a vehicle rubber mount", Proceedings of the institution of mechanical engineers, Part D: Journal of automobile engineering, Vol. 216(12), pp. 965-973.

[20] Chandra Shekhar, N., Hatwal, H., and Mallik, A. K., 1998, "Response of nonlinear dissipative shock isolators", Journal of Sound and Vibration, Vol. 214, pp. 589-603.

[21] Chandra Shekhar, N., Hatwal, H., and Mallik, A. K, 1999, "Performance of nonlinear isolators and absorbers to shock excitation", Journal of Sound and Vibration, Vol. 227, pp. 293-307.

[22] Kirk, C. L., 1988, "Non-linear random vibration isolators", Journal of Sound and Vibration, Vol. 124, pp. 157-182. 
[23] Adiguna, H., Tiwari, M., Singh, R., Tseng, H. E., and Hrovat, D., 2003, "Transient response of a hydraulic engine mount", Journal of Sound and Vibration, Vol. 268, pp. 217-248.

[24] Richards, C. M., and Singh, R., 1998, "Identification of nonlinear properties of rubber isolators using experimental and analytical methods", Proceedings of NoiseCon '98, pp. 391-396.

[25] Richards, C. M., and Singh, R., 2001, "Characterization of rubber isolator nonlinearities in the context of single- and multi-degree-of-freedom experimental systems", Journal of Sound and Vibration, Vol. 247(5), pp. 807-834.

[26] Ni, Y. Q., Ko, J. M., and Wong, C. W., 1998, "Identification of nonlinear hysteretic isolators from periodic vibration tests", Journal of sound and Vibration, Vol. 217(4), pp. 737-756

[27] Tan, R. Y., and Huang, M. C., 2000, "System identification of a bridge with lead-rubber bearings", Computer and Structures, Vol. 74, pp. 267-280

[28] Hong, W. K., and Kim, H. C., 2004, "Performance of a multi-story structure with a resilient-friction base isolation system", Computer and Structures, Vol. 82, pp. $2271-2283$

[29] du Flopy, N. F., Heyns, P. S., and Brennan, M. J., 2005, "The development of a tunable vibration absorbing isolator", International Journal of Mechanical Sciences, Vol. 47, pp. 983-997.

[30] Schmitendorf, W. E., 2000, "Designing tuned mass dampers via static output feedback: a numerical approach", Earthquake Engineering and Structural Dynamics, Vol. 29, pp. 127-137. 
[31] Zuo, L., and Nayfeh, S. A., 2006, "The two-degree-of freedom tuned-mass damper for suppression of single-mode vibration under random and harmonic excitation", ASME Journal of Vibration and Acoustics, Vol. 128, pp. 56-65.

[32] Davis, C. L., and Lesieutre, G. A, 2000, "An active tuned solid-state vibration absorber using capacitive shunting of piezoelectric stiffness", Journal of Sound and Vibration, Vol. 232(3), pp. 601-607.

[33] Rusovici, R., Dosch, J. J., and Lesieutre, G. A., 2002, "Design of a single-crystal piezoceramic vibration absorber", Journal of Intelligent Material Systems and Structures, Vol. 13, pp. 705-712.

[34] Jallili, N., and Knowles IV, D. W., 2004, "Structural vibration control using an active resonator absorber: modeling and control implementation", Smart Materials and Structures, Vol. 13, pp. 998-1005.

[35] Lee, J., Berger, E., and Kim, J. H., 2005, "Feasibility study of a tunable friction damper", Journal of Sound and Vibration, Vol. 283, pp. 707-722.

[36] Yong, C., Zimcik, D. G., Wickramasinghe, V. K., and Nitzsche, F., 2004, "Development of the smart spring for active vibration control of helicopter blades", Journal of Intelligent Material Systems and Structures, Vol. 15, pp. 37-47.

[37] Williams, K. A., Chiu, G. T. -C., and Bernhard, R. J, 2005, "Dynamic modeling of a shape memory alloy adaptive tuned vibration absorber", Journal of Sound and Vibration, Vol. 280, pp. 211-234.

[38] Hosek, M., and Olgac, N., 2002, “A single-step automatic tuning algorithm for the delayed resonator vibration absorber", IEEE/ASME Transactions on Mechatronics, Vol. 7(2), pp. 245-255. 
[39] Koo, J-H., Ahmadian, M., Setareh, and M., Murray, T. M., 2004, "In search of suitable control methods for semi-active tuned vibration absorbers", Journal of Vibration Control, Vol. 10, pp. 163-174.

[40] Koo, J-H., Ahmadian, M., and Setareh, M., 2006, "Experimental robustness analysis of magneto-rheological tuned vibration absorbers subject to mass offtuning", ASME Journal of Vibration and Acoustics, Vol. 128, pp. 126-131.

[41] Brayton, R. K., Director, S. W., Hachtel, G. D., and Vidigal, L., 1979, “A New Algorithm for Statistical Circuit Design Based on Quasi-Newton Methods and Function Splitting" IEEE Trans. Circuits and Systems, Vol. CAS-26, pp. 784-794.

[42] http://www.novibes.com/

[43] Meirovitch, L., 2001, Fundamentals of Vibration, Boston: McGraw-Hill.

[44] Hagan, M. T., Demuth, H. B., Beale, M. H., 2002, Neural Network Design, Boston: PWS.

[45] Chen, S., Cowan, C. F. N., and Grant, P. M., 1991, "Orthogonal least squares learning algorithm for radial basis function networks" IEEE Trans. Neural Networks, Vol. 2(2), pp. 302-309.

[46] Haykin, S., 2005, Neural Networks: A Comprehensive Foundation, New Delhi: Prentice-Hall, Second Edition, pp. 298-305.

[47] Fung, Y. C., 1955, An Introduction to the Theory of Aeroelasticity, New York: John Wiley \& Sons, pp. 192-195. 


\section{CURRICULUM VITAE}

NAME: $\quad$ Jie Zhang

ADDRESS: $\quad 778$ David Fairleigh Ct. \#4

Louisville, KY 40217

DOB: $\quad$ Qingdao, China - January 2, 1959

EDUCATION: B.S., Mechanical Engineering

\& TRAINING Zhejiang University, Hangzhou, China 1982

M.S., Mechanical Engineering

Zhejiang University, Hangzhou, China

1989

Ph.D. Mechanical Engineering

University of Louisville, Louisville, KY

2006

AWARDS: $\quad$ First class award by National Education Committee of China for the computer aided design and analysis of hydraulic excavator, 1994.

PROFESSIONAL

SOCIETIES: Vibration Engineering Society of China.

American Society of Mechanical Engineers.

PUBLICATIONS:

Zhang, Jie and Richards, Christopher M., "Dynamic analysis and parameter identification of a single mass elastomeric isolation system using a Maxwell-Voigt model", ASME Journal of Vibration and Acoustics, in press. 
Zhang, Jie and Richards, Christopher M., "Parameter identification of analytical and experimental rubber isolators represented by Maxwell models", Mechanical Systems and signal Processing, in review.

Zhang, Jie and Qi, Zhongwei, 2001.5, "The method and implement of mechanism design based on Intranet", China Mechanical Engineering, Vol. 12.

Zhang, Jie, 2000.6, "The development of fixture computer aided design system for Machine-Tools", Journal of Qingdao University, Vol. 13(2).

Zhang, Jie and Fang, Yi, 2000.2, "The computer aided design of the trade-mark-paper picking mechanism of beer machinery", Machinery, Vol. 27(1).

Zhang, Jie, 1999.9, "Wire cutting tools programming system based on the AutoCAD software", Journal of Qingdao University, Vol. 12(3).

Zhang, Jie, 1999.2, "The Application of Object-Oriented Programming Method in the Motion Simulation for the Trade-markpaper Picking Mechanism of Beer Machinery", Modern Machinery, Vol. 98.

Zhang, Jie, 1998.9, "The numerical description and generation algorithm for curves", Journal of Qingdao University, Vol. 11(3).

Zhang, Jie, 1996.6, "Research on dynamic modeling and parameter identification of joints between complicated mechanical structures", Journal of Mechanical Strength, Vol. 18(2).

Zhang, Jie and Tong, Zhongfang, 1994.9, "The problem of dynamic modeling of bolted joints in Machine-Tools", Journal of Vibration and Shock, Vol. 13(3).

Zhang, Jie and Tong, Zhongfang, 1993.9, "A method of building dynamic models with high precision and low dimension for the complex damping vibration systems", Journal of Vibration and Shock, Vol. 12(3). 
Zhang, Jie and Tong, Zhongfang, 1993.3, "Research on the mechanical dynamic parameter identification", Joumal of Qingdao University, Vol. 6(2).

Zhang, Jie and Feng, Peien, 1992.12, "External load calculation on nodes of crawler type hydraulic excavator for finite element analysis", Construction Machinery and Equipment, Vol. 23(12).

TEXTBOOK: Zhang, Jie and Shen, Jinghu, 2000.7, Engineering Design Methodology on Internet/Intranet, People's Posts \& Telecommunications Publishing House, Beijing, China. 